Aus der ehem. Abteilung Anaesthesiologie II

(Ehem. Leiter: Prof. Dr. med. H. Burchardi)

im Zentrum Anaesthesiologie, Rettungs - und Intensivmedizin

der Medizinischen Fakultät der Universität Göttingen

\title{
Direkte, patientenbezogene Kosten der intensivmedizinischen Behandlung in Abhängigkeit des Krankheitsschwerescores SAPS II und des Pflegeaufwandscores TISS - 28
}

\author{
INAUGURAL - DISSERTATION \\ zur Erlangung des Doktorgrades \\ der Medizinischen Fakultät der \\ Georg - August - Universität zu Göttingen \\ vorgelegt von \\ Sandy Büscher (geb. Rennecke) \\ aus Staßfurt
}


Dekan:

I. Berichterstatter:

II. Berichterstatter/in:

III. Berichterstatter/in:
Prof. Dr. med. C. Frömmel

Prof. Dr. med. H. Burchardi

Priv.- Doz. Dr. med. Schmidt

Tag der mündlichen Prüfung: 14. März 2012 
2.

Material und Methoden

2.1. Die Scores SAPS II und TISS - 28 6

2.1.1. Der SAPS II - Score 6

2.1.2. Der TISS - 28 - Score

2.2. Das Patientenkollektiv und die Datenerfassung aus der Patientenakte in dieser Studie 12

2.3. Die Software zur Datenerfassung in dieser Studie 13

2.3.1. Die Datenerfassung der verbrauchten Ressourcen und erbrachten Leistungen in den einzelnen Masken des DIVI - COSt - Computerprogramms 14

2.3.1.1. Medikamente 14

2.3.1.2. Perfusortherapie 15

2.3.1.3. Blutprodukte und Gerinnungsfaktoren 16

2.3.1.4. Volumenersatz und Basisinfusionen 17

2.3.1.5. Ernährung und Supplemente 18

2.3.1.6. Beatmung 19

2.3.1.7. Invasive Maßnahmen / Monitoring 20

2.3.1.8. Sonstige Maßnahmen / Diagnostik 21

2.3.1.9. Laboranalysen 22

2.3.1.10. Mikrobiologie 23

2.3.2. Die Dokumentation der Scores SAPS II und TISS - 28

2.3.3. Personal - und Overheadkosten 26

2.4. Die Berechnung der verursachten Kosten 27

2.5. Die Einteilung der Patienten in LOS - Gruppen und die Auswahl der Musterpatienten A - D 28

2.6. Die statistische Vorgehensweise 28 
3

Ergebnisse

3.1. Deskriptive Statistik des Patientenkollektives 29

3.1.1. Die Verteilung der Diagnosen im Patientenkollektiv 29

3.1.2. Die Verteilung des Patientenalters 30

3.1.3. Die Verteilung der Patientenliegedauer 31

3.1.4. Die Verteilung der Patientengesamtkosten 32

3.1.4.1. Die Verteilung der Diagnostik -, Labor -, Medikamenten -, Overhead - und Personalkosten als Anteil der Gesamtkosten 33

3.2. Die Korrelationen zwischen Scores und Kosten 36

3.2.1. Die Korrelationen zwischen Gesamtscores und Gesamtkosten 36

3.2.1.1. Die Korrelation zwischen dem Gesamt - SAPS II und den Gesamtkosten 36

3.2.1.2. Die Korrelation zwischen dem Gesamt - TISS - 28 und den Gesamtkosten

3.2.1.3. Die Korrelation zwischen dem Gesamt - SAPS II bzw. dem Gesamt - TISS -28 und den gesamten Diagnostik -, Labor - und Medikamentenkosten als Anteil der Gesamtkosten

3.2.2. Die Korrelation zwischen der Patientenliegedauer (LOS) und den Gesamtkosten

3.2.3. Die Korrelation zwischen den Gesamtscorewerten und den Gesamtkosten in einzelnen LOS - Patientengruppen

3.2.3.1. Die Korrelation zwischen dem Gesamt - SAPS II und den Gesamtkosten bei Patienten mit einer LOS unter 5 Tagen

3.2.3.2. Die Korrelation zwischen dem Gesamt - SAPS II und den Gesamtkosten bei Patienten mit einer LOS von 5 - 10 Tagen

3.2.3.3. Die Korrelation zwischen dem Gesamt - SAPS II und den Gesamtkosten bei Patienten mit einer LOS von über 10 Tagen 
3.2.3.4. Die Korrelation zwischen dem Gesamt - TISS - 28 und den Gesamtkosten bei Patienten mit einer LOS unter 5 Tagen

3.2.3.5. Die Korrelation zwischen dem Gesamt - TISS - 28 und den Gesamtkosten bei Patienten mit einer LOS von 5 - 10 Tagen

3.2.3.6. Die Korrelation zwischen dem Gesamt - TISS - 28 und den Gesamtkosten bei Patienten mit einer LOS von über 10 Tagen

3.3. Die Verteilung der Personal -, Diagnostik -, Labor - und Medikamentenkosten bei einzeln ausgewählten Musterpatienten

3.3.1. Die Verteilung der Personal -, Diagnostik -, Labor - und Medikamentenkosten zweier Musterpatienten ( $\boldsymbol{A}$ und $\boldsymbol{B}$ ) mit einer LOS unter 5 Tagen

3.3.2. Die Verteilung der Personal -, Diagnostik -, Labor - und Medikamentenkosten eines Musterpatienten $\boldsymbol{C}$ mit einer LOS von 5 - 10 Tagen

3.3.3. Die Verteilung der Personal -, Diagnostik -, Labor - und Medikamentenkosten eines Musterpatienten $\boldsymbol{D}$ mit einer LOS von über 10 Tagen

4.

$$
\text { Diskussion }
$$

4.1. Die Einführung des DRG - Systems 56

4.2. Die Kostenerfassung 58

4.3. Kostenvergleiche 62

4.4. Der Einfluss der Patientenliegedauer 64

4.5. Die Personalkosten 68

4.6. Die Diagnose - bezogenen Kosten 69

4.7. Verfahrenskritik 73

4.8. Die Entwicklung eines kostengerechten Aufwandmarkers 74

4.9. Ausblick 81 
5. Zusammenfassung

6. Abkürzungsverzeichnis

7. Literaturverzeichnis 


\section{Einleitung}

Die immensen Fortschritte der modernen Medizin wären ohne die Intensivmedizin nicht denkbar. So konnte in der Vergangenheit ein großer Entwicklungsfortschritt in der intensivmedizinischen Patientenversorgung durch einen hohen Aufwand in den Bereichen der Forschung sowie der Weiterentwicklung der apparativen Diagnostik und Pharmakotherapie erreicht werden. Daher verbraucht die Intensivmedizin in der Regel einen beträchtlichen Teil der Gesamtausgaben eines Krankenhauses. Es ist also ein Kostenbereich, der große Aufmerksamkeit verdient. Nach Angaben der Deutschen Krankenhausgesellschaft wurden 1999 auf deutschen Intensivstationen 6.226.000 Patiententage verbracht. Dabei entstanden Kosten in einer Höhe von 5,3 Millionen Euro (Moerer et al. 2002).

Die durchschnittlichen täglichen Patientenkosten betragen auf internistischen Intensivstationen in Deutschland 1.336 Euro (Graf et al. 2002), in Großbritannien zwischen 1.396 und 1.647 Euro (Edbrooke et al. 1999) und in Kanada 1.007 Euro (Noseworthy et al. 1996). Auf interdisziplinären Intensivstationen liegen die täglichen Kosten pro Patient bei 1.715 Euro in Italien (lapichino et al. 2004) und bei 951 Euro in Kanada (Heyland et al. 1998). Die Behandlung allgemeinchirurgischer Intensivpatienten kostet in Deutschland durchschnittlich 1.050 Euro pro Tag (Billing et al. 2004). Neurochirurgische Intensivpatienten verursachen in Neuseeland sogar durchschnittliche Tageskosten von 2.051 Euro (Havill et al. 1998).

An diesen Kosten wird deutlich, welche beträchtlichen finanziellen Anforderungen an die Gesundheitssysteme in hoch zivilisierten Ländern durch die intensivmedizinische Patientenversorgung resultieren. Diese Problematik wurde in Deutschland auch seitens des Gesetzgebers erkannt, welcher darauf mit der Einführung eines Fallpauschalen Vergütungssystems (Diagnosis - Related - Group (DRG) - System) reagierte. Dabei diente das australische Patientenklassifikationssystem (AR - DRG) als Grundlage für die deutsche Klassifikation von Fallpauschalen. Seit der Umstellung der Krankenhausfinanzierung auf pauschalierte Entgelte ist insbesondere die Vergütung der 
Intensivbehandlung problematisch geworden, da die gesamte Behandlung eines Patienten über einen Festpreis vergütet wird und somit vom individuellen Krankheitsverlauf, vom therapeutischen Aufwand und der Liegedauer unabhängig ist. Mehrere Studien zeigen jedoch, dass die intensivmedizinischen Kosten in erster Linie abhängig von der Liegedauer des Patienten sind (Heyland et al. 1998, Moerer und Burchardi 2001, Staudacher et al. 2003, Neilson et al. 2004). Nicht vorhersehbare Komplikationen, wie beispielsweise eine Beatmung, Infektion oder Sepsis, welche die intensivstationäre Liegezeit deutlich verlängern können, machen daher die Vergütung der Intensivmedizin in deutschen Krankenhäusern durch pauschalierte Entgelte zusätzlich schwierig. Dies betrifft vor allem Krankenhäuser der Maximalversorgung, da hier die schwer erkrankten und aufwändig zu behandelnden Patienten innerhalb einer DRG - Klassifikation versorgt werden müssen (Schmidt et al. 2004). Die Kosten der Behandlung von schwerstkranken Intensivpatienten im Rahmen der Maximalversorgung liegen über den Durchschnittskosten und unterscheiden sich eklatant von denen der Grund - und Regelversorgung (Moerer et al. 2007). Die Vergütung durch eine Einheitspauschale würde der Situation der Maximalversorgung keinesfalls gerecht (Billing et al. 2004).

Ein qualitativ gutes Gesundheitssystem ist hochdifferenziert und zeichnet sich durch eine dem Krankheitsbild und -schweregrad eines Patienten angemessene Versorgung in entsprechend ausgelegten Einrichtungen aus. Die Vergütung erfolgt jedoch nach Durchschnittswerten („Fallpauschalen“). Da hier aber besonders aufwändige Behandlungsfälle kaum sachgerecht vergütet werden können, wirft das DRG - Prinzip erhebliche Probleme auf. So ist ein grundsätzlicher Mangel des derzeitigen Fallpauschalensystems, dass bei der standardisierten Kalkulation der Vergütungswerte weder Art noch Qualität der Versorgung hinterfragt werden (Burchardi 2004). Über die Effizienz einer intensivmedizinischen Behandlung entscheidet zukünttig neben der erzielten Überlebensrate auch der hierfür erbrachte Kostenaufwand. Eine fachkompetente Analyse des $G$ - DRG - Systems ist daher für dessen sinnvolle Weiterentwicklung essentiell (Billing et al. 2004). 
Über die realen Kosten der deutschen Intensivmedizin gab es bisher wenig individuelle und fallbasierte Daten. Aus diesem Grund wurden jüngsthin bundesweit mehrere Studien zur genauen Kostenerfassung und hinsichtlich der Möglichkeit der Kostensenkung auf den Intensivstationen durchgeführt. Eines dieser Projekte war die von der Deutschen Interdisziplinären Vereinigung für Intensivmedizin und Notfallmedizin (DIVI) und dem Verband der Universitätsklinika Deutschlands (VUD) gemeinsam durchgeführte „VUD - Kostenstudie“, eine Multicenterstudie an 9 Universitätskliniken mit insgesamt 17 Intensivstationen, deren Bestandteil auch die vorliegende Arbeit war. Diese beschäftigte sich mit den direkten, patientenbezogenen Kosten. Dabei wurden sämtliche verbrauchten Ressourcen und erbrachten Leistungen dokumentiert und die daraus resultierenden Kosten der Intensivbehandlung errechnet. Die Analyse der Kosten erfolgte nach der aufwändigen Bottom - up - Methode. Auch wenn es verlockend erscheint, für diesen Zweck schnellere Methoden anzuwenden, oder sogar Rückrechnungen aus erhaltenen Krankenkassenzahlungen zu erwägen, müssen für eine valide, realistische Kostenanalyse die täglichen direkten, patientenbezogenen Kosten mühsam dokumentiert werden. Um Kostenersparnis erreichen zu können, ist es wichtig, die tatsächlichen Kostenfaktoren im Detail zu identifizieren (Noseworthy et al. 1996).

Das Ziel der vorliegenden Studie war es, die Kosten pro DRG- Fall zu identifizieren, und somit alternative Vergütungskonzepte für die Intensivmedizin - unter Berücksichtigung des individuellen Krankheits und Therapieverlaufs eines Patienten - zu finden. Dabei sollte im Rahmen der VUD - Kostenstudie geprüft werden, ob es möglich ist, die intensivmedizinischen Patientenkosten anhand des Krankheits schwerescores SAPS (Simplified Acute Physiology Score) und des Pflegeaufwandscores TISS (Therapeutic Intervention Scoring System) zu schätzen, so dass sich eine Vergütung der intensivmedizinischen Patientenkosten gegebenenfalls nach diesen Scores richten könnte. 
Seit dem Beginn der Entwicklung von modernen Scoringsystemen, ausgehend von Virginia Apgar im Jahr 1953, hat es eine Reihe von neuen Scoringsystemen gegeben, welche sich nunmehr in der dritten bzw. vierten Generation befinden. Eine Vielzahl von Scoringsystemen ist für den Bereich der Intensivmedizin entwickelt worden (Brenck et al. 2008). Man unterscheidet dabei Aufnahme- und Verlaufscores. Ferner können die täglich zu erfassenden Instrumente, je nach Zielkriterium, in weitere Gruppen kategorisiert werden: objektive Beschreibung des Grades der Organdysfunktion, Verlaufsbeschreibung während der Intensivtherapie, Einschätzung des Pflegeaufwandes, Bestimmung des Outcomes bzw. Mortalitätsrisikos, Gruppierung von Patientenkollektiven für Studienzwecke, Qualitätsmanagement, Kosten - Nutzen - Analysen und Hilfe bei der ärztlichen Entscheidungsfindung.

Bereits seit dem Jahr 1981 gibt es Vorschläge, den Krankheitsschweregrad eines intensivmedizinischen Patienten mit Zahlen zu bewerten. Hierzu wurde zunächst der sog. APACHE - Score (Acute Physiology And Chronic Health Evaluation) entwickelt (Knaus et al. 1985). Neben dem APACHE wird heute zur Evaluation der Krankheitsschwere der auch in dieser Arbeit verwendete SAPS - Score eingesetzt. Sowohl dem Scoremodell SAPS, als auch dem APACHE liegt die Validierung verschiedener Organsysteme zugrunde, wobei stets die am Meisten vom physiologischen Normalzustand abweichende pathologische Dysfunktion innerhalb der letzten 24 erfassten Stunden als Zahlenwert in die Gesamtbewertung einfließt (Le Gall et al. 1993). Die Summe der Zahlenwerte wird schließlich als Punktzahl angegeben und liegt umso höher, je schwerer der Krankheitszustand des Patienten ist. Der SAPS ist in Europa und Nordamerika erprobt und findet hier auch in der Praxis Verwendung, da er statistisch gewichtet ist und einen linearen Anstieg aufweist.

Auch ist es bezüglich der Kosteneffizienz in zunehmendem Maße wichtig, die Abläufe auf Intensivstationen zu beurteilen. Der TISS - Score, welcher 1974 von Cullen et al. entwickelt wurde, war hierbei ein großer Durchbruch. Obwohl er anfangs entwickelt wurde, um das Ausmaß der Krankheit zu erfassen, bekam er später größere Bedeutung, um 
Ressourcen zu messen (Moreno und Morais 1997). Heute dient der TISS der Beurteilung des Pflegeaufwandes bei Intensivpatienten und setzt sich aus der Auflistung mehrerer Pflegeaktivitäten zusammen, denen verschiedene Punktwerte zugeordnet werden. Mit Zunahme des Pflegeaufwandes für den einzelnen Patienten steigt dessen Wert des Gesamtscores. Der TISS hat sich als zuverlässiger Indikator pflegerischen Arbeitsaufwandes erwiesen (Cullen et al. 1974).

Scoresysteme stellen, gerade in Zeiten des Kostendrucks in der Intensivmedizin, eine Möglichkeit dar, das Patientenkollektiv zu beschreiben, aus der Behandlung entstehende Kosten transparent zu machen, sowie die Effizienz der Therapie darzustellen und vergleichend zu evaluieren. Es ist daher sinnvoll, in der vorliegenden Studie auch die prinzipielle Eignung der Scores SAPS II und TISS - 28 in dieser Hinsicht einer Überprüfung zu unterziehen.

\section{Fragestellung}

Ziel der vorliegenden Arbeit ist es, die direkten, patientenbezogenen Kosten der internistischen Intensivstation 1026 des Universitätsklinikums Göttingen zu erfassen und diese mit dem Krankheitsschwerescore SAPS II sowie dem Pflegeaufwandsscore TISS - 28 zu vergleichen. Dabei soll geprüft werden, inwiefern die intensivmedizinischen Patientenkosten mit den Scores korrelieren und innen dadurch nicht nur eine medizinische, sondern auch ökonomische Aussagekraft über den individuellen Krankheitsverlauf zukommt. Von übergeordnetem Interesse ist dabei, ob eine an diesen Scores orientierte Vergütung möglich ist. Anhand der Ergebnisse dieser Untersuchung sollen objektive Kriterien der intensivmedizinischen Behandlung entwickelt werden, welche eine sachlich begründete Leistungsvergütung für die Intensivmedizin ermöglichen. 


\section{Material und Methoden}

\subsection{Die Scores SAPS II und TISS - 28}

\subsubsection{Der SAPS II - Score}

Seit 1981 wurden für Intensivstationen verschiedene Scores zur Validierung der Krankheitsschwere vorgeschlagen. Die ersten (APACHE und SAPS) waren eine eher subjektive Methode zur Einschätzung der Mortalität. Um einen zuverlässigen und praktikablen Score zu entwickeln, wurde von September 1991 bis Februar 1992 in 12 Ländern eine Europäische/ Nordamerikanische Multicenterstudie durchgeführt. Das Ziel war es, einen allein physiologischen Score zur Krankheitsschwereabschätzung zu entwickeln. Durch die große Anzahl der in dieser Studie eingeschlossenen internistischen und chirurgischen Patienten konnte somit der Simplified Acute Physiology Score II (SAPS II), bestehend aus 17 zuverlässigen Variablen zur Einschätzung der Mortalität, ohne eine spezifische Diagnose zu kennen, entstehen. Das war der Anfang, um in Zukunft die Effizienz der Intensivstationen zu evaluieren. Jedoch ist zu berücksichtigen, dass das System aus einer großen, heterogenen Datenmenge entwickelt wurde und somit nur die Prognose eines Durchschnittspatienten bzw. einer vergleichbaren Patientengruppe berücksichtigt. Eine Prognose für den individuellen Patienten ist nicht zulässig (Le Gall et al. 1993).

Sowohl dem Scoremodell des SAPS II als auch dem APACHE II liegt die Validierung verschiedener Organsysteme zu Grunde, wobei stets die am Meisten vom physiologischen Normalzustand abweichende pathologische Dysfunktion innerhalb der letzten 24 erfassten Stunden als Zahlenwert in die Gesamtbewertung einfließt (Le Gall et al. 1993). Die Summe der Zahlenwerte wird schließlich als Punktzahl angegeben und liegt umso höher, je schwerer der Krankheitszustand des Patienten ist. Die zu vergebenden Zahlenwerte bei der Evaluierung des SAPS II setzen sich wie folgt zusammen: 


\begin{tabular}{|c|c|c|c|c|c|c|c|c|c|c|}
\hline \multirow[b]{2}{*}{ Alter (Jahre) } & \multicolumn{4}{|c|}{ Punkte bei abnormal niedrigen Werten } & \multirow{2}{*}{$\begin{array}{c}\text { normal } \\
<40\end{array}$} & \multicolumn{5}{|c|}{ Punkte bei abnormal hohen Werten } \\
\hline & & & & & & $\begin{array}{c}40-59 \\
7\end{array}$ & $\begin{array}{c}60-69 \\
12 \\
\end{array}$ & $\begin{array}{c}70-74 \\
15\end{array}$ & $\begin{array}{c}75-79 \\
16\end{array}$ & $\begin{array}{l}>80 \\
18\end{array}$ \\
\hline $\begin{array}{l}\text { Herzfrequenz (pro } \\
\text { min.) }\end{array}$ & & & $\begin{array}{l}<40 \\
\mathbf{1 1} \\
\end{array}$ & $\begin{array}{c}40-69 \\
\mathbf{2} \\
\end{array}$ & & $\begin{array}{c}120-159 \\
\mathbf{4} \\
\end{array}$ & $\begin{array}{c}\geq 160 \\
7\end{array}$ & & & \\
\hline $\begin{array}{l}\text { Blutdruck (syst., } \\
\mathrm{mmHg} \text { ) }\end{array}$ & & & $\begin{array}{l}<70 \\
13\end{array}$ & $\begin{array}{c}70-99 \\
\mathbf{5}\end{array}$ & $100-199$ & $\begin{array}{c}\geq 200 \\
\mathbf{2}\end{array}$ & & & & \\
\hline Temperatur $\left({ }^{\circ} \mathrm{C}\right)$ & & & & & $<39,0$ & $\begin{array}{l}\geq 39,0 \\
\mathbf{3}\end{array}$ & & & & \\
\hline $\begin{array}{l}\text { Nur bei Beatmung oder } \\
\text { Pulmonalis-Katheter } \\
\mathbf{P a O}_{2}(\mathrm{mmHG}) / \mathbf{F i O} \mathbf{2}_{\mathbf{2}}\end{array}$ & & $\begin{array}{c}<100 \\
\mathbf{1 1}\end{array}$ & $\begin{array}{c}100-199 \\
\mathbf{9}\end{array}$ & $\begin{array}{c}>200 \\
6\end{array}$ & - & & & & & \\
\hline $\begin{array}{l}\text { Urinausscheidung } \\
(1 / \mathrm{Tag})\end{array}$ & & & $\begin{array}{c}<0,5 \\
\mathbf{1 1}\end{array}$ & $\begin{array}{c}0,5-0,99 \\
\mathbf{4}\end{array}$ & $\geq 1,0$ & & & & & \\
\hline $\begin{array}{l}\text { Harnstoff }(\mathrm{mg} / \mathrm{dl}) \\
\text { order }\end{array}$ & & & & & $<60$ & $60-179$ & $\geq 180$ & & & \\
\hline $\begin{array}{l}\text { Harnstoff-Stickstoff } \\
(\mathrm{mg} / \mathrm{dl})\end{array}$ & & & & & $<28$ & $\begin{array}{c}28-83 \\
6\end{array}$ & $\begin{array}{c}\geq 84 \\
10\end{array}$ & & & \\
\hline $\begin{array}{l}\text { Leukozyten } \\
\left(10^{3} / \mathrm{mm}^{3}\right)\end{array}$ & & & & $\begin{array}{l}<1,0 \\
\mathbf{1 2}\end{array}$ & $1,0-19,9$ & $\begin{array}{l}\geq 20 \\
\mathbf{3}\end{array}$ & & & & \\
\hline Kalium (mmol/1) & & & & $\begin{array}{c}<3 \\
\mathbf{3} \\
\end{array}$ & $3,0-4,9$ & $\begin{array}{c}\geq 5,0 \\
\mathbf{3}\end{array}$ & & & & \\
\hline Natrium $(\mathrm{mmol} / \mathrm{l})$ & & & & $\begin{array}{c}<125 \\
\mathbf{5}\end{array}$ & $125-144$ & $\begin{array}{c}\geq 145 \\
\mathbf{1}\end{array}$ & & & & \\
\hline $\begin{array}{l}\text { Serum Bikarbonat } \\
(\mathrm{mmol} / \mathrm{l})\end{array}$ & & & $\begin{array}{c}<15 \\
6\end{array}$ & $\begin{array}{c}15-19 \\
\mathbf{3}\end{array}$ & $\geq 20$ & & & & & \\
\hline Bilirubin (mg/dl) & & & & & $<4,0$ & $\begin{array}{c}4,0-5,9 \\
\mathbf{4}\end{array}$ & $\begin{array}{c}\geq 6,0 \\
\mathbf{9}\end{array}$ & & & \\
\hline $\begin{array}{l}\text { Glasgow Coma Scale } \\
\text { (vor Sedierung) }\end{array}$ & $\begin{array}{l}<6 \\
26\end{array}$ & $\begin{array}{l}6-8 \\
13\end{array}$ & $\begin{array}{c}9-10 \\
7\end{array}$ & $\begin{array}{c}11-13 \\
5\end{array}$ & $14-15$ & & & & & \\
\hline Vorerkrankungen & & & & & - & Metas & t. Ca. & hämat & Erkr. & $\begin{array}{c}\text { AIDS } \\
\mathbf{1 7}\end{array}$ \\
\hline Zuweisung auf ICU & & & & & $\begin{array}{l}\text { elektiv } \\
\text { chirur- } \\
\text { gisch }\end{array}$ & Medizin & h (ohne & un & $\begin{array}{l}\text { lant } \\
\text { isch }\end{array}$ & \\
\hline
\end{tabular}

Tabelle 1: Die Zusammensetzung des SAPS II - Scores aus den einzelnen Parametern (nach Le Gall et al. 1993, S. 2958).

Liegt die Herzfrequenz außerhalb des im SAPS II definierten Normbereichs von 70 - 119 pro Minute, werden stufenweise bis zu 11 Punkte vergeben, für den systolischen Blutdruck außerhalb von $100-199 \mathrm{mmHg}$ bis zu 13 Punkte und für die Körpertemperatur bei über $39^{\circ} \mathrm{C}$ bis zu 3 Punkte. Liegt die Urinausscheidung des Patienten unter 1,0 Liter pro Tag, werden stufenweise bis zu 11 Punkte hinzugerechnet. In Bezug auf die Harnstoff - Stickstoff - Konzentration im Blut vergibt man bei über $28 \mathrm{mg} / \mathrm{dl}$ bis zu 10 Punkte. Wahlweise kann hier auch, je nach im Labor üblicherweise bestimmtem Wert, die Harnstoffkonzentration im Blut 
in $\mathrm{mg} / \mathrm{dl}$ in den Score einfließen. Bei der Anzahl der Leukozyten werden außerhalb von 1,0 - 19,9 × 103/ $\mathrm{mm}^{3}$ maximal 12 Punkte addiert. Je nach Ausprägung der Entgleisung werden für die Elektrolyte Kalium (2,0 - 4,9 $\mathrm{mmol} / \mathrm{l})$ und Natrium (125 - $144 \mathrm{mmol} / \mathrm{l})$ bis zu 3 bzw. 5 Punkte vergeben sowie für das Serum Bikarbonat $(>20 \mathrm{mmol} / \mathrm{l})$ und Bilirubin $(<4 \mathrm{mmol} / \mathrm{l})$ maximal weitere 6 bzw. 9 Punkte addiert. Des Weiteren werden das Alter bei über 40 Jahren stufenweise mit bis zu 18 Punkten, die vor Sedierung erhobene Glasgow Coma Scale (normal 14 - 15) mit bis zu 26 Punkten, Vorerkrankungen in Form von metastasierenden Karzinomen, malignen hämatologischen Erkrankungen und AIDS mit insgesamt bis zu 36 Punkten und die Art der Zuweisung auf die Intensivstation, beispielsweise medizinisch geplant oder elektiv chirurgisch, mit bis zu 8 Punkten berücksichtigt. Bei beatmeten Patienten oder jenen mit Pulmonalis - Katheter kommen schließlich noch bis zu 11 mögliche Punkte für den Quotienten aus dem Sauerstoffpartialdruck in $\mathrm{mmHg}$ und dem fraktionellen Sauerstoffanteil in der Beatmungsluft in Prozent hinzu.

Die Daten des SAPS II zu erheben, ist sehr einfach und schnell möglich, da alle Variablen leicht verfügbar sind und keine speziellen diagnostischen Prozeduren erfordern. Diese einfache Benutzung und das hohe Interesse an der Mortalitätswahrscheinlichkeit spiegeln sich in der weit verbreiteten Akzeptanz des Scores wider (Le Gall et al. 1993). So zeigt der Score auch eine große Zuverlässigkeit in der Voraussage der Letalität (Schuster et al. 1996).

Ferner sind die einzelnen Parameter des SAPS II statistisch gewichtet, wurden in früheren Studien geprüft und überarbeitet. Die zweite Version des Scores, der sog. SAPS II, ist mittlerweile anerkannt als Maßstab für die Quantifizierung der Krankheitsschwere (Apolone et al. 1996; Metnitz et al. 1999; Vosylius et al. 2004). Auch in der vorliegenden Studie findet der SAPS II Verwendung.

Mittlerweile gibt es bereits eine Weiterentwicklung des Prognosesystems (SAPS III), die jedoch für die vorliegende Studie nicht berücksichtigt wurde (Metnitz et al. 2005, Moreno et al. 2005). 
In der vorliegenden Studie wurde die Korrelation zwischen den Tageskosten pro Patient und den Scorewerten ermittelt. Um unter anderem auch die Korrelation zwischen den gesamten Patientenkosten und den gesamten Scorewerten zu bestimmen, wurden die einzelnen Tageswerte der Scores über die gesamte Liegedauer (LOS) eines einzelnen Patienten zu einem Gesamtscore addiert, obwohl es für die klinische Anwendung des Scores nicht vorgesehen ist. Dies wurde sowohl für den SAPS II zum Gesamt - SAPS II, als auch für den TISS - 28 zum Gesamt - TISS - 28 vorgenommen.

\subsubsection{Der TISS - 28 - Score}

Schwerpunkt der Entwicklung des Therapeutic Intervention Scoring System (TISS) war es ursprünglich, eine Korrelation von TISS- Punkten einerseits und Schwere der pathophysiologischen Veränderungen andererseits herzustellen (Cullen et al. 1974). Heute dient der TISS aber schwerpunktmäßig dazu, die Höhe der Punktzahl in einen Zusammenhang mit dem Therapieaufwand, insbesondere dem zeitlichen Pflegeaufwand, und damit indirekt mit dem Personenbedarf von Intensivstationen zu bringen.

Zunächst bestand der TISS aus 76 einzelnen Parametern und wurde daher als TISS - 76 bezeichnet. Es konnten jedoch 28 Parameter gefunden werden, welche die gleiche Aussagekraft haben wie die ursprünglichen 76. Der Score wird seither als TISS - 28 bezeichnet und in dieser Form eingesetzt (Miranda et al. 1996).

Der TISS - Score dient der Beurteilung des Pflegeaufwandes bei Intensivpatienten und setzt sich aus der Auflistung mehrerer Pflegeaktivitäten zusammen, denen verschiedene Punktwerte zugeordnet werden. Mit Zunahme des Pflegeaufwandes für den einzelnen Patienten steigt dessen Wert des Gesamtscores. Der TISS hat sich als zuverlässiger Indikator pflegerischen Arbeitsaufwandes erwiesen (Cullen et al. 1974). 


\begin{tabular}{|c|c|c|}
\hline Basis & & Punkte \\
\hline $\begin{array}{l}\text { Standard- } \\
\text { Monitoring }\end{array}$ & $\begin{array}{l}\text { - stündliche Vitalzeichenkontrolle und } \\
\text { - regelmäßige Berechnung der Flüssigkeitsbilanz }\end{array}$ & 5 \\
\hline Labor & $\begin{array}{l}\text { - biochemische Bestimmungen und/oder } \\
\text { - Mikrobiologie (Blutkultur) am Tag der Abnahme }\end{array}$ & 1 \\
\hline Medikation & $\begin{array}{l}\text { - jedes Medikament; jede Dosierung } \\
\text { - jede Applikationsform (i.v., i.m., subkutan, Magensonde, oral) } \\
\text { - z. B. Sedierung, Antibiotika, Heparin, Kalium, H2-Blocker, Bicarbonat } \\
\text { - nicht vasoaktive Medikamente, Diuretika etc. (siehe andere TISS-Items) }\end{array}$ & $\begin{array}{l}\text { Ein Med. =2 } \\
\text { zwei od. } \\
\text { mehr Med. = } 3\end{array}$ \\
\hline $\begin{array}{l}\text { Verbands- } \\
\text { Wechsel }\end{array}$ & $\begin{array}{l}\text { - Verbandswechsel (auch Dekubituspflege) } \\
\text { - Routine heißt } 1 \text { - } 2 \text { Mal täglich } \\
\text { - häufig heißt mind. } 3 \text { Mal täglich und/oder ausgedehnte Wundpflege }\end{array}$ & $\begin{array}{c}\text { Routine }=1 \\
\text { häufig }=2\end{array}$ \\
\hline Drainagen & - Pflege aller Drainagen, auch Spül-Saug-Drainage (nicht Magensonde) & $\mathbf{3}$ \\
\hline \multicolumn{3}{|l|}{ Lunge } \\
\hline \multirow[t]{2}{*}{ Beatmung } & $\begin{array}{l}\text { - jegliche Beatmungsform mit maschineller Unterstützung, mechanisch/ } \\
\text { assistiert, auch Spontanatmung mit PEEP }\end{array}$ & 5 \\
\hline & $\begin{array}{l}\text { - Atmungsunterstützung: Spantanatmung über Tubus ohne PEEP, 02- } \\
\text { Maske oder -Nasenschlauch }\end{array}$ & 2 \\
\hline $\begin{array}{l}\text { Künstliche } \\
\text { Luftwege }\end{array}$ & $\begin{array}{l}\text { - Pflege der künstlichen Luftwege } \\
\text { - Endotracheal-Tubus, Tracheostoma }\end{array}$ & 1 \\
\hline Atemtherapie & $\begin{array}{l}\text { - Behandlung zur Verbesserung der Lungenfunktion: Physiotherapie } \\
\text { (Atemgymnastik), endotracheales Absaugen, aktive Vernebelung über } \\
\text { Tubus oder Maske }\end{array}$ & 1 \\
\hline \multicolumn{3}{|l|}{ Herz-Kreislauf } \\
\hline $\begin{array}{l}\text { Vasoaktive } \\
\text { Medikamente }\end{array}$ & $\begin{array}{l}\text { - Katecholamine: jedes Medikament, jede Dosis } \\
\text { - z. B. Adrenalin, Noradrenalin, Dobutamin, Dopamin, Dopexamin, } \\
\text { Neosynephrin und Analoga, nicht Nitrospray, ACE-Hemmer }\end{array}$ & $\begin{array}{l}\text { ein Med. }=3 \\
\text { mind. zwei Med. } \\
\quad=5\end{array}$ \\
\hline $\begin{array}{l}\text { Flüssigkeits- } \\
\text { Therapie }\end{array}$ & $\begin{array}{l}\text { - großer Volumenersatz i.v., mind. } 6 \text { Liter pro } 24 \mathrm{H}\left(>3 \mathrm{l} / \mathrm{m}^{2} \text { pro } 24 \mathrm{~h} \text { ) }\right. \\
\text { (Gesamtmenge incl. Blutprodukten, aber nicht bei Hämodialyse) }\end{array}$ & 4 \\
\hline Arterie & - peripherer arterieller Katheter & 5 \\
\hline Pulmonaliskath. & - Pulmonaliskatheter mit oder ohne Cardiac Output Messung & 8 \\
\hline ZVK & - zentral-venöser Katheter & 2 \\
\hline Reanimation & $\begin{array}{l}\text { - kardiopulmonale Reanimation nach Herzstillstand } \\
\text { (nicht einzelner präkordialer Faustschlag) }\end{array}$ & 3 \\
\hline \multicolumn{3}{|c|}{ 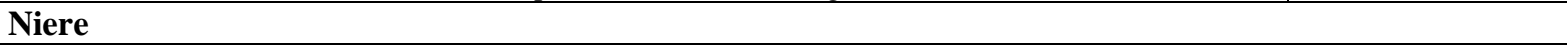 } \\
\hline Dialyse & - Hämofiltration, Dialyse (diverse Techniken) & 3 \\
\hline Ausfuhr & - quantitative Bestimmung der Urinmenge (mit oder ohne Urinkatheter) & 2 \\
\hline Diurese & $\begin{array}{l}\text { - aktive medikamentös unterstütze Diurese } \\
\text { (z. B. Furosemid > } 1 \mathrm{mg} / \mathrm{kg} \mathrm{KG} \text { oder analoge Medikation) }\end{array}$ & 3 \\
\hline \multicolumn{3}{|c|}{ (t) } \\
\hline ICP & - Messung des intrakraniellen Druckes & 4 \\
\hline \multicolumn{3}{|l|}{ Metabolismus } \\
\hline $\begin{array}{l}\text { Azidose/ } \\
\text { Alkalose }\end{array}$ & $\begin{array}{l}\text { - Behandlung einer entgleisten metabolischen Azidose/Alkalose oder } \\
\text { - mindestens } 2 \mathrm{mval} / \mathrm{kg} \mathrm{KG} \text { Bikarbonat }\end{array}$ & 4 \\
\hline Ernährung & - i.v. Alimentation mit Glukose UND Aminosäuren, ggf. auch Lipide & 3 \\
\hline $\begin{array}{l}\text { Enterale } \\
\text { Ernährung }\end{array}$ & - mind. 500 ml/Tag Sondenkost über Magensonde oder PEG (et sim.) & 2 \\
\hline \multicolumn{3}{|l|}{ Interventionen } \\
\hline $\begin{array}{l}\text { Interventionen } \\
\text { Auf der ICU }\end{array}$ & $\begin{array}{l}\text { - z. B. endotracheale Intubation, Einsetzen eines Schrittmachers, } \\
\text { Kardioversion, Endoskopie, Notfall-OP auf ICU, Magenspülung, TEE, } \\
\text { Bronchoskopie } \\
\text { - keine Routineinterventionen wie etwa Röntgen-Thorax }\end{array}$ & $\begin{array}{l}\text { eine Interv. =3 } \\
\text { mind. } \text { zwei }=5\end{array}$ \\
\hline $\begin{array}{l}\text { Interventionen } \\
\text { außerhalb der ICU }\end{array}$ & $\begin{array}{l}\text { - besondere Interventionen außerhalb der Intensivstation } \\
\text { - Operationen oder Diagnostik (z. B. CT) }\end{array}$ & 5 \\
\hline
\end{tabular}

Tabelle 2: Die Zusammensetzung des TISS - 28 - Scores aus den einzelnen Parametern (nach Miranda et al. 1996, S. 65). 
Zunächst werden in dem Score die Basistätigkeiten bestimmt, wie Standardmonitoring mit stündlichen Vitalzeichenkontrollen und regelmäßigen Berechnungen der Flüssigkeitsbilanz, die Bestimmung laborchemischer und mikrobiologischer Parameter, die Applikationsform und Dosierung eines oder mehrerer Medikamente, routinemäßige oder häufige Verbandswechsel und die Pflege von Drainagen. Des Weiteren werden die verschiedenen Formen der Beatmung, die Anlage und die Pflege der künstlichen Luftwege sowie die Art der erfolgten Atemtherapie punktemäßig erfasst. In die Erfassung der Herz - Kreislauf - Maßnahmen fließen die Applikation vasoaktiver Medikamente, die Flüssigkeitstherapie, das Vorhandensein eines zentralvenösen oder arteriellen Zugangs bzw. eines Pulmonaliskatheters sowie eine stattgefundene Reanimation ein. Weitere Punkte werden bei der Bewertung des renalbedingten Aufwandes vergeben, wobei die Dialyse, die quantitative Bestimmung der Urinmenge und die medikamentös unterstützte Diurese für den Score von Relevanz sind. Ferner gehen die Anlage zur Messung des intrakraniellen Drucks, die Behandlung einer Azidose oder Alkalose, die intravenöse Ernährung und die enterale Ernährung mittels Magensonde oder PEG - Sonde in die Punktevergabe des TISS - 28 - Scores ein. Schließlich werden weitere Interventionen, welche innerhalb oder auch außerhalb der Intensivstation erfolgen können, erfasst. Dies betrifft $u$. a. die endotracheale Intubation, die Schrittmacherimplantation, die Kardioversion, Endoskopien, Bronchoskopien, spezielle Ultraschalluntersuchungen (z. B. TEE), spezielle Röntgenuntersuchungen (z. B. CT) oder Notfalloperationen. Routineinterventionen, wie beispielsweise das Röntgen der Lunge, Ultraschalluntersuchungen oder EKG, werden unter dem Punkt weitere Interventionen im TISS - 28 nicht berücksichtigt.

Die sich aus den obigen Parametern ergebende Gesamtpunktzahl des TISS - 28 - Scores ist auch ein wichtiges Instrument zur Personalberechnung im Pflegedienstmanagement (Miranda et al. 1996). Auch in der vorliegenden Studie kommt der TISS - 28 - Score zur Anwendung. 


\subsection{Das Patientenkollektiv und die Datenerfassung aus der Patientenakte in dieser Studie}

Nach Prüfung und Genehmigung durch die Ethikkommission der Medizinischen Fakultät (Antragsnummer 6/2/04) und dem Einverständnis der Leitung der Abteilung für Kardiologie und Pneumologie im Zentrum Innere Medizin wurden in unsere Studie Patienten einbezogen, die sich im Zeitraum von Juni bis Oktober 2004 in stationärer Behandlung auf der Intensivstation 1026 des Universitätsklinikums Göttingen befanden. Bei dieser Station handelt es sich um eine schwerpunktmäßig kardiologische Intensivstation, welche vom Zentrum Innere Medizin geführt wird. Dieses Zentrum, u.a. bestehend aus den Disziplinen Kardio - und Pneumologie, Nephro - und Rheumatologie, Hämato - und Onkologie und Gastroentero - und Endokrinologie, stellt auch das ärztliche Personal zur Behandlung der Patienten. Nach dem Aufenthalt auf der Intensivstation werden die Patienten in der Regel auf die internistischen Normalstationen verlegt.

Einschlusskriterien für diese Studie waren das vollendete 18. Lebensjahr und eine intensivstationäre Mindestaufenthaltsdauer von 24 Stunden. Primär wurden keine Patienten, welche die Einschlusskriterien erfültten, aus der Studie ausgeschlossen. Demnach waren die Ausschlusskriterien das nicht vollendete 18. Lebensjahr und eine Aufenthaltsdauer von weniger als 24 Sunden auf der Intensivstation.

Unter Berücksichtigung dieser Kriterien wurden in das Kollektiv täglich alle neu aufgenommenen Patienten des Vortages eingeschlossen. War dieses aus Gründen mangelnder Erfassungskapazitäten einmal nicht möglich, wurde randomisiert und nach dem Zufallsprinzip ausgewählt, welche der Patienten in die Studie aufgenommen werden. Insgesamt wurden in dieser Studie die Daten von 100 internistischen Intensivpatienten analysiert.

Die umfangreiche Erfassung aller für diese Studie notwendigen Daten erfolgte täglich aus der Patientenakte. Patientendaten, ärztliche Anordnungen, durchgeführte Prozeduren sowie pflegerische und physiotherapeutische Maßnahmen werden hier dokumentiert. Die an den 
Intensivbetten befindlichen Patientenmonitore und Geräte zur Überwachung der Vitalparameter, wie z. B. Blutdruckmessgeräte oder Pulsoximeter, erheben in regelmäßigen Abständen Werte für Herzfrequenz, Blutdruck und Sauerstoffsättigung, welche graphisch in die Patientenakte übertragen werden. Es besteht somit jederzeit die Möglichkeit, verschiedene Parameter aus der Akte zu ersehen.

Ferner werden in der Patientenakte Befunde durchgeführter Interventionen, die täglichen Laborwerte und mikrobiologische Befunde abgeheftet.

\subsection{Die Software zur Datenerfassung in dieser Studie}

Für die standardisierte Erfassung der Patientendaten in der bereits in der Einleitung erwähnten multizentrischen Studie, deren Bestandteil die vorliegende Untersuchung ist, entwickelte die Fa. HealthEcon AG Basel, das DIVI - COSt - Computerprogramm. Hierbei handelt es sich um eine Datenbank auf der Grundlage des Statistikprogramms Microsoft Access. Mittels dieser Software wurden die Daten der vorliegenden Studie unter den Aspekten Ressourcenverbrauch und Scores erhoben und ausgewertet.

Das DIVI - COSt - Computerprogramm besteht aus einer katalogähnlichen Auflistung sämtlicher Materialien und Leistungen, welche mit Kosten für das Krankenhaus verbunden sind. Dieser Teil der Software stand unter dem Oberbegriff „Ressourcenverbrauch/ Leistungserhebung" und beinhaltete die folgenden Unterpunkte in vorgegebenen Masken: „Medikamente“, „Perfusortherapie“, „Blutprodukte und Gerinnungsfaktoren“, „Volumenersatz und Basisinfusionen“, „Ernährung und Supplemente“, "Beatmung“, "Invasive Maßnahmen / Monitoring“, "Sonstige Maßnahmen / Diagnostik", "Laboranalysen und Mikrobiologie“. Die einzelnen Masken beinhalten freie Felder, in welche Begriffe und numerische Werte eingetragen werden können. Diese wurden mit patientenspezifischen Daten ausgefüllt und dokumentieren somit die täglich verbrauchten Ressourcen und erbrachten Leistungen. War eine 
erbrachte und somit zu erfassende Leistung bisher nicht im vorgegebenen Katalog enthalten, konnte das Programm jederzeit um diesen Punkt erweitert und die Leistung fortan dokumentiert werden.

Aus den folgenden Abbildungen 1-10 wird deutlich, wie die Patientendaten bezüglich der täglich verbrauchten Ressourcen und erbrachten Leistungen aus der aktuellen stationären Patientenakte in die zugehörigen Abschnitte des DIVI - COSt - Computerprogramms übernommen wurden.

\subsubsection{Die Datenerfassung der verbrauchten Ressourcen und erbrachten Leistungen in den einzelnen Masken des DIVI - COSt - Computerprogramms}

\subsubsection{Medikamente}

Bei der Dokumentation der eingesetzten Medikamente wurden zunächst die Handelsnamen mit den Dosierungen der Präparate angegeben. Es folgten Informationen zur jeweils verabreichten Einzeldosis des Medikaments samt der Einheit in [mg], [ml], [mval], [IE] oder Tropfen.

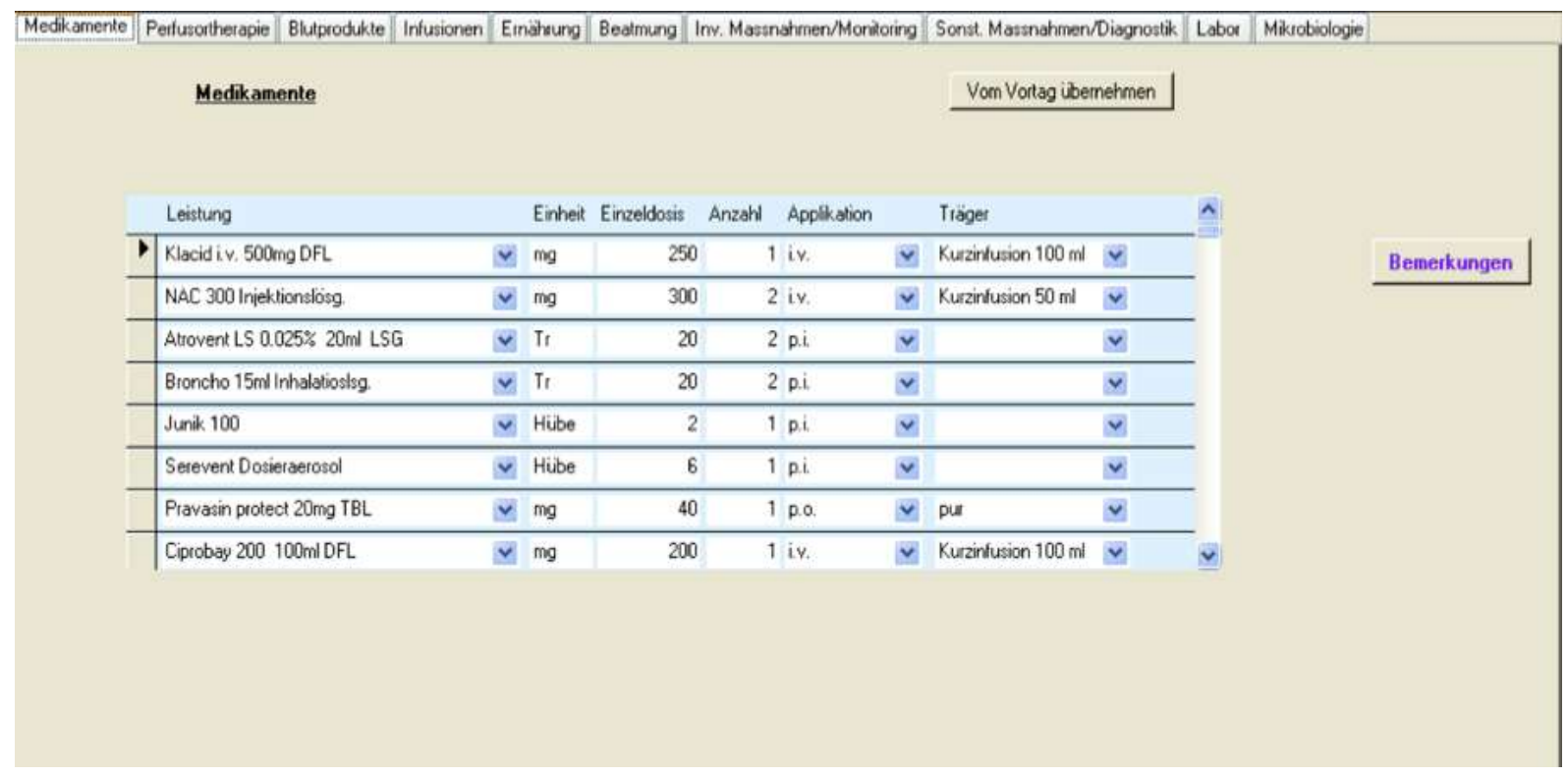

Abbildung 1: Die ausgefüllte Bildschirmmaske des Abschnitts „Medikamente“ im DIVI - COSt - Computerprogramm. 
Weiterhin wurde die Art der Applikation, welche i. v., s. c., p. o. oder rect. erfolgen kann, angegeben. Unter dem Begriff „Träger“ wurde in der letzten Spalte aufgeführt, ob ein Medikament pur oder in einer Trägersubstanz, wie z. B. Kochsalzlösung, verabreicht wurde.

\subsubsection{Perfusortherapie}

Zur Erfassung der mittels Perfusor applizierten Medikamente wurde zunächst wieder der Handelsname des Präparates mit seiner Dosierung angegeben. Es folgte die Angabe der Einheit des Stoffes in [mg], [ml], [mval] oder [IE]. In einer weiteren Spalte wurde die Anzahl der durchgelaufenen Perfusorspritzen (auf die erste Nachkommastelle gerundet) dokumentiert.

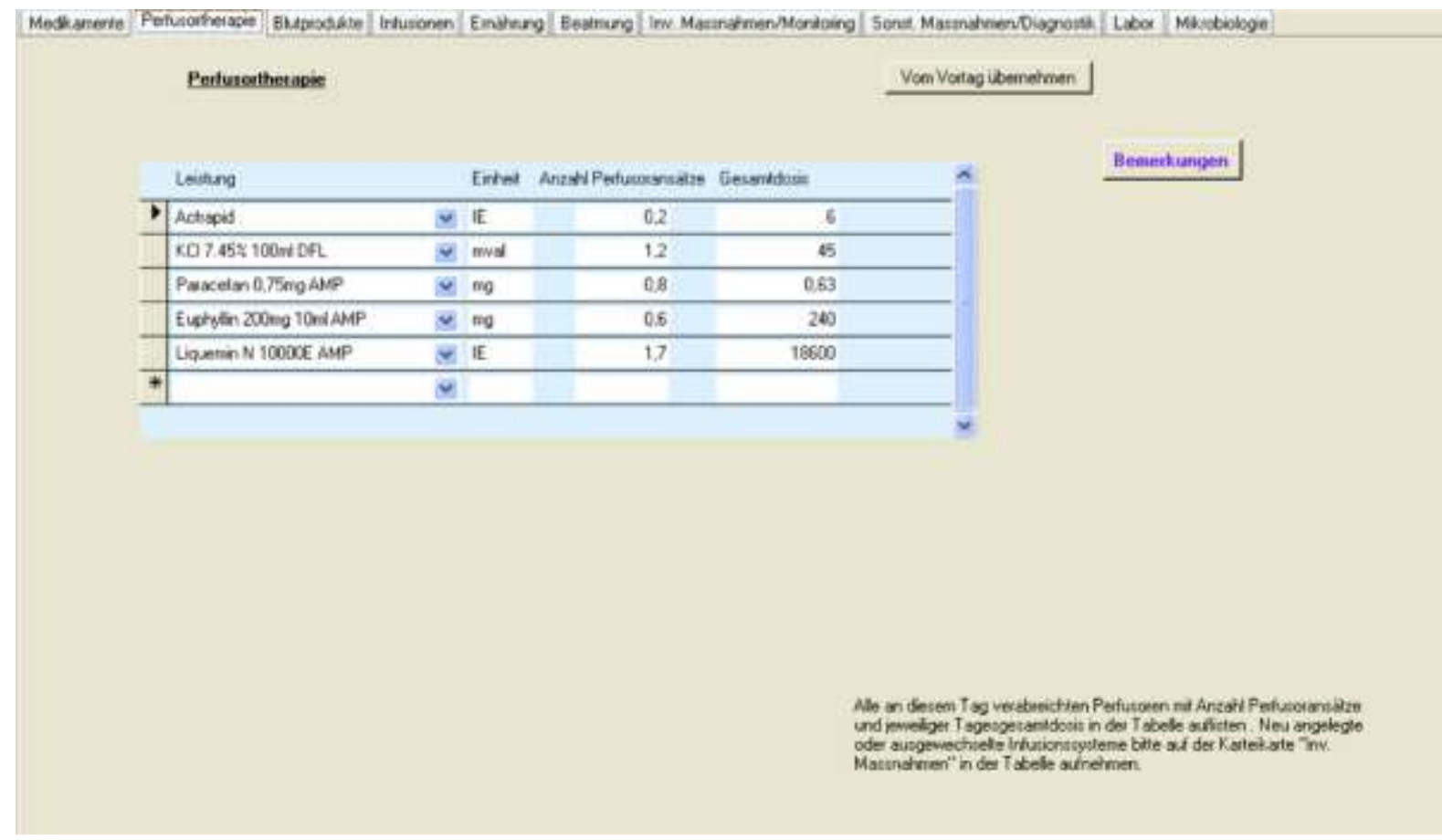

Abbildung 2: Die ausgefüllte Bildschirmmaske des Abschnitts „Perfusortherapie“ im DIVI - COSt - Computerprogramm. 


\subsubsection{Blutprodukte und Gerinnungsfaktoren}

Die Erfassung der verabreichten Blutprodukte wurde durch die Angabe der Art des Produktes und der Volumenmenge in [ml], in der es pro Stück abgepackt zur Verfügung stand, dokumentiert. Des Weiteren wurde in einer Spalte die Stückzahl einer Applikation und in einer weiteren Spalte die Gesamtzahl der Applikationen erfasst.

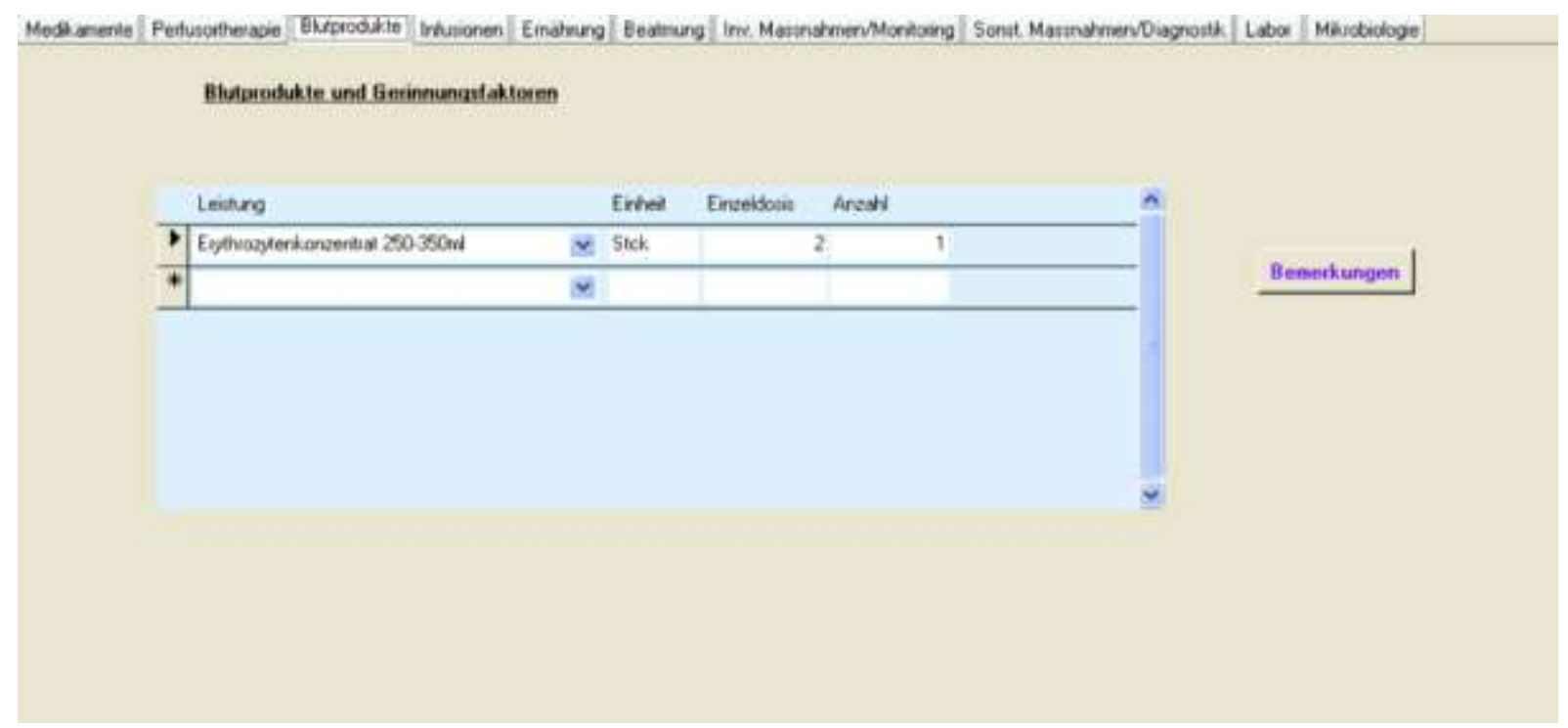

Abbildung 3: Die ausgefüllte Bildschirmmaske des Abschnitts „Blutprodukte und Gerinnungsfaktoren“ im DIVI - COSt - Computerprogramm. 


\subsubsection{Volumenersatz und Basisinfusionen}

In diesem Abschnitt erfolgte die Erfassung der verbrauchten Infusionen, indem in der ersten Spalte der Handelsname mit der Dosierung des infundierten Präparates aufgeführt wurde. In der nächsten Spalte wurde die Anzahl der verabreichten Infusionen bis auf eine Nachkommastelle gerundet und in der letzten Spalte das Tagesvolumen der Infusion in [ml] angegeben.

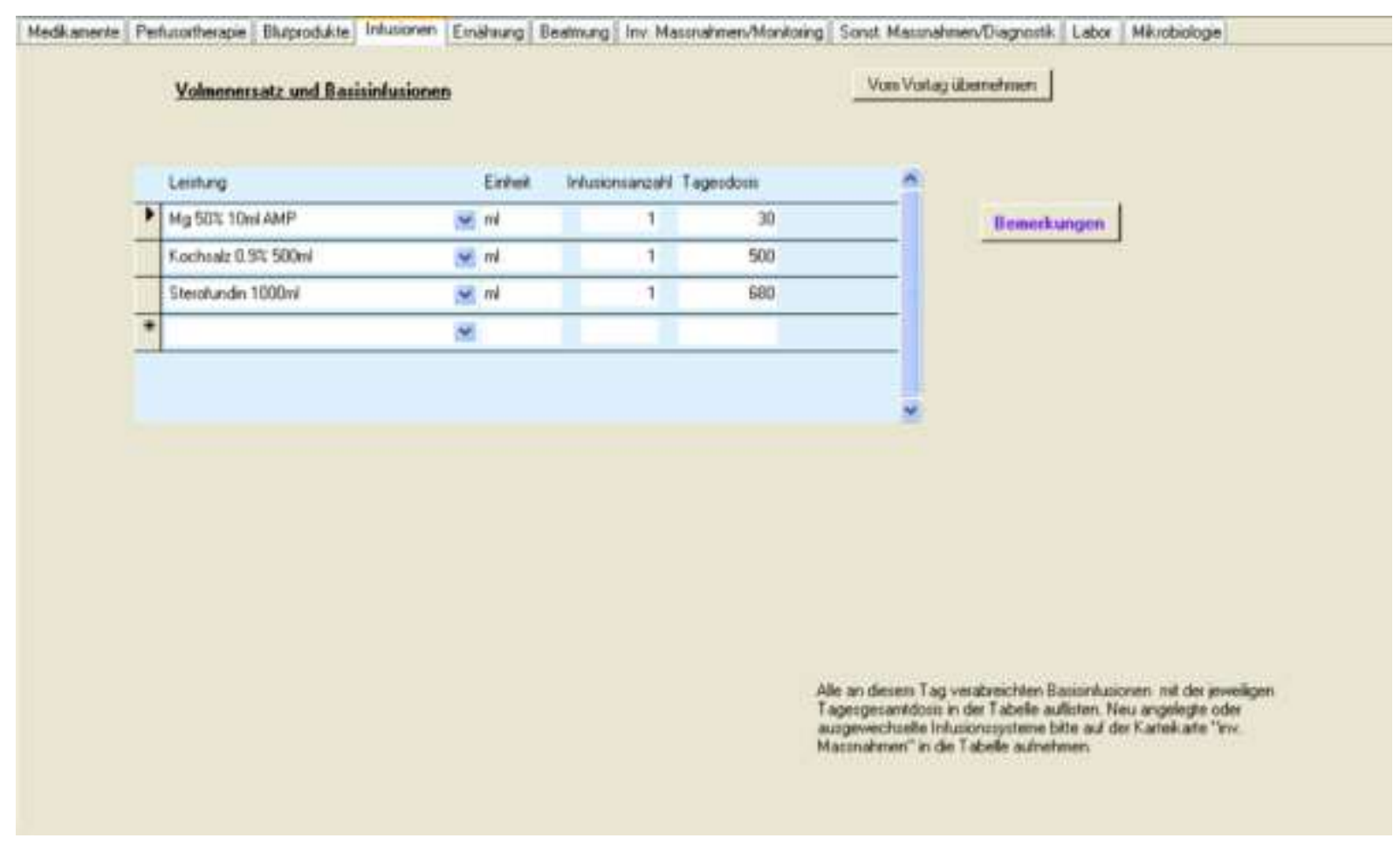

Abbildung 4: Die ausgefüllte Bildschirmmaske des Abschnitts „Volumenersatz und Basisinfusionen" im DIVI - COSt - Computerprogramm. 


\subsubsection{Ernährung und Supplemente}

Die Ernährung wurde entweder durch ein Häkchen in den Feldern "Normalkost“ oder "Nahrungskarenz" oder durch das Auflisten der verbrauchten Einzelressourcen bei parenteraler oder enteraler Ernährung in der dafür angelegten Tabelle dokumentiert. Ein Häkchen bei „Nahrungskarenz" bedeutete für diesen Tag keinerlei Ressourcenverbrauch und somit auch keinerlei Kosten. Wurde die Ernährung per Magensonde oder parenteral verabreicht, mussten die Präparate mit Handelsnamen, Einzeldosen in [ml] und der Gesamtzahl der Applikationen angegeben werden.

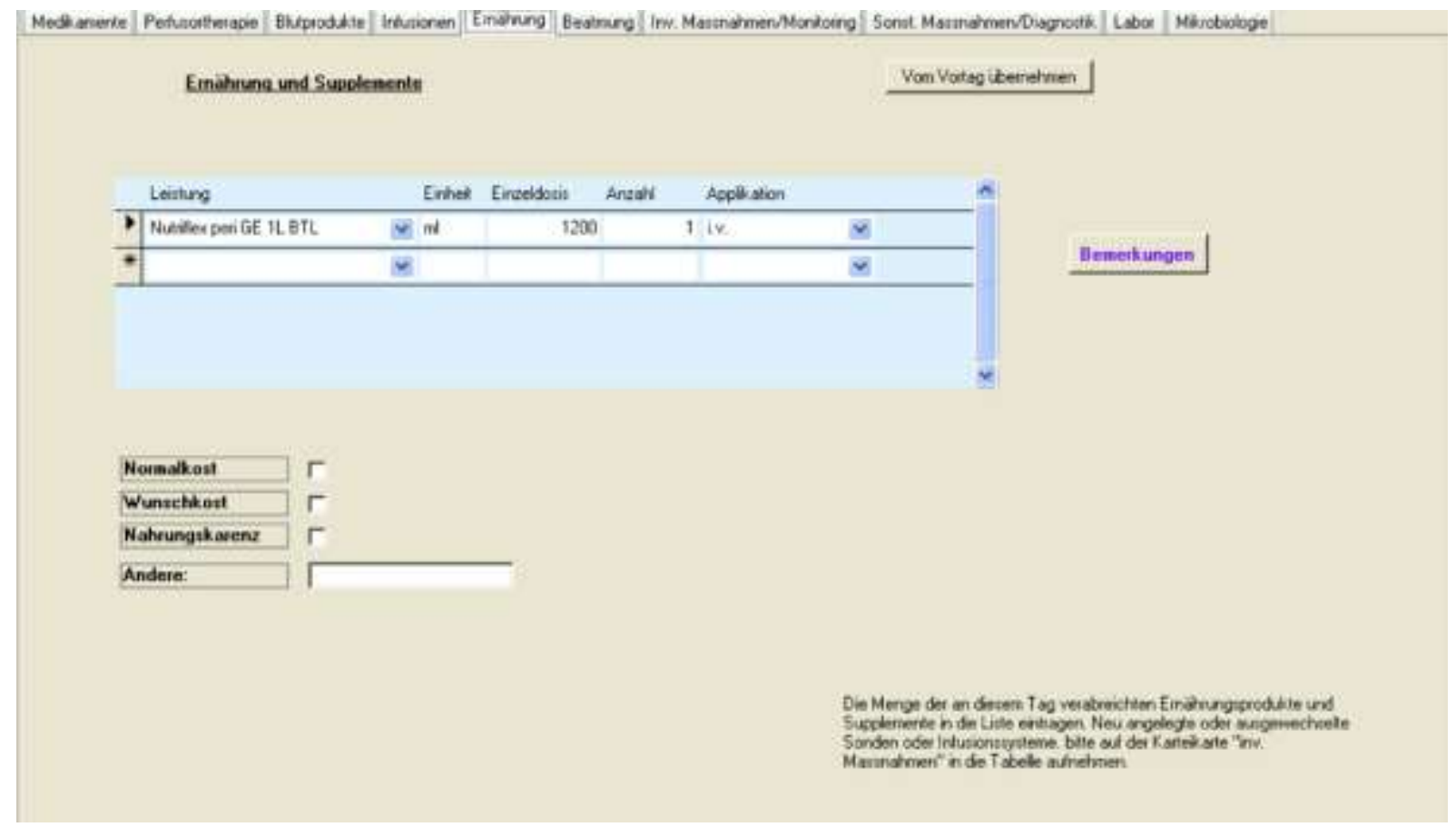

Abbildung 5: Die ausgefüllte Bildschirmmaske des Abschnitts „Ernährung und Supplemente" im DIVI - COSt - Computerprogramm. 


\subsubsection{Beatmung}

In diesem Abschnitt wurde zunächst bei der Dokumentation beatmeter Patienten zwischen invasiver Beatmung mit Tubus oder Tracheostoma und nicht invasiver Beatmung mit Maske oder Helm unterschieden. Bei Patienten, die Sauerstoff erhielten, wurde zwischen der Zufuhr mittels Maske oder Brille differenziert. Ferner wurde bei beatmeten Patienten der Beatmungsmodus festgehalten, wobei als Modi CPAP-ASB, BiPAP und SIMV zur Verfügung standen. Weiterhin wurde erfasst, ob die Beatmung oder Sauerstoffgabe permanent oder intermittierend erfolgte und die Zeitdauer in Stunden angegeben. Schließlich wurden die neu hinzugekommenen oder ausgetauschten Anlagen, die für die Beatmung notwendig waren, wie Beatmungsfilter oder ein neuer Tubus, dokumentiert. Auch die Häufigkeit des endotrachealen Absaugens wurde erfasst.

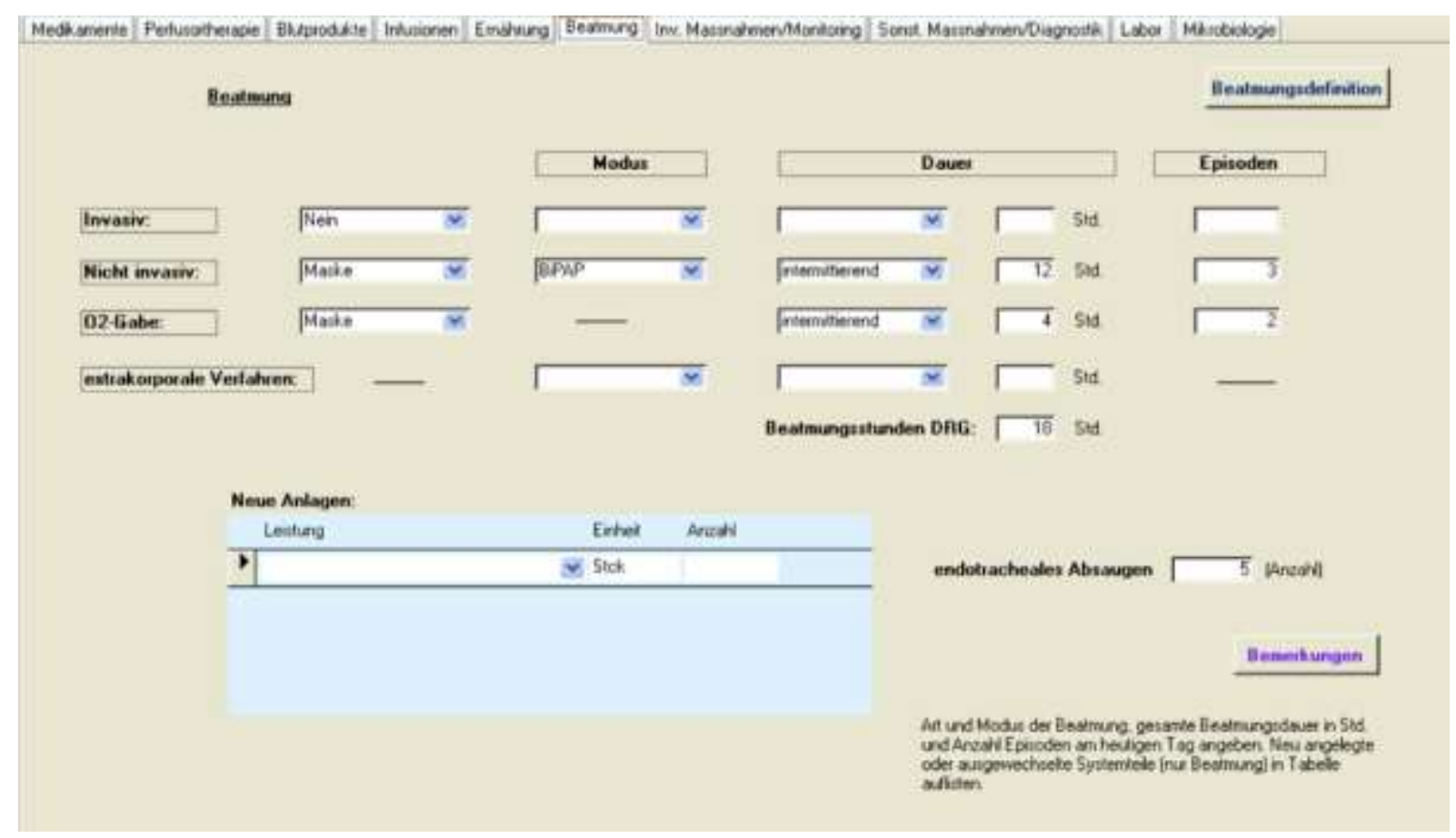

Abbildung 6: Die ausgefüllte Bildschirmmaske des Abschnitts „Beatmung“ im DIVI - COSt - Computerprogramm. 


\subsubsection{Invasive Maßnahmen / Monitoring}

In einer katalogartig vorgegebenen Liste der invasiven Maßnahmen wurde zunächst durch Einsetzen eines Häkchens in der Spalte „"äuft" dokumentiert, ob ein Patient über ein System zur Messung des zentralvenösen Druckes, des intrakraniellen Druckes, einen Dauerkatheter, einen arteriellen Zugang oder einen Pulmonaliskatheter verfügte und ob ein Nierenersatzverfahren angewendet wurde. Der Wechsel einer der obigen Anlagen wurde mit einem Häkchen in der nächsten Spalte dokumentiert und in der darunter stehenden Tabelle mit Angabe der gewechselten Stückzahl aufgeführt. In der Folge wurde der genaue Typ der mechanischen Kreislaufunterstützung und die genaue Verfahrensweise samt Filtermenge des Nierenersatzes eingetragen.

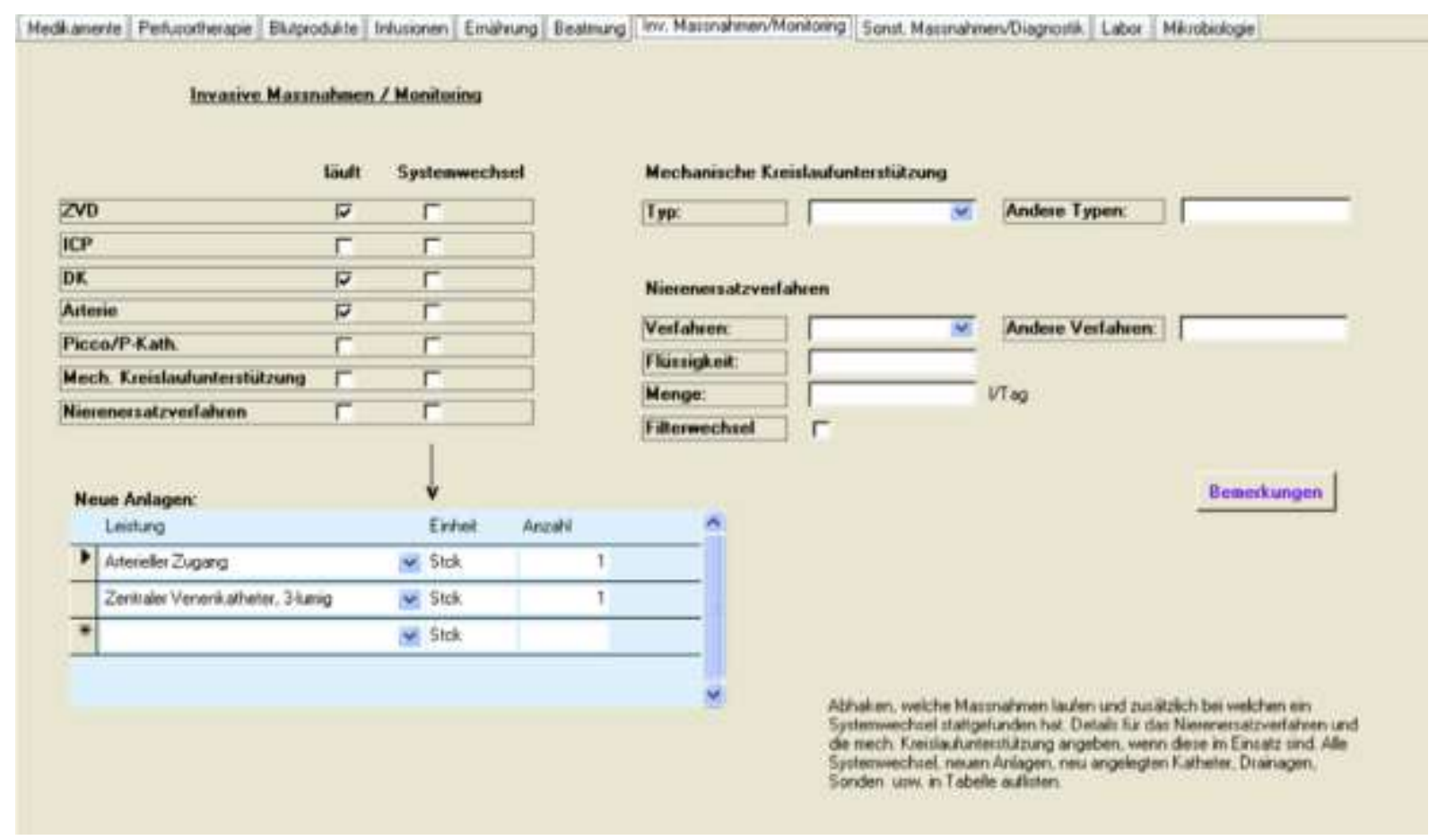

Abbildung 7: Die ausgefüllte Bildschirmmaske der Abschnitts „Invasive Maßnahmen / Monitoring“ im DIVI - COSt - Computerprogramm. 


\subsubsection{Sonstige Maßnahmen / Diagnostik}

In der Tabelle „Pflegerische Maßnahmen“ wurden die bei der Pflege der Patienten verbrauchten Materialien aufgeführt. Die zur Mobilisation der Patienten angewandten Maßnahmen wurden unter dem Punkt "Physiotherapie" erfasst. Auch eine Isolationspflege, welche beispielsweise bei einer MRSA - Infektion eines Patienten notwendig ist, wurde hier vermerkt. Zur Erfassung diagnostischer Untersuchungen, wie EKG, röntgenologische- und computertomographische Aufnahmen, Bronchoskopien oder andere endoskopische Untersuchungen und ihrer Anzahl, diente eine darunter stehende Tabelle. Des Weiteren konnten in der untersten Tabelle auch zusätzlich vorgenommene Operationen und Prozeduren, wie beispielsweise Untersuchungen des Liquorsystems, dokumentiert werden.

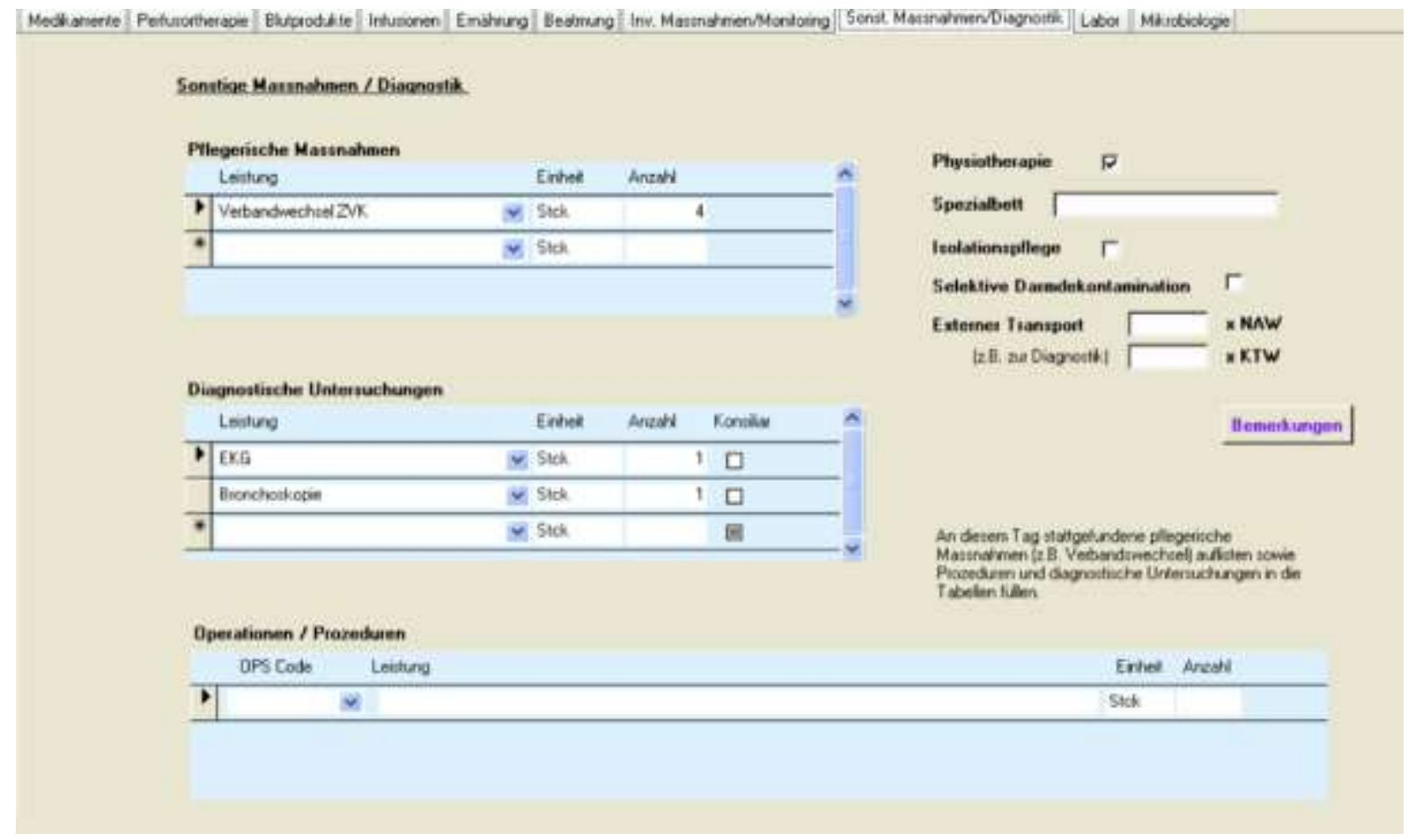

Abbildung 8: Die ausgefülte Bildschirmmaske des Abschnitts „Sonstige Maßnahmen / Diagnostik“ im DIVI - COSt - Computerprogramm. 


\subsubsection{Laboranalysen}

Hinter jedem der aufgelisteten laborchemischen Parameter befand sich ein freies Feld, in welches die Gesamtzahl der Bestimmungen dieser Werte am betreffenden Tag eingetragen wurde. Wurden Laborwerte bestimmt, welche nicht in der vorgegebenen Auflistung der Software vorhanden waren, konnten diese in einer Spalte für sog. weitere Laboranalysen manuell eingefügt werden.

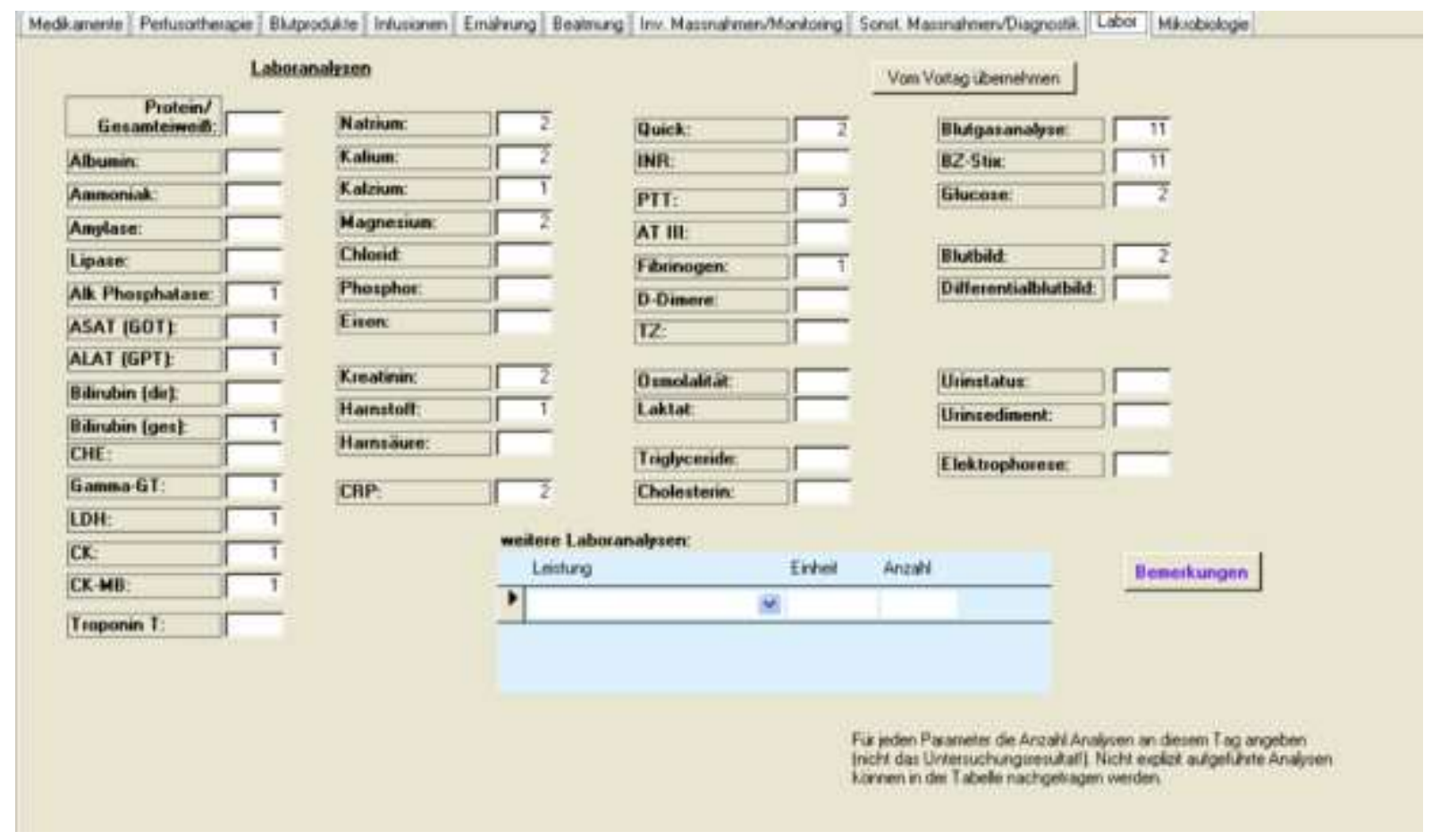

Abbildung 9: Die ausgefüllte Bildschirmmaske des Abschnitts „Laboranalysen“ im DIVI - COSt - Computerprogramm. 


\subsubsection{Mikrobiologie}

Im letzten Abschnitt wurden alle mikrobiologisch untersuchten Materialien erfasst. Zunächst wurde das zu untersuchende Material aufgeführt. Falls sich bei einem positiven mikrobiologischen Befund weitere Untersuchungen zur Differenzierung der Erreger sowie der Erstellung eines Antibiogramms und somit weitere Kosten anschlossen, wurde dies durch Setzen eines Häkchens dokumentiert.

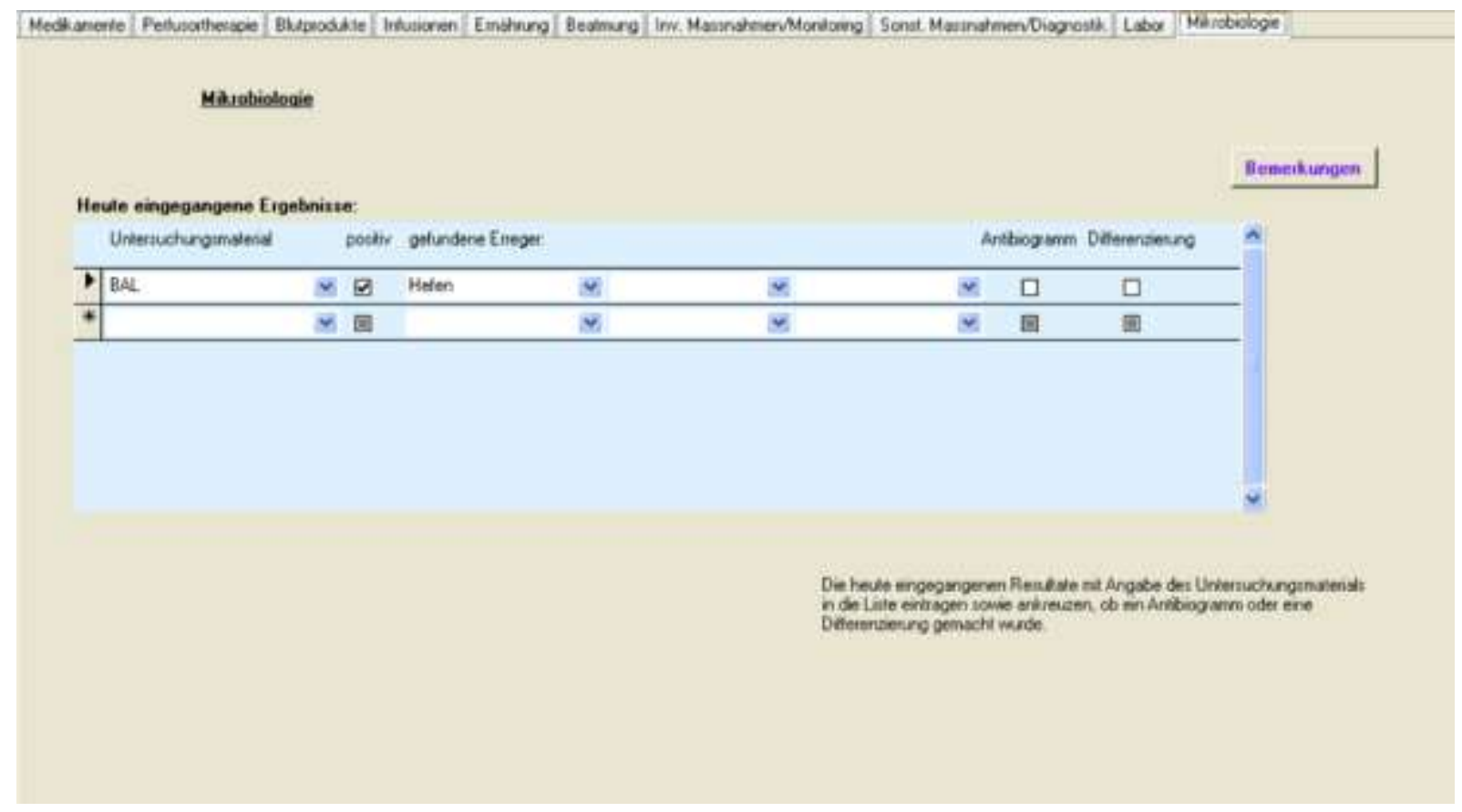

Abbildung 10: Die ausgefüllte Bildschirmmaske des Abschnitts "Mikrobiologie“ im DIVI - COSt - Computerprogramm. 


\subsubsection{Die Dokumentation der Scores SAPS II und TISS - 28}

Um den SAPS II - Score zu erheben, wurden für dessen Parameter (siehe Kapitel 2.1.), welche im DIVI - COSt - Computerprogramm aufgelistet waren, die jeweils pathologischsten Werte der letzten 24 Stunden manuell in die freien Felder eingetragen. Die Software verfügte über eine Funktion der automatischen Berechnung der Scorewerte für die einzelnen Parameter sowie der Berechnung des Gesamtscores.

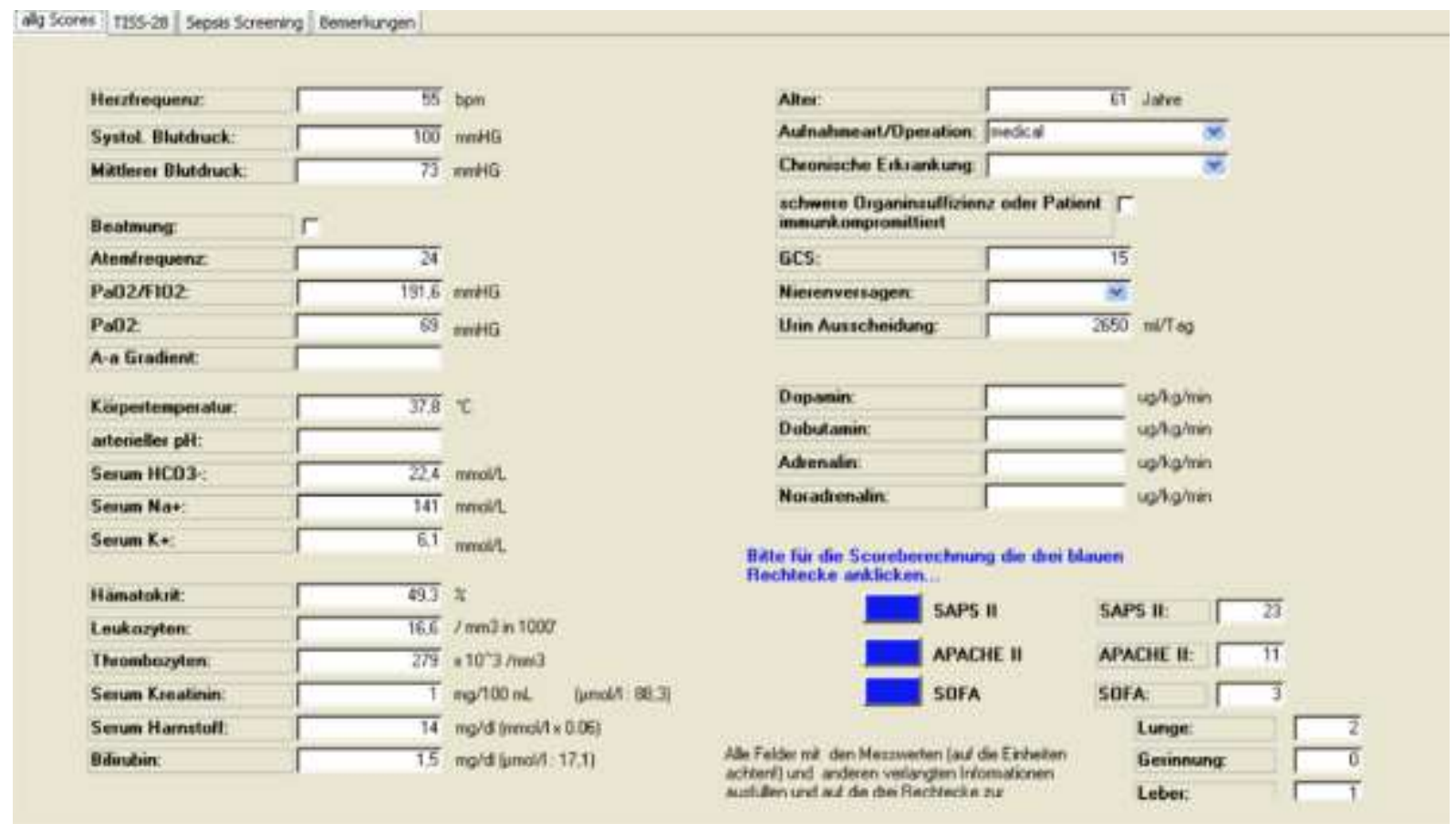

Abbildung 11: Die ausgefüllte Bildschirmmaske des Abschnitts „Erhebung und Berechnung des SAPS II“ im DIVI - COSt - Computerprogramm. 
Für die Dokumentation des TISS - 28 (siehe Kapitel 2.1.) wurde der pflegerische Aufwand durch Setzen von Häkchen in die vorgegebene Liste der katalogartig aufgeführten Tätigkeiten erfasst. Ähnlich wie für den oben genannten Score funktionierte die automatische Berechnung des TISS - 28 - Score durch das DIVI - COSt - Computerprogramm, wobei jede pflegerische Tätigkeit einem Zahlenwert entsprach und die Software schließlich die Einzelwerte zum Gesamtscore addierte.

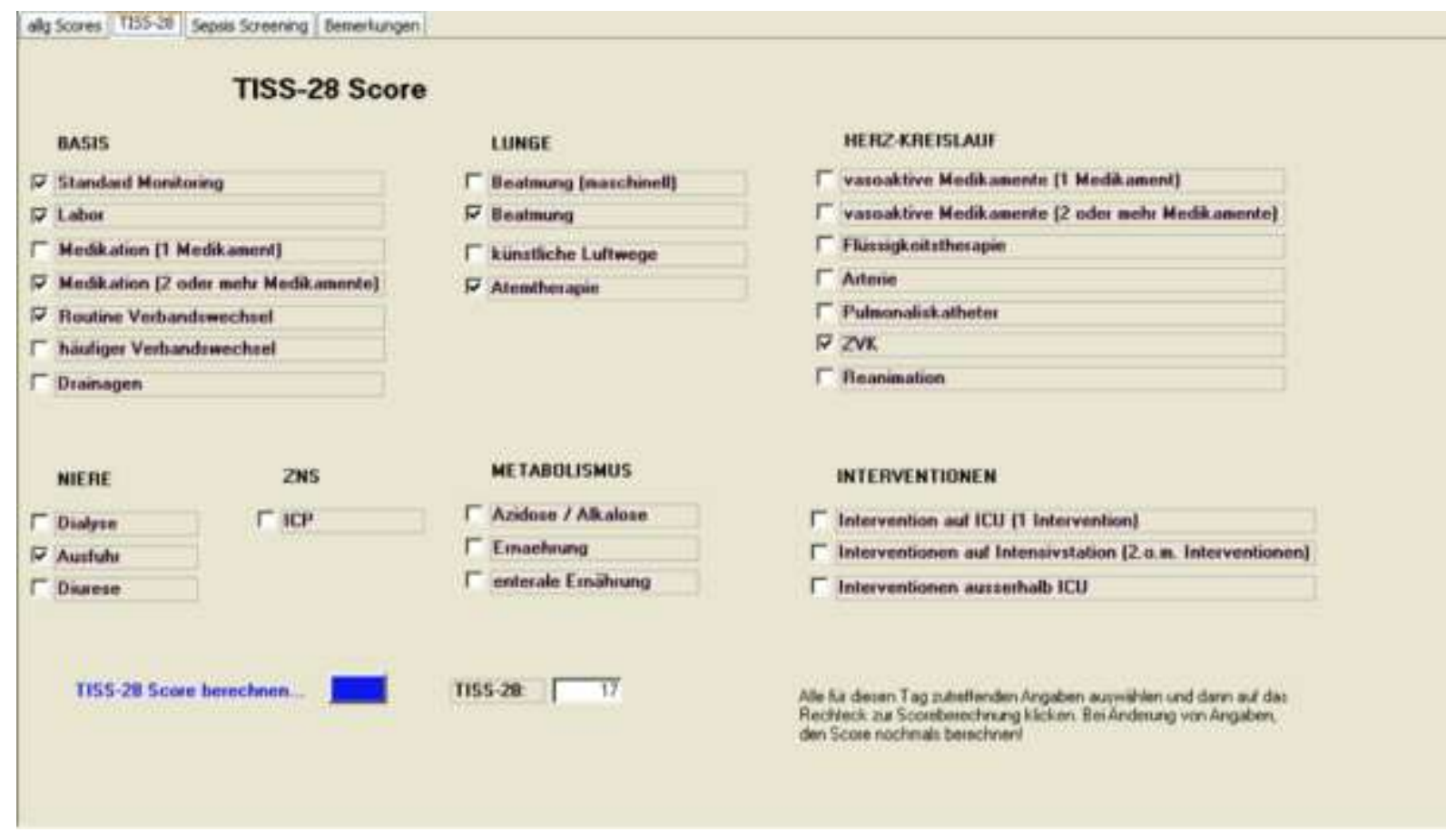

Abbildung 12: Die ausgefüllte Bildschirmmaske des Abschnitts „Erhebung und Berechnung des TISS - 28“ im DIVI - COSt - Computerprogramm. 


\subsubsection{Personal - und Overheadkosten}

In diesem Kapitel geht es darum, die allgemeinen, patientenunspezifischen Kosten zu erheben. Dabei wurden in Zusammenarbeit mit der Leitung der Abteilung Anaesthesiologie II und der Verwaltung des Universitätsklinikums Göttingen zunächst die ausgeübte Funktion und Anzahl sämtlicher Mitarbeiter sowie deren Gehaltsgruppenzugehörigkeit erfasst, um die Personalkosten zu ermitteln. Hierfür wurde dokumentiert, wie viele Chef -, Ober -, und Assistenzärzte, Personen des Pflegepersonals, Physiotherapeuten, Sekretärinnen und weitere Personen im Bereich der Intensivstation direkt beschäftigt sind.

Auf das Jahr 2003 bezogen entstanden demnach Personalkosten in Höhe von 1.857.800 Euro insgesamt. Diese beinhalten jedoch nur die Personalkosten der Personen, welche eindeutig der Intensivstation 1026 zuzuordnen sind. In deren Personalausstattung sind 8 Assistenzärzte (vollzeitlich auf der Station 1026 beschäftigt) und 28 Pflegekräfte (ganze Stellen) verzeichnet.

Bei einer Bettenzahl von 9 Betten ergeben sich 3.285 Pflegetage im Jahr. Werden nun die gesamten Personalkosten durch die in dem Jahr geleisteten 3.285 Pflegetage dividiert, ergeben sich somit Personalkosten von 565,54 Euro pro Patient und Tag.

Zusätzlich müssen noch die Kosten eines Oberarztes (33\% der Arbeitszeit in der Frühschicht auf der Station 1026 beschäftigt) und anderer diensthabender Oberärzte in der Spät - und Nachtschicht mit jeweils 33\% berücksichtigt werden. Insgesamt ist also ein Oberarzt mit 100\% hinzu zu rechnen. Die Kosten dieses Oberarztes werden mit 71.100 Euro pro Jahr angesetzt. Dividiert man diese wiederum durch die 3.285 Pflegetage, so ergeben sich zusätzliche Tageskosten von 21,64 Euro pro Tag.

Des Weitern sind 2 Physiotherapeuten insgesamt 4 Stunden pro Tag auf der Station tätig. Dies entspricht $50 \%$ der Personalkosten einer Physioherapeutenstelle. Bei Gehaltskosten von 43.700 Euro für einen Physiotherapeuten sind somit 21.850 Euro wieder durch die 3.285 Pflegetage im Jahr zu teilen. Somit ergeben sich weitere zusätzliche Tageskosten von 6,65 Euro pro Tag. 
Für die sog. Overhead - Kosten, welche unter anderem Ausgaben wie Energie - und Reinigungskosten umfassen, wurde der tagesbezogene Basispflegesatz, den die Verwaltung des Universitätsklinikums Göttingen mit 97,46 Euro angibt, hinzugerechnet. Somit ergeben sich pro Pflegetag und Patient Fixkosten infolge Personal - und Overheadkosten von insgesamt etwa 691,29 Euro.

\subsection{Die Berechnung der verursachten Kosten}

Zur Berechnung der durch Ressourcenverbrauch und erbrachte Leistungen verursachten Kosten wurden die erhobenen Daten - in Abstimmung mit der Leitung der Abteilung für Anaesthesiologie sowie der Abteilung für Kardiologie und Pneumologie des Universitätsklinikums Göttingen - der Fa. HealthEcon AG in Basel übersandt, die für die gesamte Multicenterstudie als Datentreuhänder fungierte. Seitens der Abteilung für Anaesthesiologie und der Zentralapotheke des Universitätsklinikums Göttingen wurden der Fa. HealthEcon AG Datenbanken mit den krankenhauseigenen Preisen und Tarifen für die einzelnen Ressourcen und Leistungen zur Verfügung gestellt.

Aufgrund dieser Datenbanken konnten sämtlichen dokumentierten Daten entsprechende Geldwerte zugeordnet werden. Unter Berücksichtigung unserer Berechnungen der Personal - und Overheadkosten des Universitätsklinikums Göttingen wurden somit die gesamten Kosten der Intensivbehandlung der betreffenden Patienten aus der Sicht des Krankenhauses für diese Studie ermittelt. 


\subsection{Die Einteilung der Patienten in LOS - Gruppen und die Auswahl der Musterpatienten A - D}

Zunächst wurden die 100 Patienten dieser Studie in LOS - Gruppen eingeteilt. In die erste Gruppe wurden Patienten mit einer LOS von unter 5 Tagen aufgenommen. Die zweite Gruppe beinhaltete Patienten mit einer LOS zwischen 5 und 10 Tagen. Die sog. „Langlieger" mit einer LOS von über 10 Tagen wurden in der dritten Gruppe betrachtet.

Um die entstandenen Kosten in ihren verschiedenen Kostenblöcken grafisch darzustellen, wurden aus den verschiedenen LOS - Gruppen jeweils ein bzw. zwei Musterpatienten (A - D) ausgewählt. Bei der Wahl der Musterpatienten wurde darauf geachtet, einen für die jeweilige LOS -Gruppe möglichst repräsentativen Patienten zu zeigen.

\subsection{Die statistische Vorgehensweise}

Die erhobenen Daten wurden nach eingehender Beratung und in enger Zusammenarbeit mit der Abteilung für Medizinische Statistik der Universität Göttingen ausgewertet und graphisch dargestellt. Zur Abbildung der Angaben über die Zusammensetzung und Struktur des Patientenkollektives wurde die Darstellung in sog. Histogrammen und Boxplots gewählt.

Des Weiteren wurden die Korrelationskoeffizienten zwischen Scores und Kosten ermittelt und mit Funktionen von Regressionsgeraden dargestellt. Als unabhängige Variable befanden sich die Scorewerte auf der $\mathrm{x}$ - Achse und waren Ausgang der Untersuchung. Als abhängige Variable wurden die Kosten auf der y - Achse aufgetragen, um festzustellen, wie diese mit der Höhe der Scorewerte steigen.

Mittels des Computerprogramms Statistika wurde die lineare Regression zwischen den Kosten und den Scorewerten berechnet und auf Signifikanz überprüft. Ein $p$ - Wert $<0,01$ galt als Nachweis für eine statistisch signifikante Korrelation. 


\section{Ergebnisse}

Das Patientenkollektiv in dieser Studie umfasst insgesamt 100 Patientinnen und Patienten, davon 29 Frauen und 71 Männer. Zunächst erfolgte die Untersuchung des Patientenguts hinsichtlich der Verteilung der Diagnosen, des Alters, der Liegedauer $($ LOS = Length of Stay), Patientengesamtkosten sowie Diagnostik -, Labor -, Medikamenten -, Overhead - und Personalkosten.

Darauf folgend wurden die Patientengesamtkosten mit den Scores und der LOS korreliert. Schließlich wurde die Verteilung der Personal -, Diagnostik -, Labor - und Medikamentenkosten anhand von Musterpatienten grafisch dargestellt.

\subsection{Deskriptive Statistik des Patientenkollektives}

\subsubsection{Die Verteilung der Diagnosen im Patientenkollektiv}

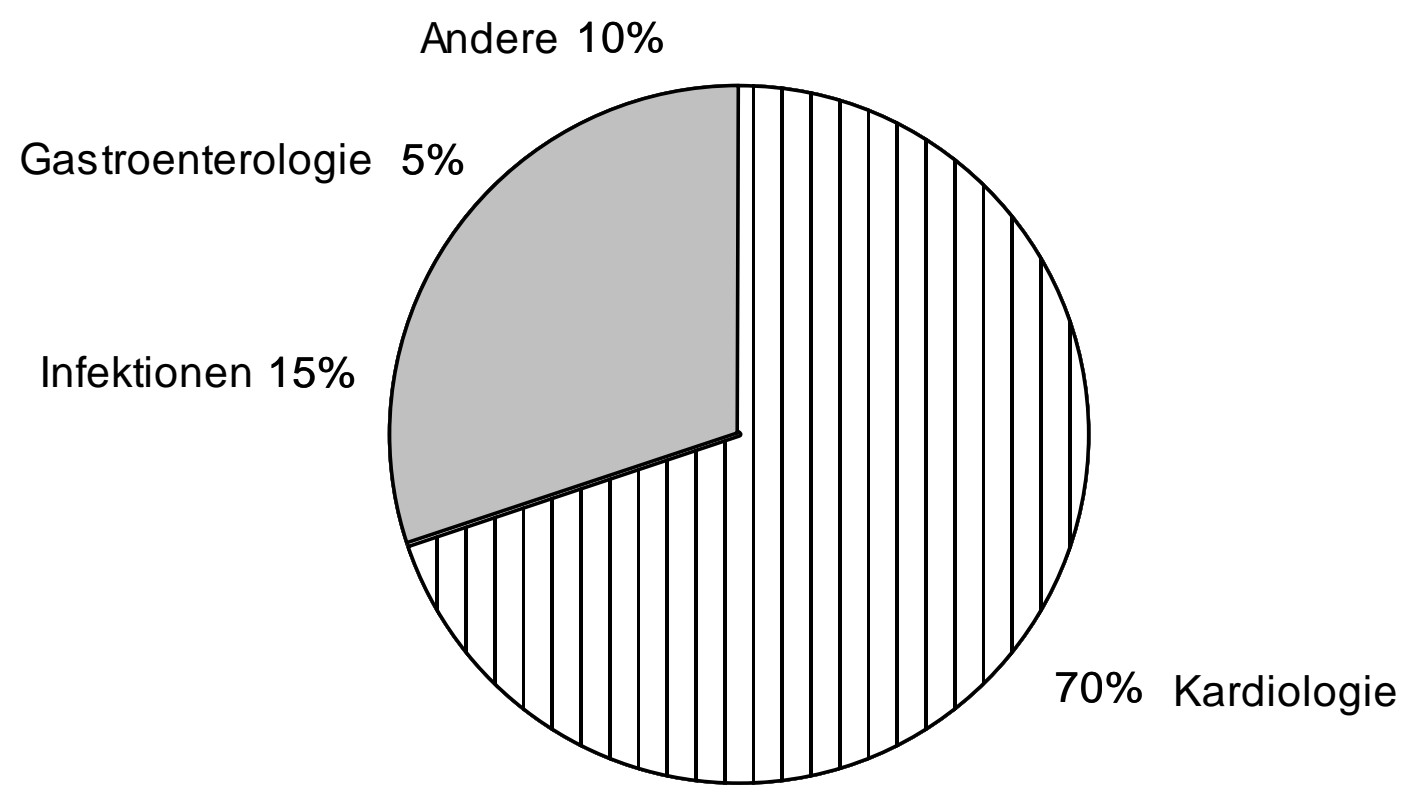

Abbildung 13: Die Diagnosenverteilung im Patientenkollektiv. 
Die Zuteilung der Patienten zu einer Diagnosengruppe erfolgte anhand ihrer ICD - 10 Hauptdiagnose. Die Verteilung der Diagnosen im Patientenkollektiv stellt sich wie folgt dar:

$70 \%$ der Patienten in dieser Studie hatten eine kardiologische Hauptdiagnose, $15 \%$ waren infektiologische und $5 \%$ gastroenterologische Fälle. Die restlichen 10\% waren Patienten mit anderen internistischen Krankheitsbildern.

\subsubsection{Die Verteilung des Patientenalters}

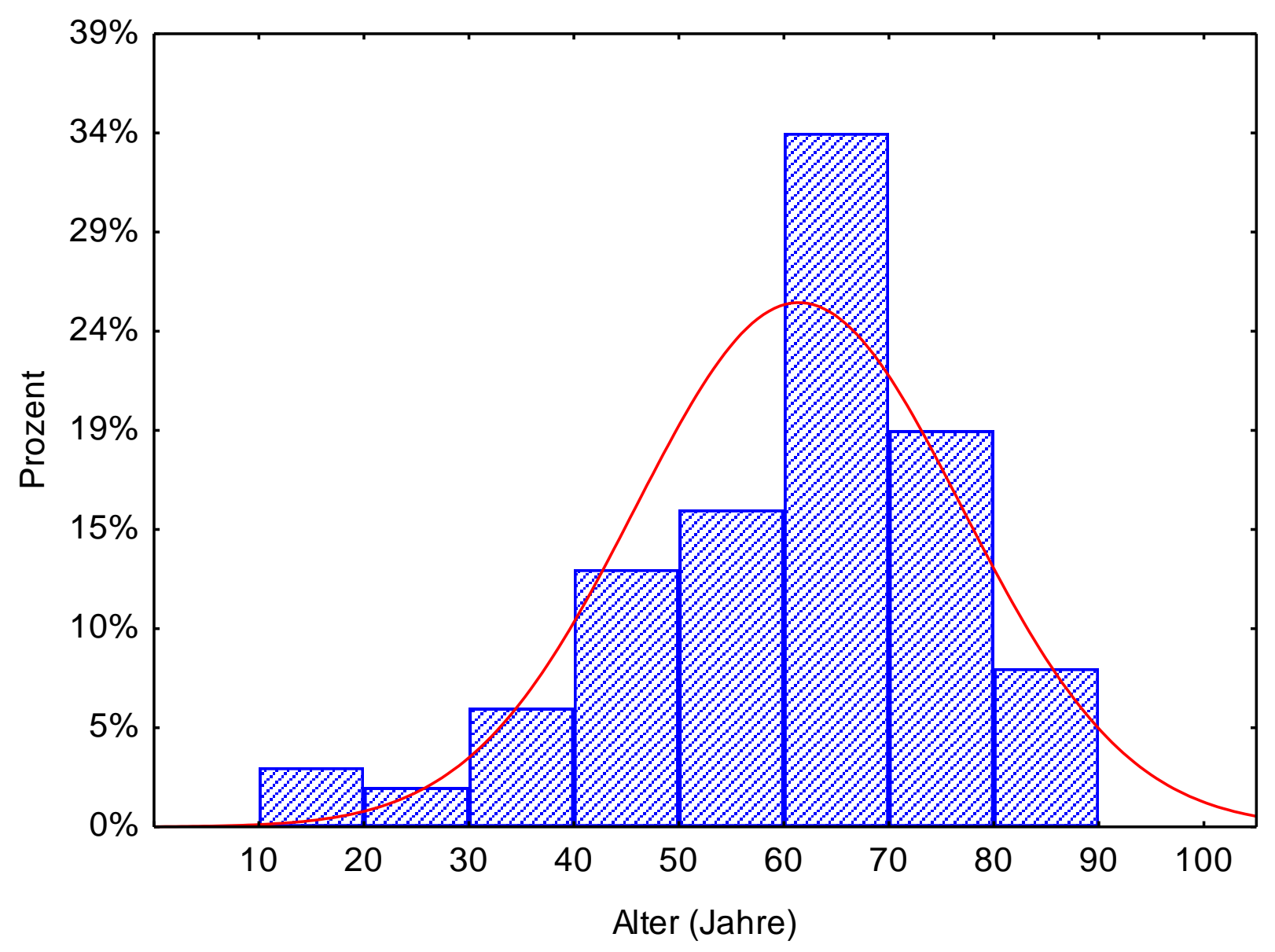

Abbildung 14: Die Altersverteilung des Patientenkollektives, angegeben in Jahren. 
Die Bandbreite des Alters der Patienten, welche in dieser Studie betrachtet wurden, erstreckte sich von 18 bis 85 Jahren. Der Altersmittelwert betrug 61,5 Jahre und der Median 65 Jahre. Für die Standardabweichung ergab sich ein Wert von 15,1 Jahren.

\subsubsection{Die Verteilung der Patientenliegedauer}

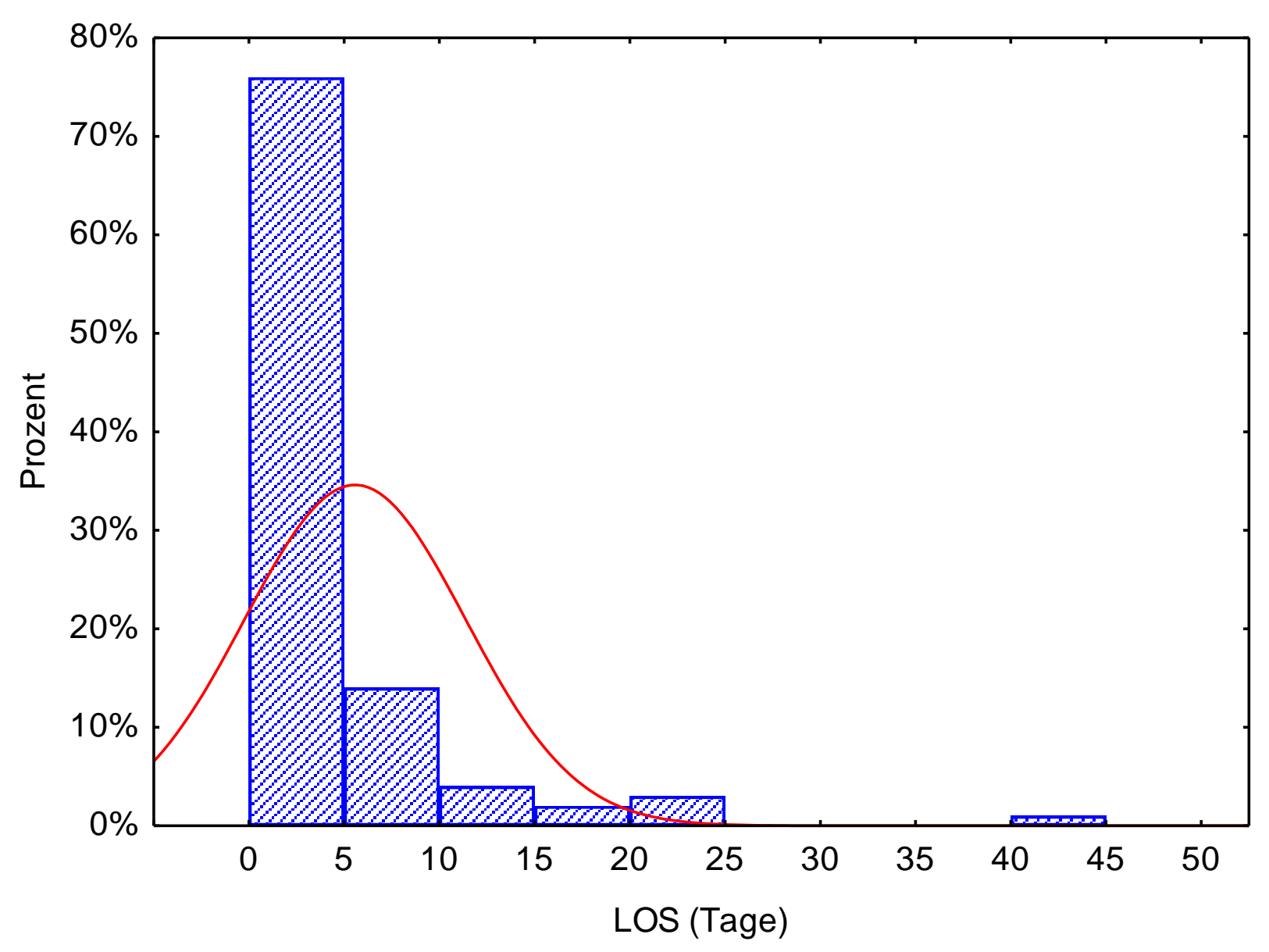

Abbildung 15: Die Verteilung der Patientenliegedauer (LOS), angegeben in Tagen.

Die individuelle intensivstationäre Patientenliegedauer (LOS) reichte in dem betrachteten Patientenkollektiv von 2 bis 42 Tage. Die durchschnittliche Patientenliegedauer betrug 5,5 Tage und der Median 4 Tage.

Die Gesamtzahl aller intensivstationären Behandlungstage in dieser Studie errechnet sich als Summe aus den jeweiligen Patientenliegedauern 
aller 100 Patienten und betrug 552 Tage. Für die Standardabweichung ergab sich ein Wert von 5,8 Tagen.

\subsubsection{Die Verteilung der Patientengesamtkosten}

Die Gesamtkosten pro Patient (inklusive Personalkosten und Overhead) für die Zeit der intensivmedizinischen Behandlung lagen im kompletten Patientenkollektiv zwischen 1.490,13 Euro und 46.408,53 Euro. Der Mittelwert der Patientengesamtkosten betrug 6.603,18 Euro, der Median 3.464,87 Euro und die Standardabweichung 7.748,47 Euro.

Die Gesamtkosten aller Patienten (inklusive Personalkosten und Overhead) in dieser Studie betrugen 660.317,69 Euro. Das ergibt für jeden Behandlungsfall einen durchschnittlichen Tageskostenbetrag von 1.196,23 Euro.

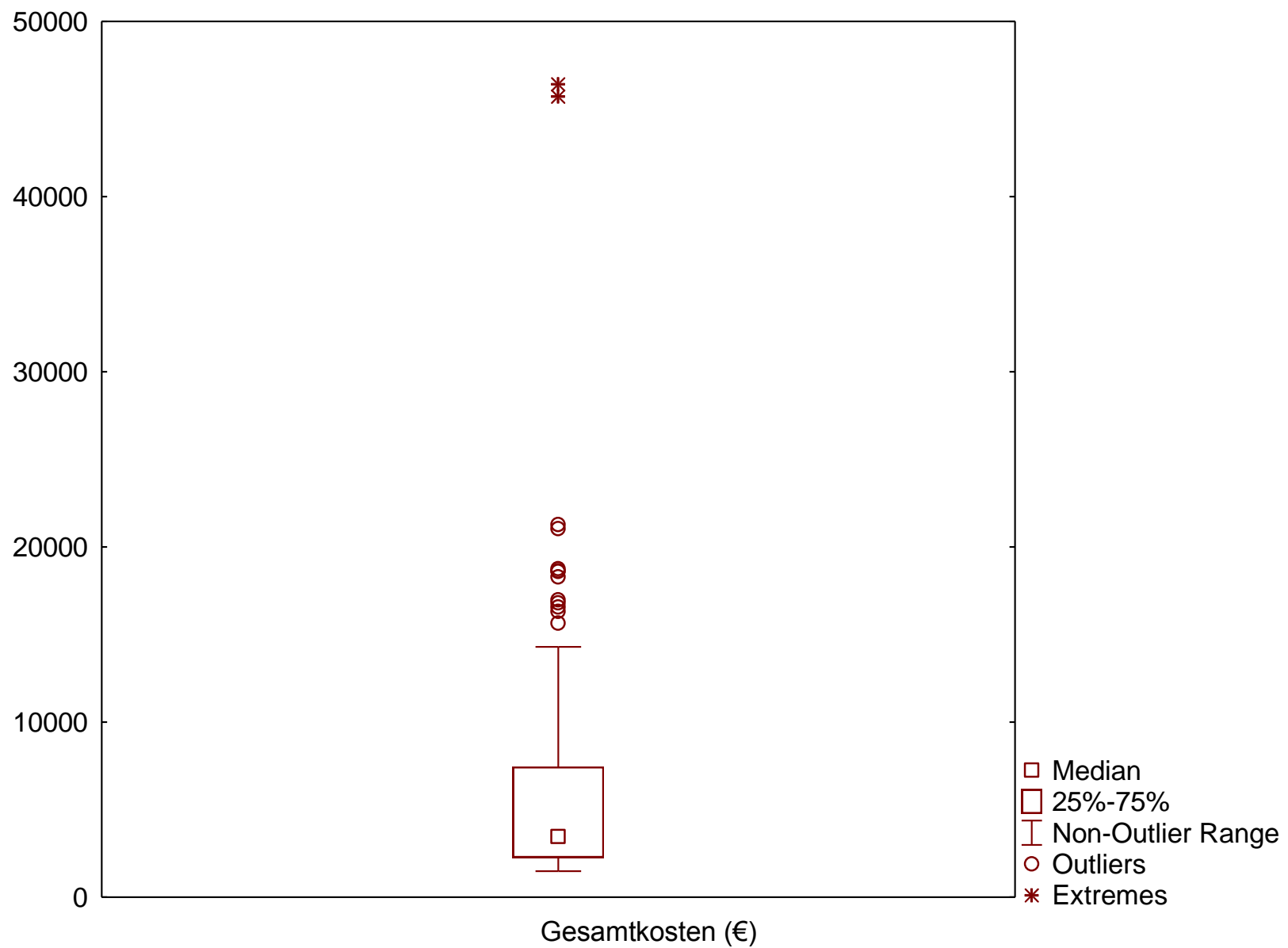

Abbildung 16: Die Verteilung der Patientengesamtkosten, angegeben in Euro. 


\subsubsection{Die Verteilung der Diagnostik -, Labor -, Medikamenten -, Overhead - und Personalkosten als Anteil der Gesamtkosten}

Im Folgenden wurden die gesamten Diagnostik -, Labor -, Medikamenten -, Overhead - und Personalkosten, die während der intensivmedizinischen Behandlung pro Patient anfielen, differenziert betrachtet. Sie bilden u. a. einen Teil der Patientengesamtkosten.

Die einzelnen Kostenblöcke haben folgende Anteile an den Patientengesamtkosten:

2\% der Gesamtkosten in dieser Studie wurden durch die Diagnostikkosten, 5\% durch die Laborkosten und 29\% durch die Medikamentenkosten verursacht. Die restlichen 64\% der Gesamtkosten entfielen auf andere Kostenblöcke. Den größten Anteil davon bildeten mit $57,8 \%$ die Personal - und Overheadkosten (siehe Kapitel 3.1.5.).

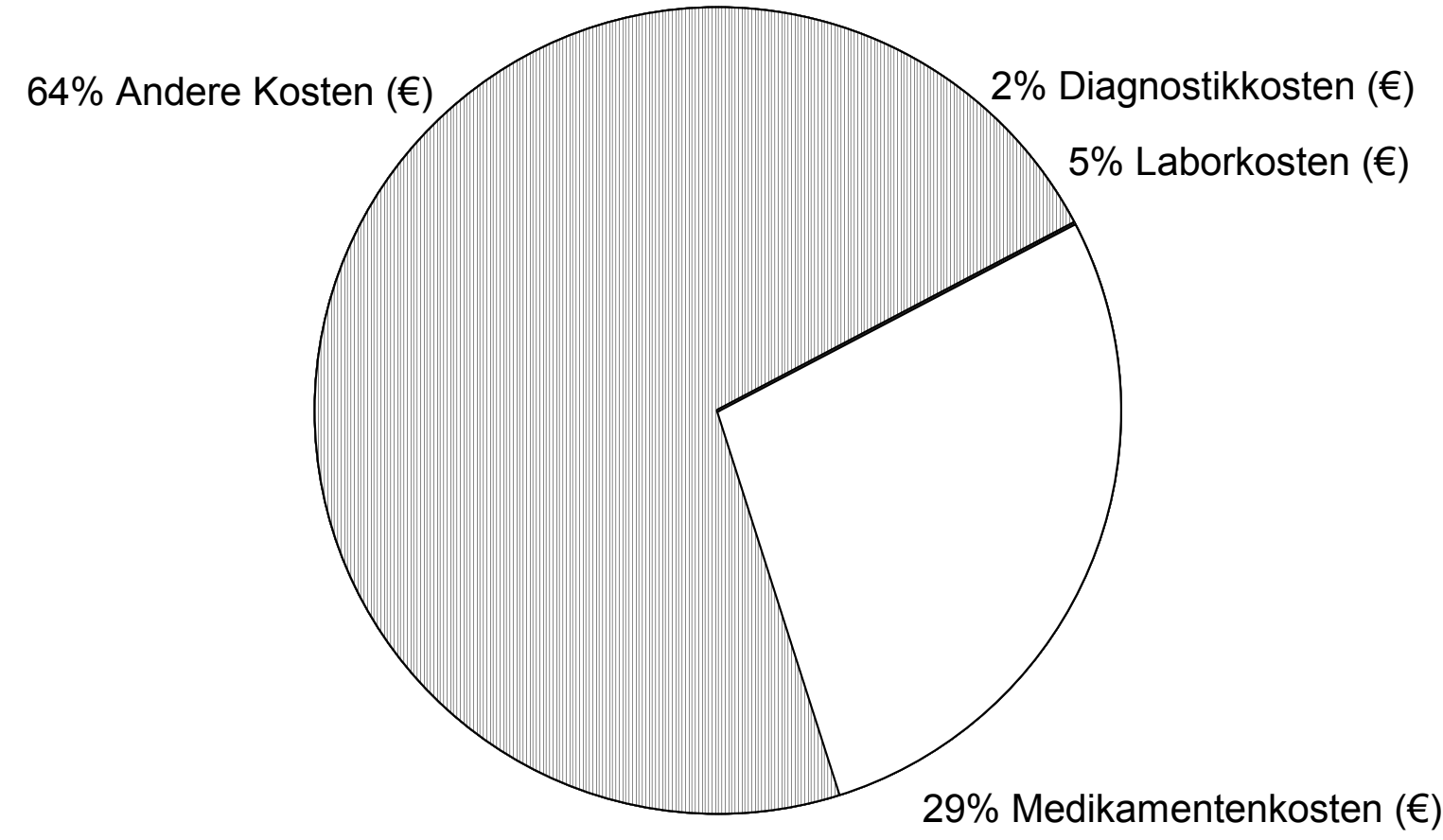

Abbildung 17: Die Kostenverteilung im Patientenkollektiv. 
In der Abbildung wurden jeweils die Mittelwerte der einzelnen Kostenblöcke, gerundet bis auf zwei Kommastellen, dargestellt.

Da sich in einem Tortendiagramm lediglich die Mittelwerte der Kosten abbilden lassen, wurden im Folgenden die einzelnen Kostenblöcke noch einmal genauer in Form eines Box Plots grafisch dargestellt.

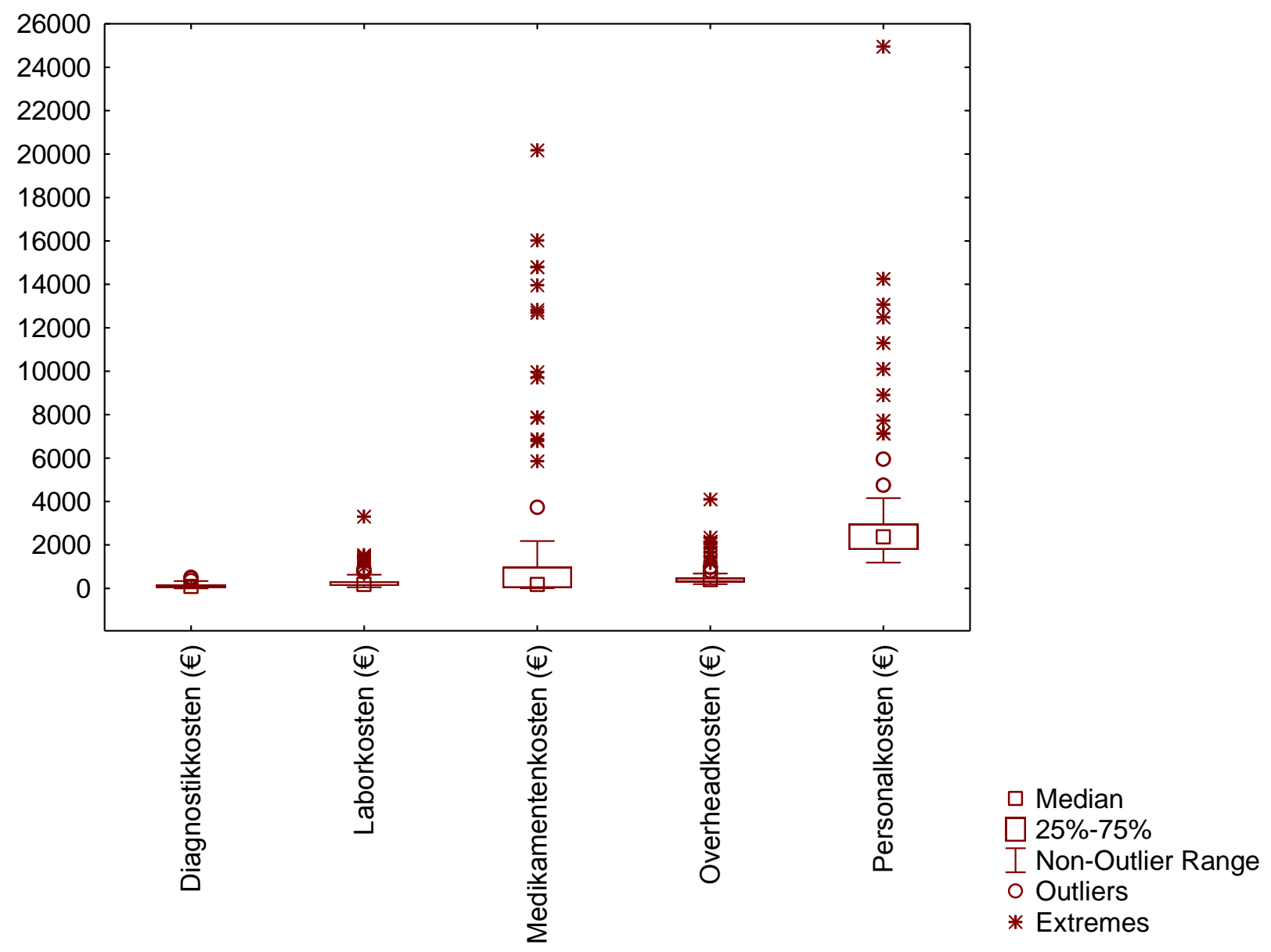

Abbildung 18: Die Verteilung der Diagnostik -, Labor -, Medikamenten -, Overhead -, und Personalkosten als Anteil der Gesamtkosten, angegeben in Euro. 
Die Diagnostikkosten setzen sich aus allen während der intensivmedizinischen Behandlung angefallenen Kosten diagnostischer Untersuchungen, wie beispielsweise EKG, röntgenologische, computertomographische, sonographische oder endoskopische Untersuchungen (siehe Kapitel 2.3.1.8.), zusammen.

Die Gesamtdiagnostikkosten pro Patient lagen im Patientenkollektiv zwischen 6,98 Euro und 496,13 Euro. Der Mittelwert betrug 116,74 Euro pro Patient und der Median 80,34 Euro. Für die Standardabweichung errechnete sich ein Wert von 110,17 Euro.

Die Summe der Gesamtdiagnostikkosten, welche in dieser Studie entstanden, betrug 11.557,61 Euro. Das waren 1,75\% der Gesamtkosten.

Die Laborkosten bestehen aus sämtlichen in dieser Studie anfallenden Kosten aus labormedizinischen und mikrobiologischen Untersuchungen (siehe Kapitel 2.3.1.9. und 2.3.1.10). Ferner kommen Kosten aus Spezialuntersuchungen im labormedizinischen bzw. mikrobiologischen Bereich hinzu.

Die Laborkosten pro Patient lagen in einem Intervall von 51,86 Euro bis 3.292,59 Euro. Der Mittelwert betrug 328,47 Euro, der Median 174,25 Euro und die Standardabweichung 436,57 Euro.

Die Summe der gesamten Laborkosten dieser Studie betrug 32.846,54 Euro. Das waren 4,98\% der Gesamtkosten.

Die Medikamentenkosten setzen sich aus allen während der intensivmedizinischen Behandlung angefallenen Kosten für Medikation im engeren Sinne, Perfusortherapie, Volumenersatz und Basisinfusionen sowie Ernährung und Supplemente (siehe Kapitel 2.3.1.1., Kapitel 2.3.1.2., Kapitel 2.3.1.4. und Kapitel 2.3.1.5.) zusammen.

Die Medikamentenkosten erstreckten sich von 2,69 Euro bis 20.179,71 Euro. Der Mittelwert betrug 1.933,01 Euro, der Median 181,37 Euro und die Standardabweichung 4.202,76 Euro.

Für die gesamten Medikamentenkosten in dieser Studie ergab sich eine Summe von 193.300,71 Euro. Das waren 29,3\% der Gesamtkosten. 
In dieser Studie wurden bei 100 Patienten insgesamt 552 Behandlungstage dokumentiert. Da die täglichen Personal - und Overheadkosten pro Patient bei 691,29 Euro liegen (siehe Kapitel 2.3.3.), ergeben sich Personalkosten von insgesamt 381.592,08 Euro. Bei Gesamtkosten von 660.317,69 Euro in dieser Studie liegt der Anteil der Personalkosten somit bei $57,8 \%$.

\subsection{Die Korrelationen zwischen Scores und Kosten}

\subsubsection{Die Korrelationen zwischen Gesamtscores und Gesamtkosten}

\subsubsection{Die Korrelation zwischen dem Gesamt - SAPS II und den Gesamtkosten}

Unter der Hypothese, dass mit der Höhe der Scorewerte auch die Kosten steigen würden, wurde die Abhängigkeit zwischen den individuellen gesamten SAPS II - Scorepunkten (Summe aller täglichen SAPS II - Werte) und den individuellen Gesamtkosten geprüft. Die Punkte in allen folgenden Abbildungen entsprechen den numerischen Werten der ermittelten Daten dieser Studie. 


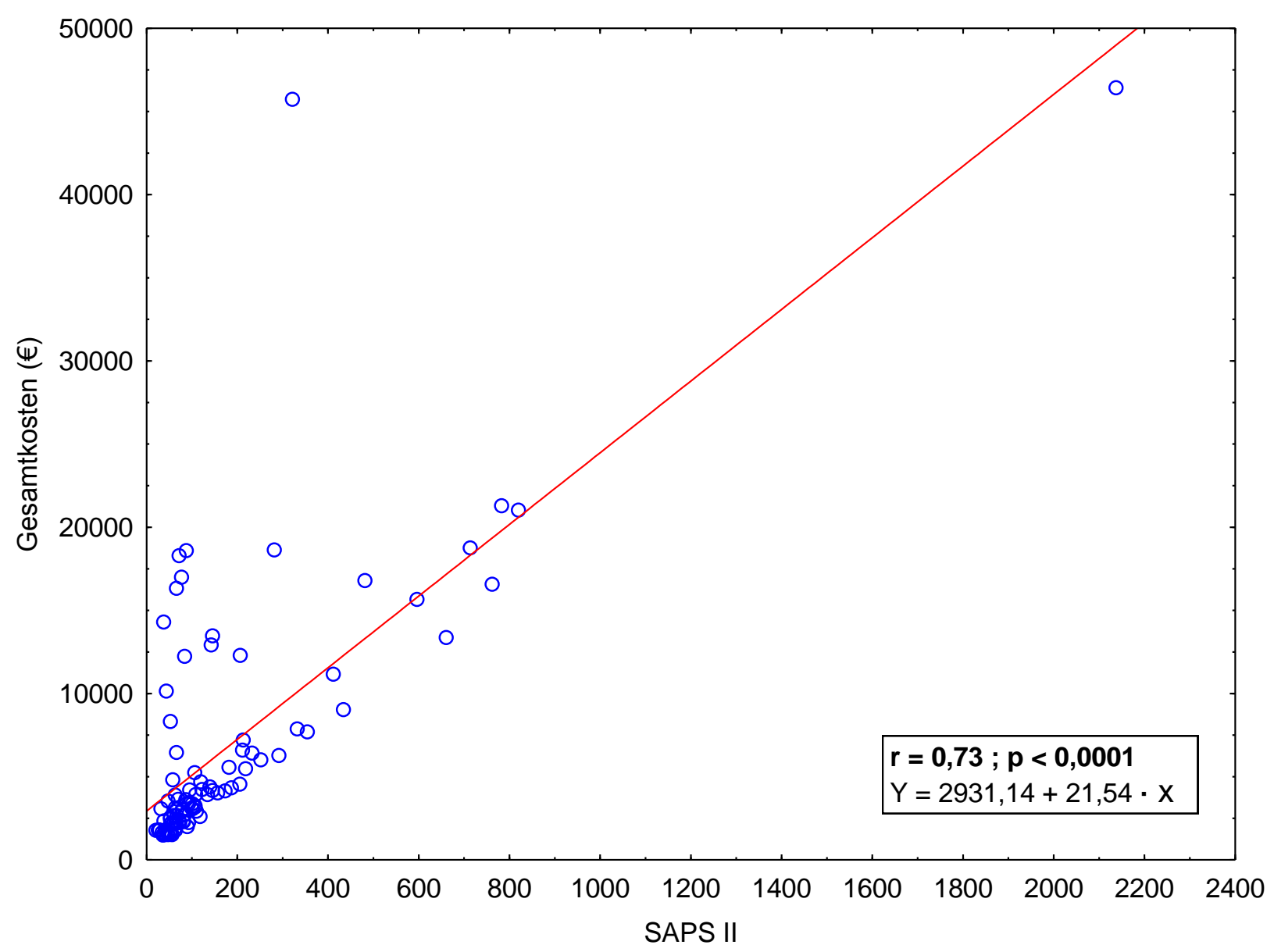

Abbildung 19: Die Korrelation zwischen dem Gesamt - SAPS II und den Gesamtkosten.

Für die Korrelation zwischen den Gesamt - SAPS II - Scorepunkten und den Gesamtkosten im ganzen Patientenkollektiv ergibt sich ein Korrelationskoeffizient von 0,73 . Demnach besteht eine Korrelation zwischen beiden betrachteten Variablen.

Die Funktion der Regressionsgeraden, welche errechnet wurde, lautet:

$$
Y=2931,14+21,54 \cdot X
$$

Diese Funktion ergibt den Graphen, dem die erhobenen Messdaten am nächsten anliegen. 


\subsubsection{Die Korrelation zwischen dem Gesamt - TISS - 28 und den Gesamtkosten}

Die Hypothese für die Prüfung der Abhängigkeit zwischen Kosten und TISS - 28 lautet ebenso wie für den SAPS II, dass die Kosten mit den Scores steigen müssten.

Ausgehend von der Annahme, dass die größte Abhängigkeit zwischen Scores und Kosten beim Leistungsscore TISS - 28 besteht, ergibt sich für das gesamte Patientenkollektiv tatsächlich ein hoher Korrelationskoeffizient von 0,75.

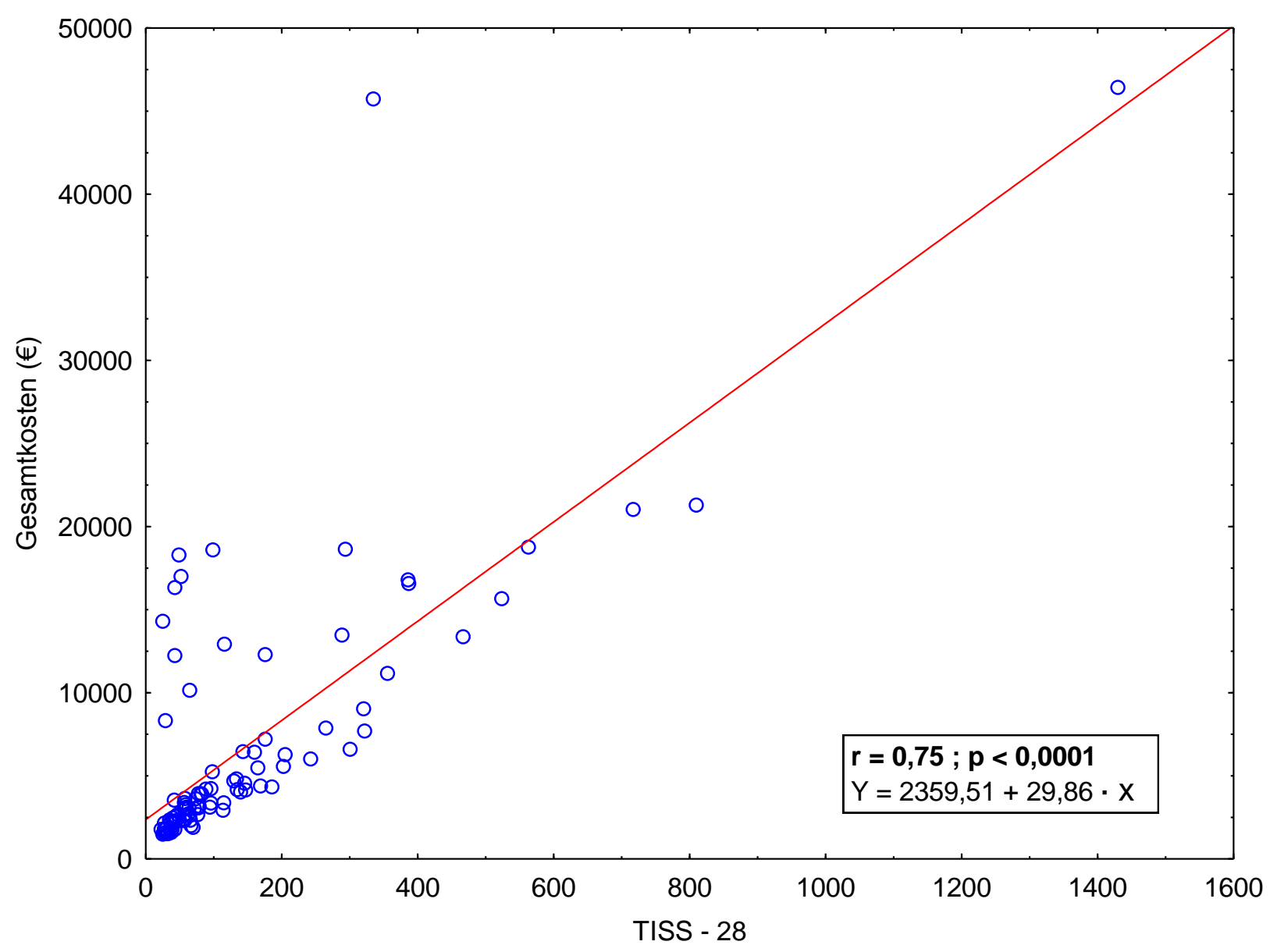

Abbildung 20: Die Korrelation zwischen dem Gesamt - TISS - 28 und den Gesamtkosten. 
Die Funktion der Regressionsgeraden lautet:

$$
Y=2359,51+29,86 \cdot X
$$

Die Funktion erlaubt die Prognose der Gesamtkosten, welche sich aus einem bestimmten Scorewert ergeben.

\subsubsection{Die Korrelation zwischen dem Gesamt - SAPS II bzw. dem Gesamt - TISS - 28 und den gesamten Diagnostik -, Labor - und Medikamentenkosten als Anteil der Gesamtkosten}

Im Folgenden wurden jeweils die Diagnostik -, Labor - und Medikamentenkosten, unter der Hypothese einer bestehenden Korrelation zwischen ihnen und den Gesamt - SAPS II - bzw. den Gesamt - TISS - 28 - Scorewerten, untersucht.

Dabei ergab sich die jeweils deutlichste Korrelation zwischen den Laborkosten und den Gesamtscorewerten sowohl für den SAPS II, als auch für den TISS - 28. Für die Medikamentenkosten konnte keine Korrelation zwischen ihnen und den Gesamtscorewerten des SAPS II bzw. des TISS - 28 nachgewiesen werden.

\begin{tabular}{|l|c|c|}
\hline & SAPS II & TISS -28 \\
\hline Diagnostikkosten & $r=0,67 ; p<0,0001$ & $r=0,73 ; p<0,0001$ \\
\hline Laborkosten & $r=0,94 ; p<0,0001$ & $r=0,97 ; p<0,0001$ \\
\hline Medikamentenkosten & $r=0,18 ; p=0,066$ & $r=0,19 ; p=0,055$ \\
\hline
\end{tabular}

Tabelle 3: Die Korrelationskoeffizienten zwischen dem Gesamt - SAPS II bzw. dem Gesamt - TISS - 28 und den gesamten Diagnostik -, Labor -, und Medikamentenkosten. 


\subsubsection{Die Korrelation zwischen der Patientenliegedauer (LOS) und den Gesamtkosten}

Im Weiteren wurde unter der Hypothese, dass mit der Anzahl der LOS - Tage auch die Gesamtkosten (inklusive Personalkosten und Overhead) steigen würden, die Abhängigkeit zwischen ihnen untersucht.

Für die Korrelation zwischen der Patientenliegedauer und den Gesamtkosten beträgt der Korrelationskoeffizient 0,75. Der Aufwand der Intensivbehandlung korreliert demnach mit der Patientenliegedauer.

Die Funktion, welche sich für die errechnete Regressionsgerade ergibt, ist:

$$
Y=1062,33+1003,78 \cdot X
$$

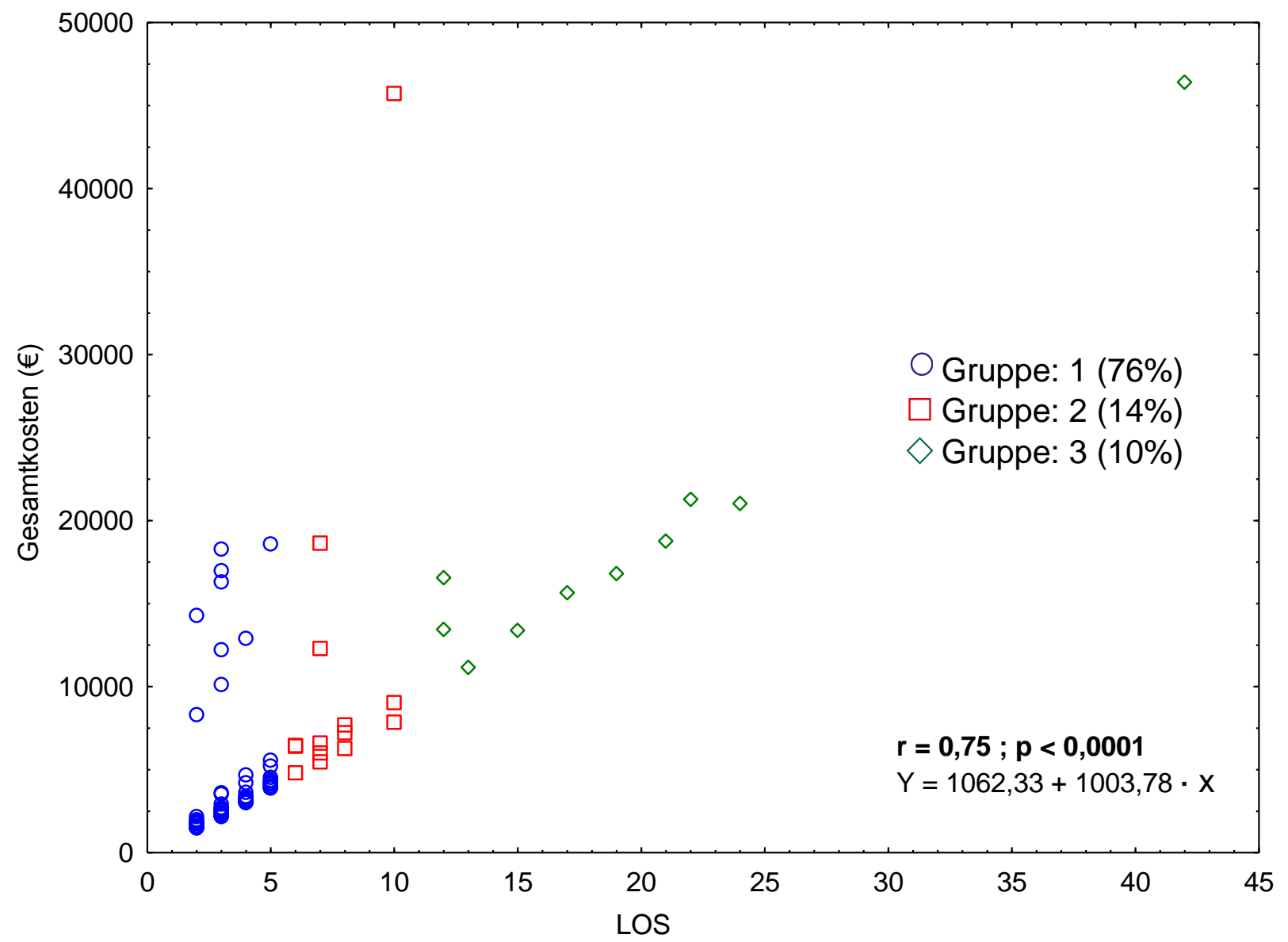

Abbildung 21: Die Korrelation zwischen der Patientenliegedauer (LOS) und den Gesamtkosten. 
Die Abbildung zeigt die Korrelation zwischen der Liegedauer und den Gesamtkosten unter Einteilung der Patienten in drei Gruppen. Die erste Gruppe beinhaltet Patienten mit einer LOS von unter 5 Tagen. Sie stellt mit $76 \%$ den größten Anteil des in der vorliegenden Arbeit untersuchten Patientengutes dar. In der zweiten Gruppe befinden sich Patienten mit einer LOS zwischen 5 und 10 Tagen. Dies waren $14 \%$ der Patienten. Die Gruppe drei beinhaltet die so genannten "Langlieger" mit einer LOS von über 10 Tagen. Sie stellt die kleinste Gruppe mit $10 \%$ der Patienten dar.

\subsubsection{Die Korrelation zwischen den Gesamtscorewerten und den} Gesamtkosten in einzelnen LOS - Patientengruppen

\subsubsection{Die Korrelation zwischen dem Gesamt - SAPS II und den Gesamtkosten bei Patienten mit einer LOS unter 5 Tagen}

Die schon gezeigte Korrelation zwischen Gesamt - SAPS II und Gesamtkosten wurde im Folgenden für die einzelnen LOS - Patientengruppen unter der Hypothese einer auch hier bestehenden Korrelation differenziert untersucht. 


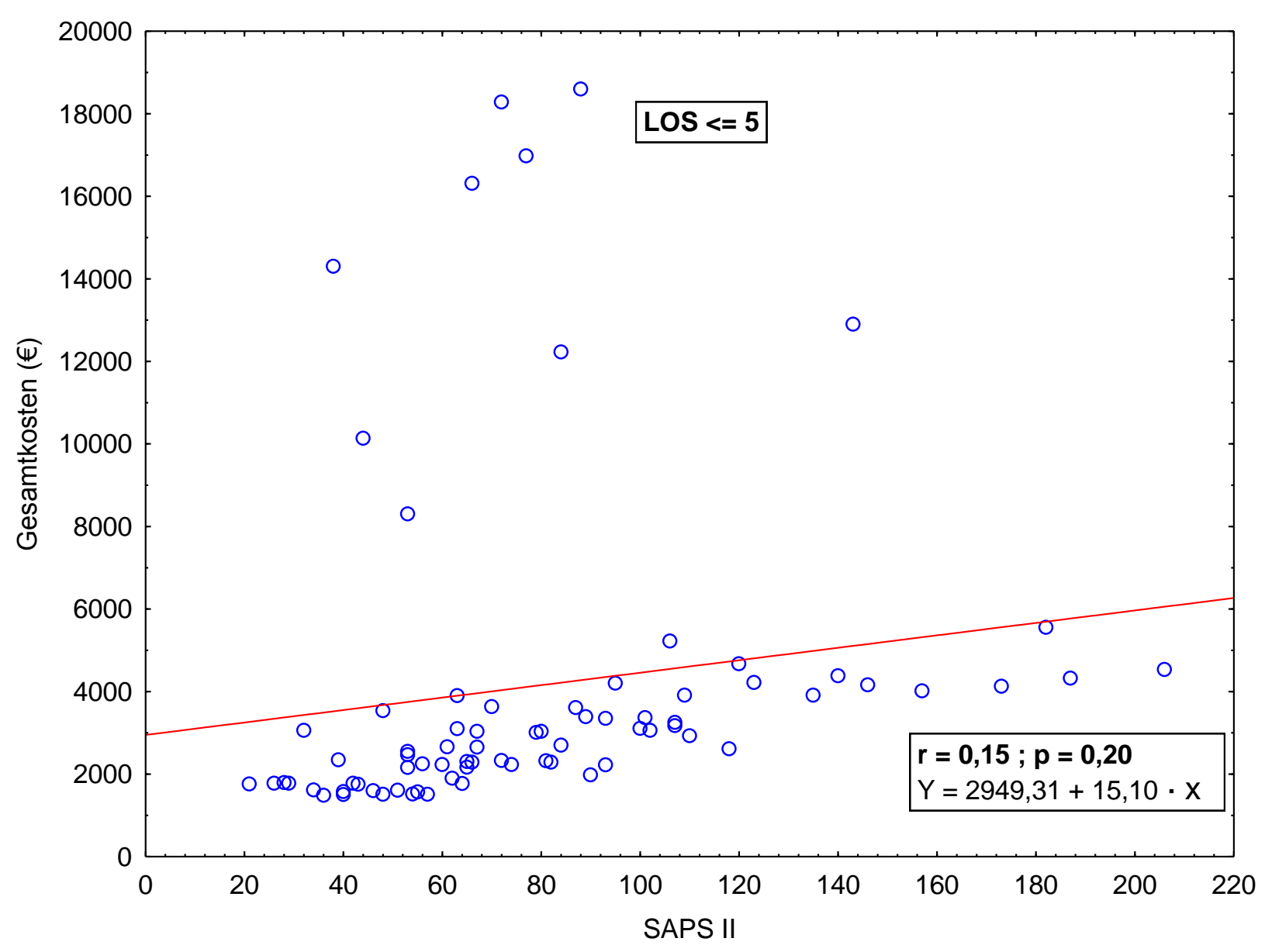

Abbildung 22: Die Korrelation zwischen dem Gesamt - SAPS II und den Gesamtkosten bei Patienten mit einer LOS $<=5$ Tage.

Der Korrelationskoeffizient zwischen den Gesamt - SAPS II - Scorewerten und den Gesamtkosten (inklusive Personalkosten und Overhead) in der ersten Patientengruppe mit einer LOS von unter 5 Tagen beträgt 0,15. Demnach besteht in dieser Patientengruppe zwischen den untersuchten Variablen keine Korrelation.

Für die Funktion der Regressionsgeraden errechnet sich folgende Formel:

$$
Y=2949,31+15,10 \cdot X
$$




\subsubsection{Die Korrelation zwischen dem Gesamt - SAPS II und den Gesamtkosten bei Patienten mit einer LOS von 5-10 Tagen}

Für die Korrelation zwischen den Gesamt - SAPS II - Scorewerten und den Gesamtkosten (inklusive Personalkosten und Overhead) für Patienten mit einer LOS zwischen 5 und 10 Tagen ergibt sich ein Korrelationskoeffizient von 0,29. Zwischen innen besteht demnach auch in der zweiten Patientengruppe keine Korrelation.

Die errechnete Regressionsgerade hat die Funktion:

$$
Y=3289,83+30,02 \cdot X
$$

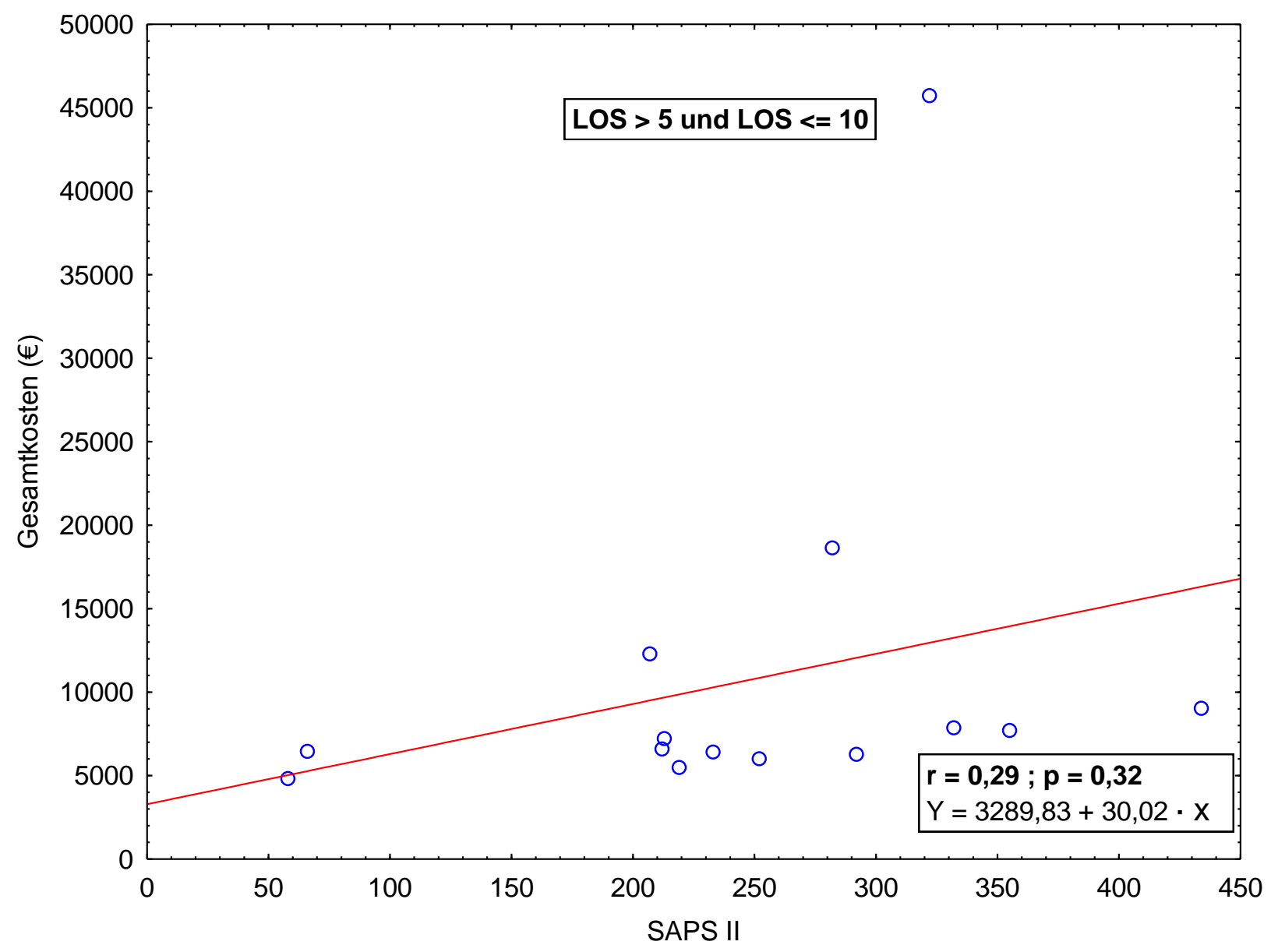

Abbildung 23: Die Korrelation zwischen dem Gesamt - SAPS II und den Gesamtkosten bei Patienten mit einer LOS $>5$ und $<=10$ Tage. 


\subsubsection{Die Korrelation zwischen dem Gesamt - SAPS II und den Gesamtkosten bei Patienten mit einer LOS von über 10 Tagen}

Der Korrelationskoeffizient zwischen den Gesamt- SAPS II - Scorewerten und den Gesamtkosten (inklusive Personalkosten und Overhead) beträgt in der Gruppe der Langlieger 0,96. Somit besteht zwischen den untersuchten Variablen in der Gruppe der Langlieger eine sehr deutliche Korrelation.

Die Funktion, welche sich für die errechnete Regressionsgerade ergibt, ist:

$$
Y=5760,10+18,22 \cdot X
$$

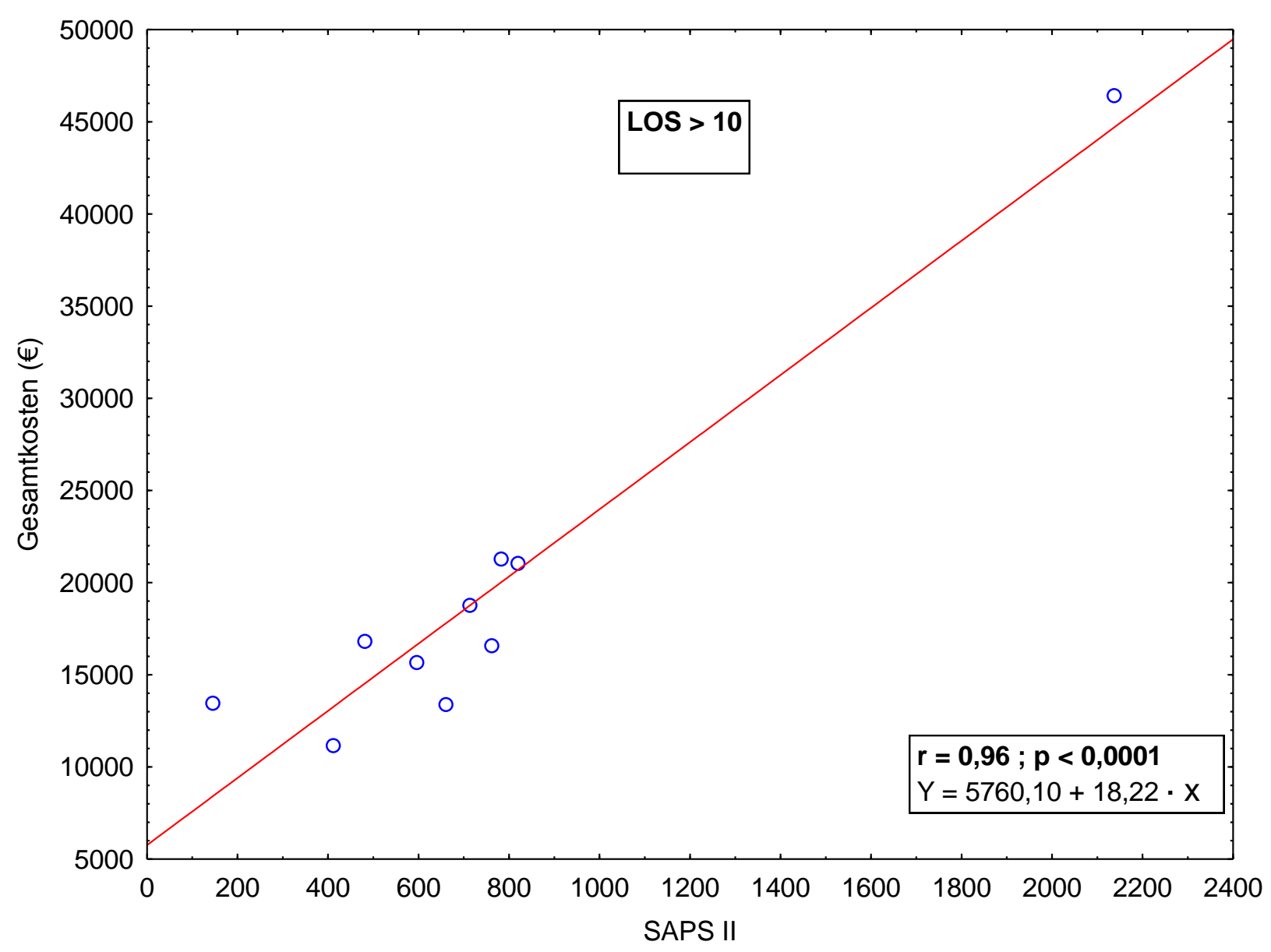

Abbildung 24: Die Korrelation zwischen dem Gesamt - SAPS II und den Gesamtkosten bei Patienten mit einer LOS > 10 Tage. 


\subsubsection{Die Korrelation zwischen dem Gesamt - TISS - 28 und den Gesamtkosten bei Patienten mit einer LOS unter 5 Tagen}

Ausgehend von der Hypothese, dass die schon gezeigte Korrelation zwischen Gesamt - TISS - 28 und Gesamtkosten sich auch in den einzelnen LOS - Patientengruppen zeigt, wurde folgend die Abhängigkeit zwischen beiden Variablen differenziert für die einzelnen Gruppen geprüft.

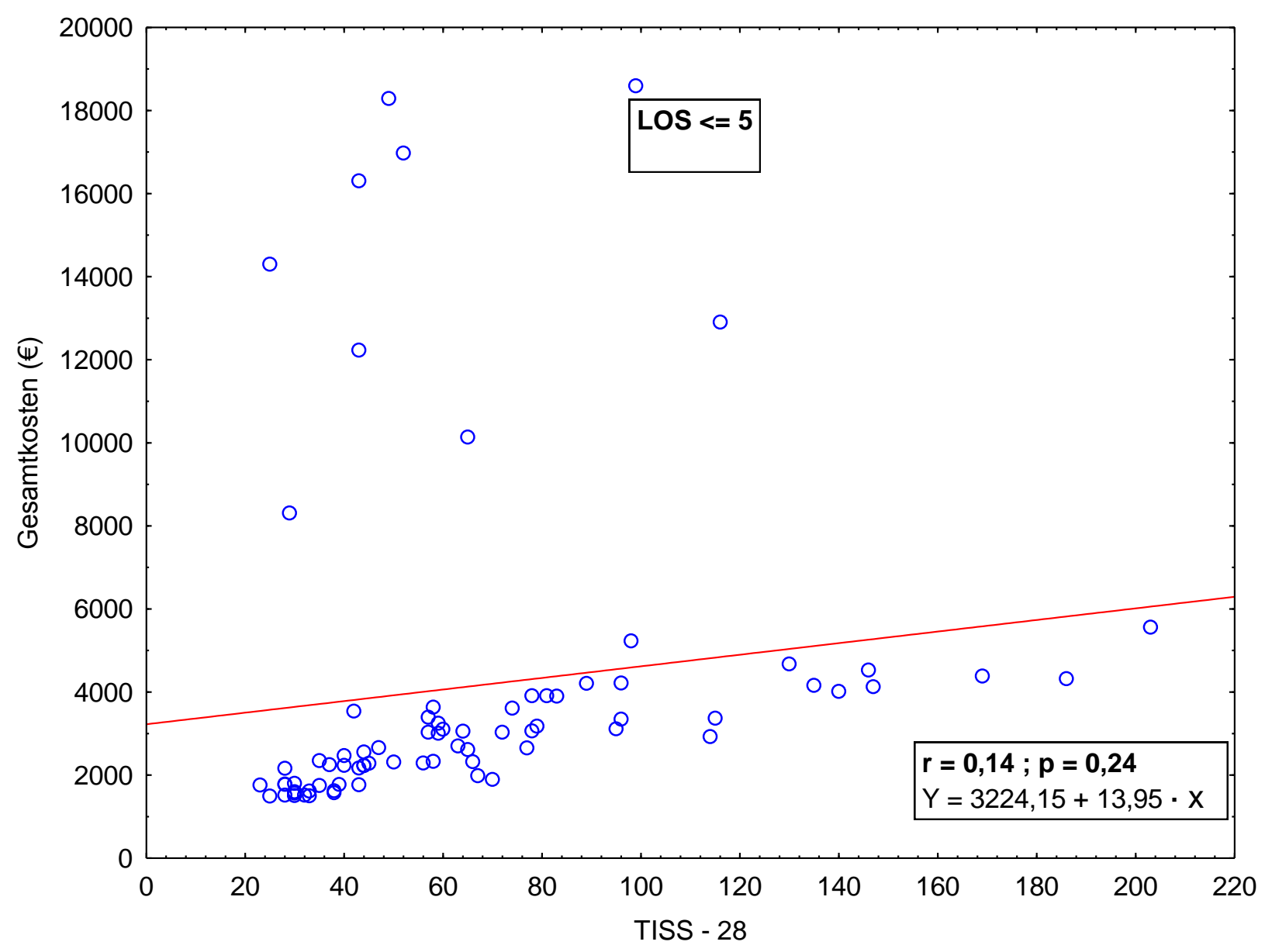

Abbildung 25: Die Korrelation zwischen dem Gesamt - TISS - 28 und den Gesamtkosten bei Patienten mit einer LOS <= 5 Tage. 
Für die Korrelation zwischen den Gesamt - TISS - 28 - Scorewerten und den Gesamtkosten (inklusive Personalkosten und Overhead) ergibt sich in der ersten Patientengruppe, in welcher Patienten mit einer LOS von unter 5 Tagen betrachtet wurden, ein Korrelationskoeffizient von 0,14. Demnach besteht keine Korrelation zwischen den untersuchten Größen in der ersten Patientengruppe.

Die errechnete Regressionsgerade hat die Funktion:

$$
Y=3224,15+13,95 \cdot X
$$




\subsubsection{Die Korrelation zwischen dem Gesamt - TISS - 28 und den Gesamtkosten bei Patienten mit einer LOS von 5-10 Tagen}

Der Korrelationskoeffizient für die Korrelation zwischen den Gesamt - TISS - 28 - Scorewerten und den Gesamtkosten (inklusive Personalkosten und Overhead) beträgt bei Patienten mit einer LOS zwischen 5 und 10 Tagen 0,5. Demnach besteht für die betrachteten Variablen in dieser Patientengruppe eine geringe Korrelation.

Für die Regressionsgerade wurde die Funktion:

$$
Y=-5935,33+72,08 \cdot X
$$

errechnet.

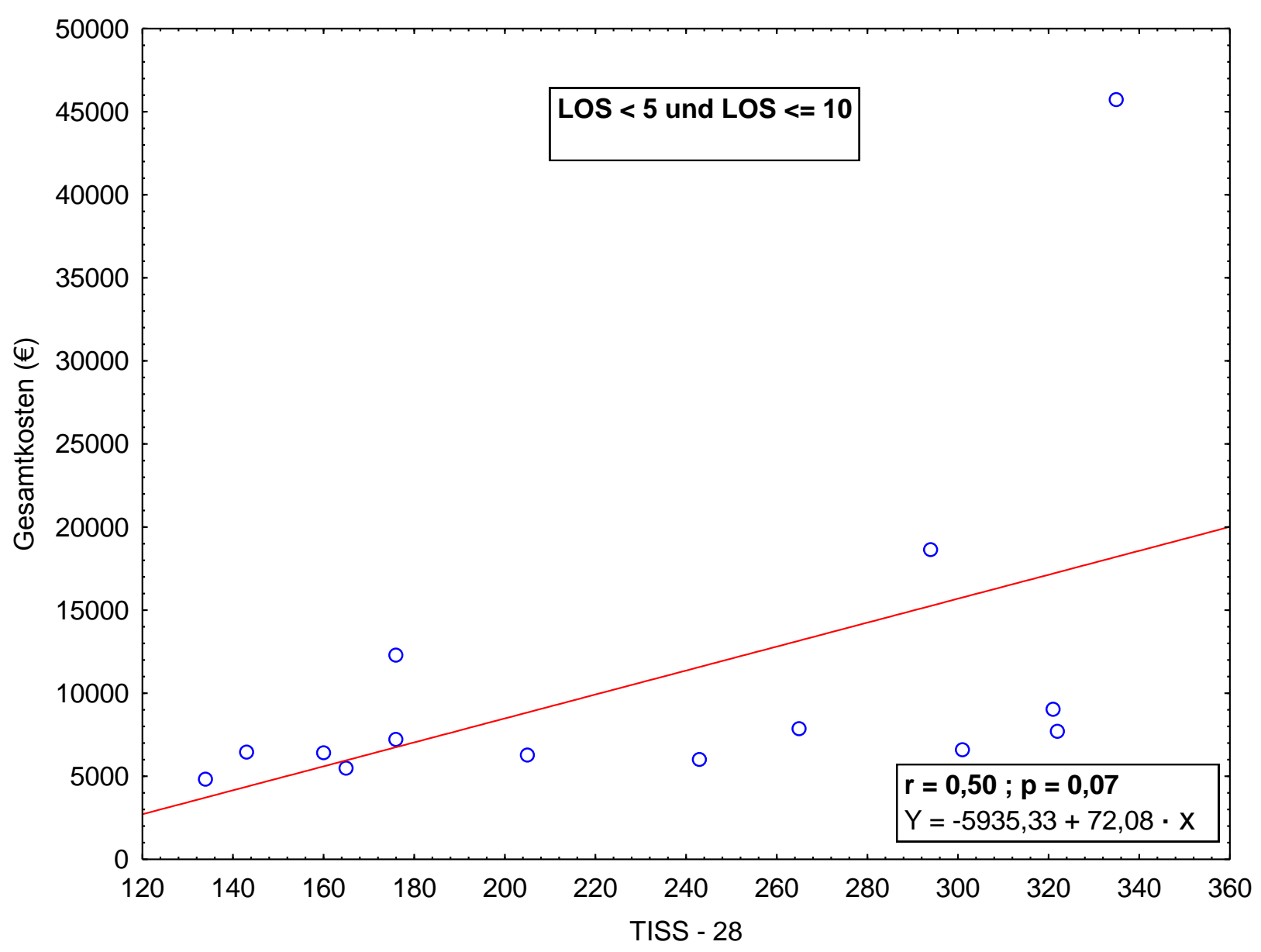

Abbildung 26: Die Korrelation zwischen dem Gesamt TISS - 28 und den Gesamtkosten bei Patienten mit einer LOS $<5$ und $<=10$ Tage. 


\subsubsection{Die Korrelation zwischen dem Gesamt - TISS - 28 und den Gesamtkosten bei Patienten mit einer LOS von über 10 Tagen}

Für die Korrelation zwischen den Gesamt - TISS - 28 - Scorewerten und den Gesamtkosten errechnet sich für die Gruppe der Langlieger ein Korrelationskoeffizient von 0,96 . Somit besteht auch hier zwischen den untersuchten Variablen in der Gruppe der Langlieger eine sehr deutliche Korrelation.

Die errechnete Regressionsgerade hat die Funktion:

$$
Y=2484,42+28,61 \cdot X
$$

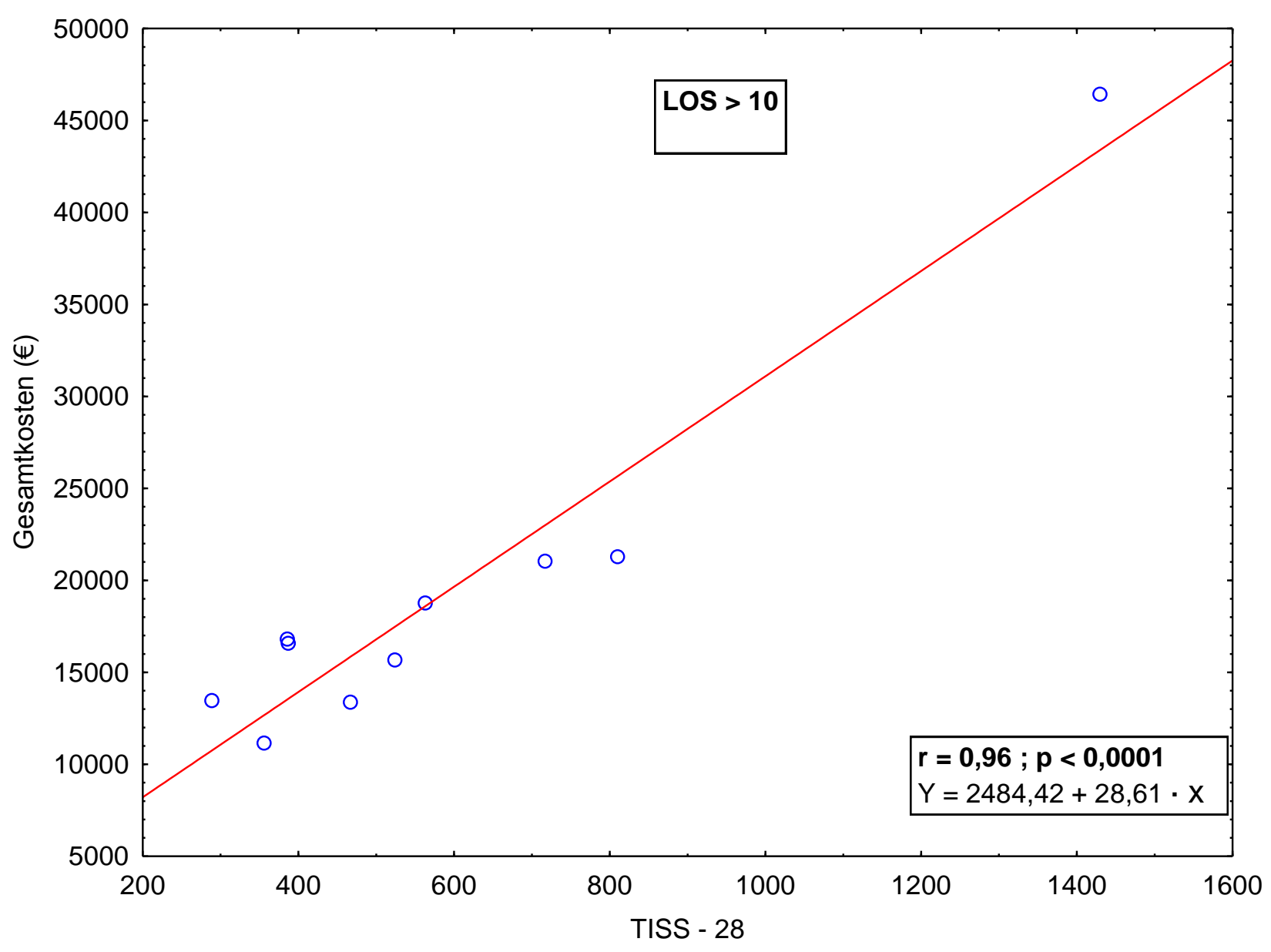

Abbildung 27: Die Korrelation zwischen dem Gesamt - TISS - 28 und den Gesamtkosten bei Patienten mit einer LOS > 10 Tage. 
3.3. Die Verteilung der Personal -, Diagnostik -, Labor - und Medikamentenkosten bei einzeln ausgewählten Musterpatienten

\subsubsection{Die Verteilung der Personal -, Diagnostik -, Labor - und} Medikamentenkosten zweier Musterpatienten ( $A$ und $B)$ mit einer LOS unter 5 Tagen

Um die unterschiedlichen Korrelationskoeffizienten in den drei verschiedenen Patientengruppen besser verstehen zu können, wurde jeweils anhand eines Musterpatienten $\boldsymbol{A}$ die Aufteilung der einzelnen Kostenblöcke betrachtet.

Die Verteilung der einzelnen Kostenblöcke bei einem Musterpatienten aus der ersten Patientengruppe, in welcher Patienten mit einer LOS von unter 5 Tagen betrachtet wurden, stellt sich wie folgt dar:

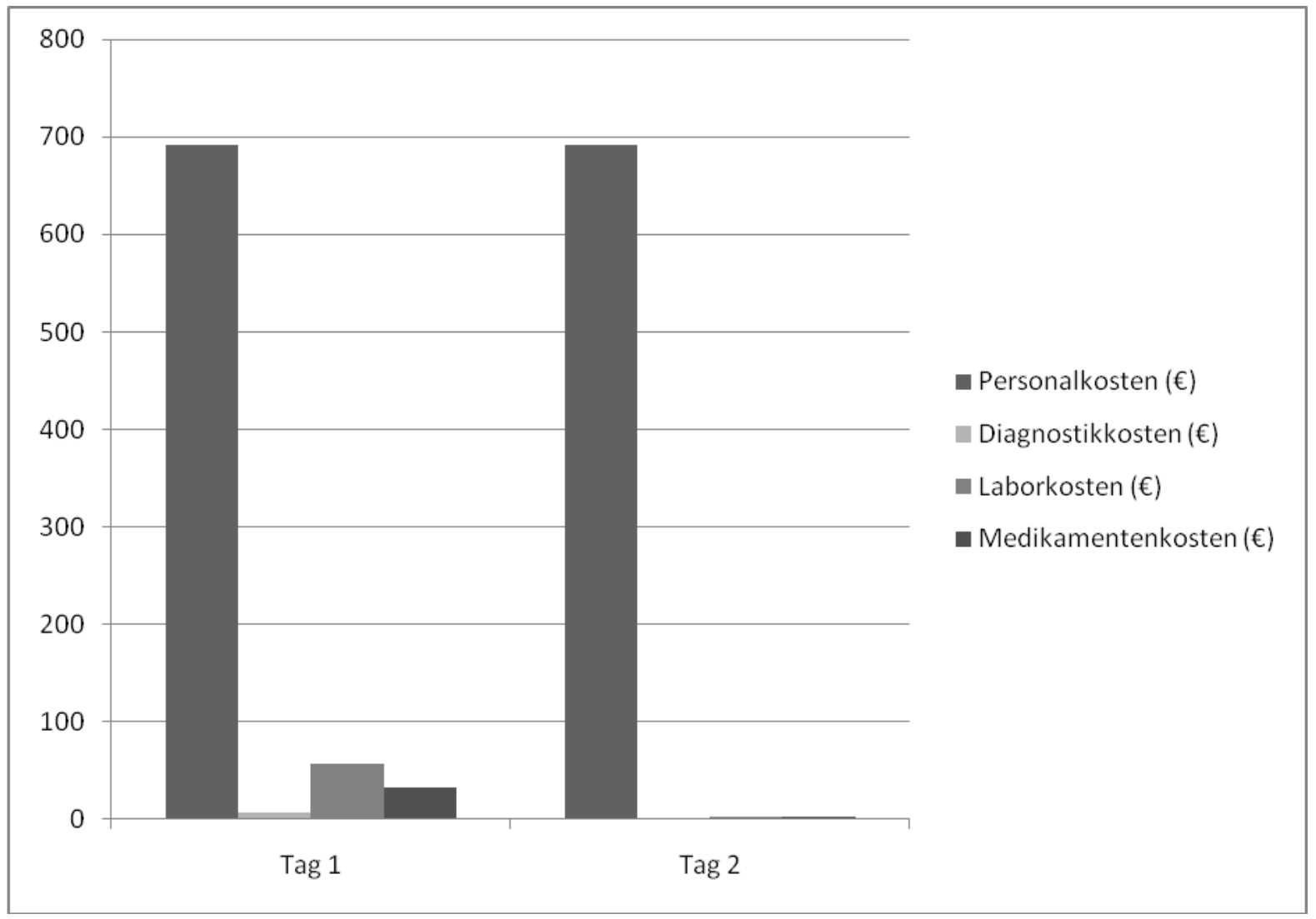

Abbildung 28: Die Verteilung der Personal -, Diagnostik -, Labor - und Medikamentenkosten des Musterpatienten A mit einer LOS von 2 Tagen, angegeben in Euro. 
Der Musterpatient $\boldsymbol{A}$ mit einer LOS von 2 Tagen verursachte Personalkosten in Höhe von 691,29 Euro pro Tag. Diagnostikkosten fielen nur am ersten Tag in Höhe von 6,98 Euro an. Die Laborkosten betrugen am ersten Tag 57,29 Euro und am zweiten Tag 2,76 Euro. Die Medikamentenkosten lagen an Tag eins bei 32,68 Euro und an Tag zwei bei 3,21 Euro.

Betrachtet man einen zweiten Musterpatienten $\boldsymbol{B}$ aus der Patientengruppe, welche Patienten mit einer LOS von unter 5 Tagen beinhaltet, stellt man eine ähnliche Kostenverteilung fest.

Der Musterpatient $\boldsymbol{B}$ hat eine LOS von 3 Tagen. Auch bei ihm bilden die Personalkosten mit 691,29 Euro pro Tag den Hauptanteil der Kosten.

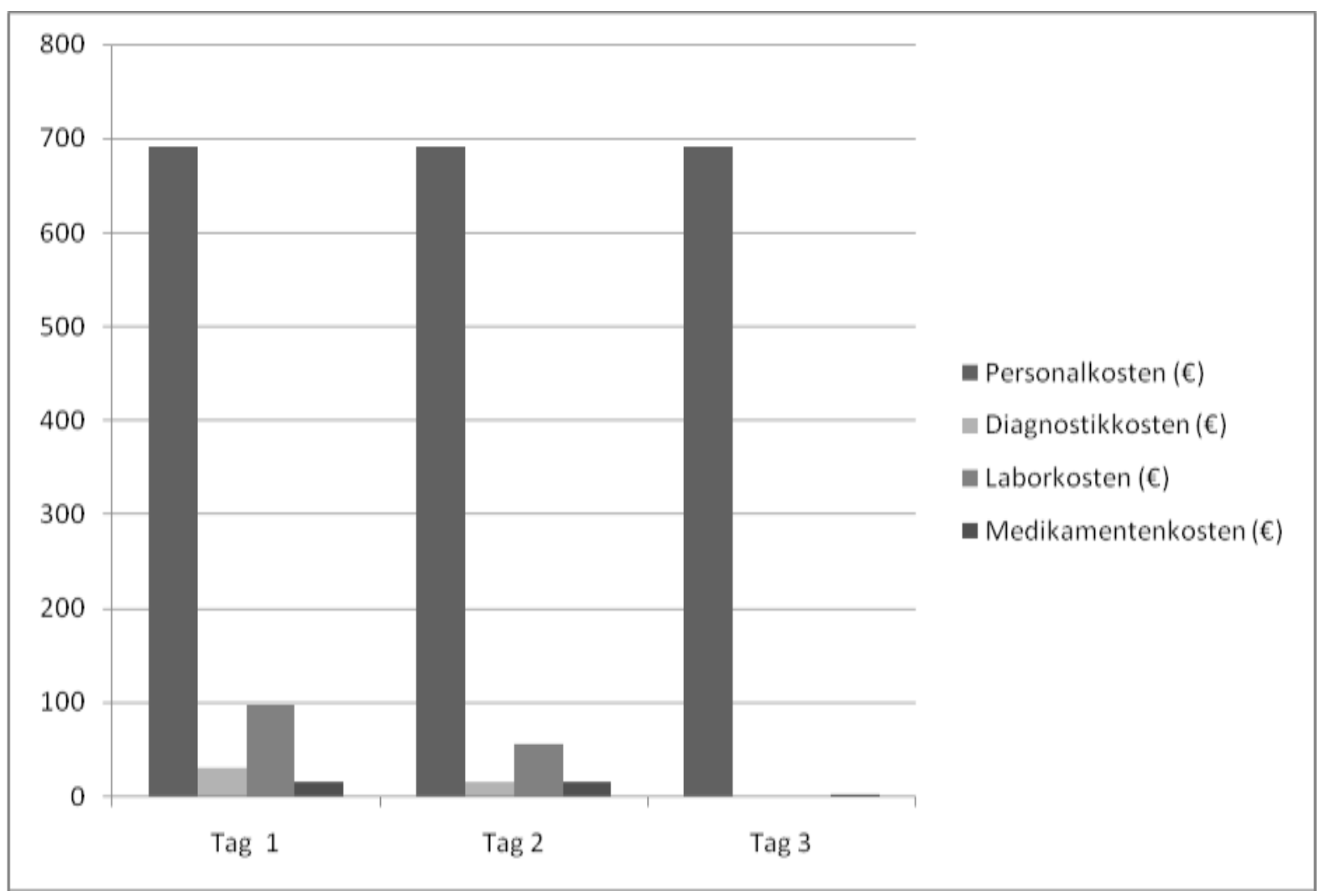

Abbildung 29: Die Verteilung der Personal -, Diagnostik -, Labor - und Medikamentenkosten des Musterpatienten B mit einer LOS von 3 Tagen, angegeben in Euro. 
Die Diagnostikkosten betrugen am ersten Tag 30,96 Euro und am zweiten Tag 15,48 Euro. Schon am dritten Tag fielen keine Diagnostikkosten mehr an. Die Laborkosten lagen an Tag eins bei 97,61 Euro und an Tag zwei bei 56,02 Euro. Am dritten Tag fielen wiederum keine Laborkosten an. Die Medikamentenkosten, welche der Musterpatient $\boldsymbol{B}$ verursachte betrugen am ersten Tag 15,12 Euro, am zweiten Tag 16,21 Euro und am dritten Tag lediglich noch 1,96 Euro.

\subsubsection{Die Verteilung der Personal -, Diagnostik -, Labor - und Medikamentenkosten des Musterpatienten $C$ mit einer LOS von 5 - 10 Tagen}

Die Verteilung der einzelnen Kostenblöcke bei einem Musterpatienten $\boldsymbol{C}$ der zweiten Patientengruppe, in welcher Patienten mit einer LOS von 5 - 10 Tagen betrachtet wurden, stellt sich wie folgt dar:

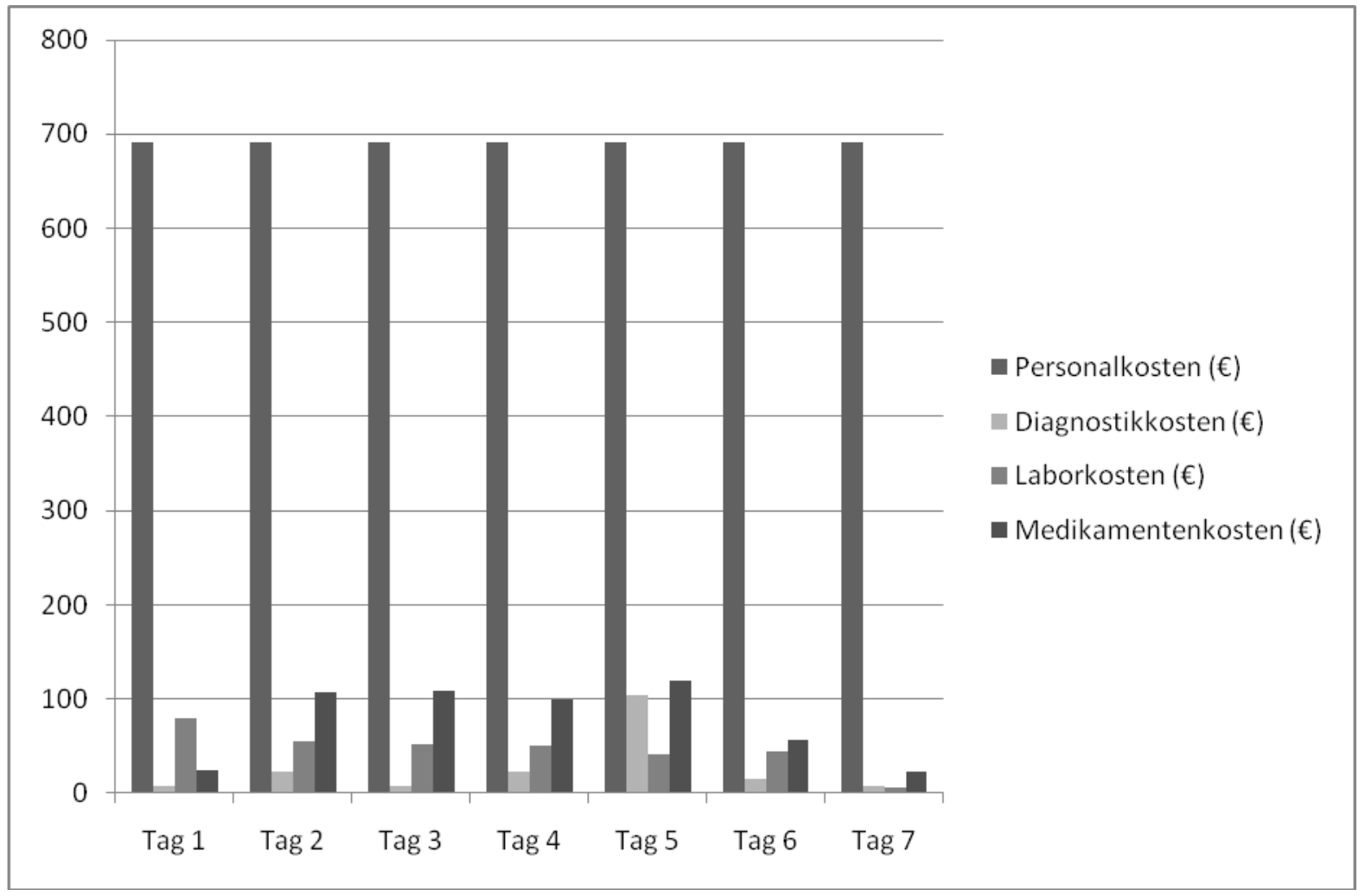

Abbildung 30: Die Verteilung der Personal -, Diagnostik -, Labor - und Medikamentenkosten des Musterpatienten $\boldsymbol{C}$ mit einer LOS von 7 Tagen, angegeben in Euro. 
Der Musterpatient $\boldsymbol{C}$ mit einer LOS von 7 Tagen verursachte täglich Personalkosten in Höhe von 691,29 Euro. Die Diagnostikkosten betrugen am ersten Tag 7,74 Euro, am zweite Tag 23,45 Euro, am dritten Tag wieder 7,74 Euro, am vierten nochmals Tag 23, 45 Euro, am fünften Tag 103,77 Euro, am sechsten Tag 15,71 und am siebten Tag wieder 7,74 Euro. Die Laborkosten waren an Tag eins 79,06 Euro, an Tag zwei 54,98 Euro, an Tag drei 52,43 Euro, an Tag vier 50,10 Euro, an Tag fünf 40,55 Euro, an Tag sechs 44,16 Euro und an Tag sieben noch lediglich 5,52 Euro. Die Medikamentenkosten, welche der Musterpatient $\boldsymbol{C}$ verursachte, betrugen 24,82 Euro am ersten Tag, 106,52 Euro am zweiten Tag, 107,99 Euro am dritten Tag, 99,31 Euro am vierten Tag, 118,72 Euro am fünften Tag, 57,02 Euro am sechsten Tag und noch 23,27 Euro am siebten Tag.

\subsubsection{Die Verteilung der Personal -, Diagnostik -, Labor - und Medikamentenkosten des Musterpatienten $D$ mit einer LOS von über 10 Tagen}

Nachfolgend wurde die Verteilung der einzelnen Kostenblöcke bei einem Musterpatienten $\boldsymbol{D}$ aus der Patientengruppe der "Langlieger", mit einer LOS von über 10 Tagen, betrachtet. Diese stellt sich wie folgt dar: 


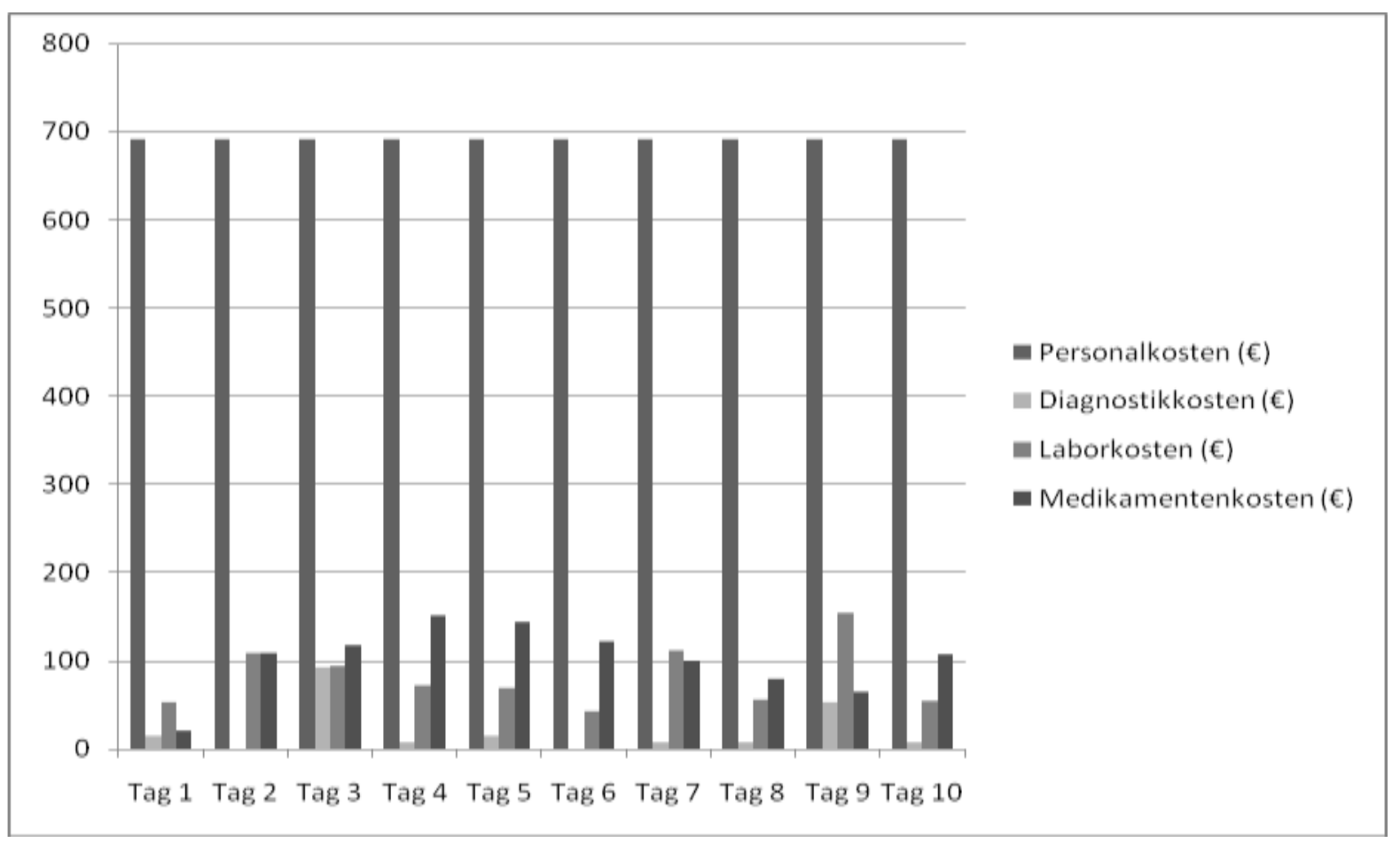

Abbildung 31a: Die Verteilung der Personal -, Diagnostik -, Labor - und Medikamentenkosten eines Musterpatienten mit einer LOS von 18 Tagen, angegeben in Euro.

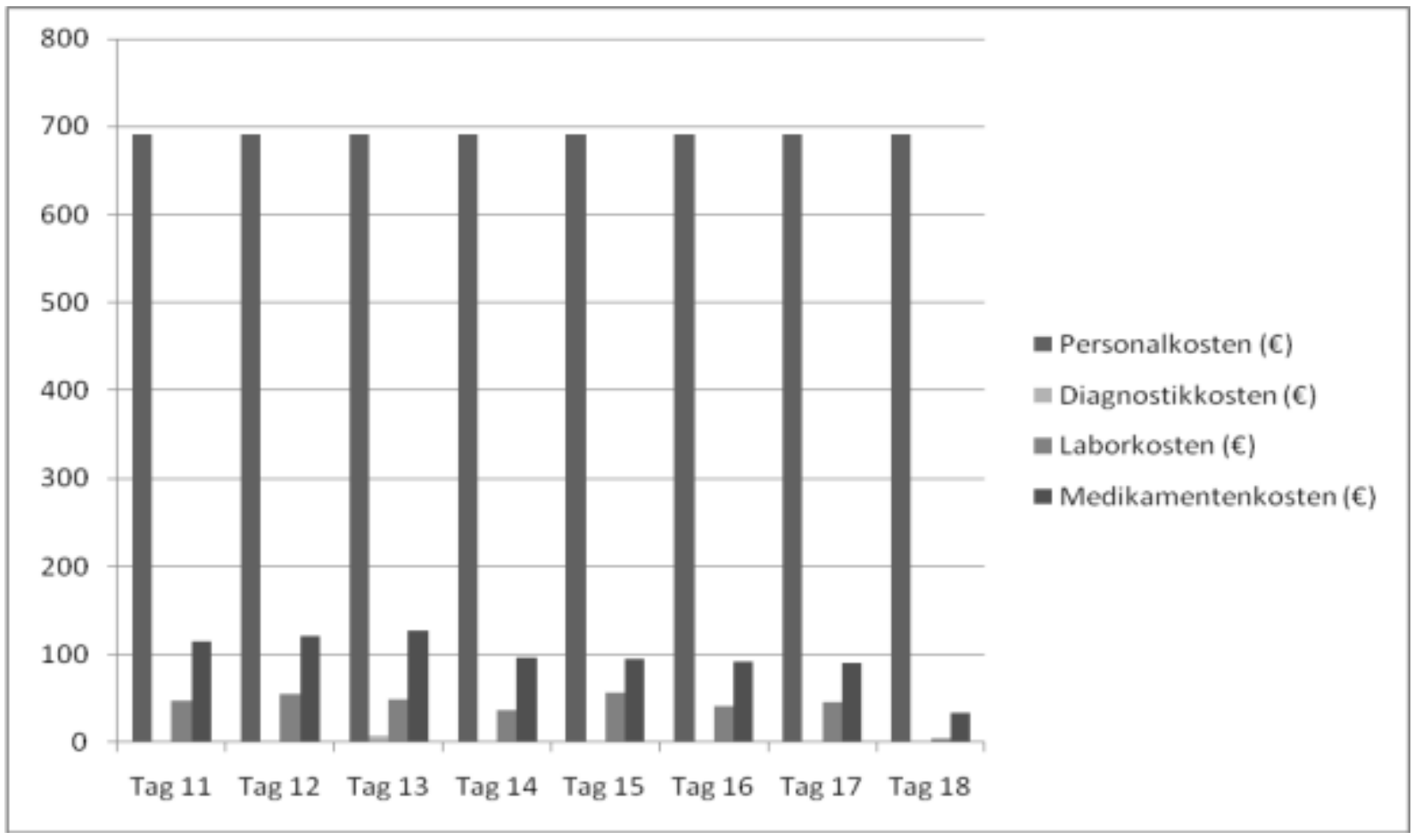

Abbildung 31b: Die Verteilung der Personal -, Diagnostik -, Labor - und Medikamentenkosten des Musterpatienten $\boldsymbol{D}$ mit einer LOS von 18 Tagen, angegeben in Euro. 
Bei diesem Musterpatienten $\boldsymbol{D}$ aus der Patientengruppe der "Langlieger" mit einer LOS von 18 Tagen betrugen die Personalkosten 691,29 Euro pro Tag. Die Diagnostik -, Labor - und Medikamentenkosten, welche für den jeweiligen Tag anfielen, sind in einer Tabelle zusammengefasst.

\begin{tabular}{|c|c|c|c|}
\hline & $\begin{array}{l}\text { Diagnostik- } \\
\text { kosten }(€)\end{array}$ & $\begin{array}{l}\text { Labor- } \\
\text { kosten }(€)\end{array}$ & $\begin{array}{l}\text { Medikamenten- } \\
\text { kosten }(€)\end{array}$ \\
\hline Tag 1 & 15,48 & 52,52 & 21,56 \\
\hline Tag 2 & 0 & 108,40 & 109,13 \\
\hline Tag 3 & 93,29 & 94,01 & 117,68 \\
\hline Tag 4 & 7,74 & 72,34 & 151,09 \\
\hline Tag 5 & 15,71 & 69,17 & 144,93 \\
\hline Tag 6 & 0 & 42,23 & 121,87 \\
\hline Tag 7 & 7,74 & 112,36 & 100,75 \\
\hline Tag 8 & 7,74 & 56,67 & 79,25 \\
\hline Tag 9 & 52,82 & 155,12 & 65,46 \\
\hline $\operatorname{Tag} 10$ & 7,74 & 55,17 & 107,01 \\
\hline Tag 11 & 0 & 47,52 & 115,37 \\
\hline Tag 12 & 0 & 55,38 & 121,13 \\
\hline
\end{tabular}




\begin{tabular}{|l|c|c|c|}
\hline Tag 13 & 7,74 & 48,58 & 126,53 \\
\hline Tag 14 & 0 & 37,13 & 96,06 \\
\hline Tag 15 & 0 & 55,81 & 94,53 \\
\hline Tag 16 & 0 & 40,73 & 91,74 \\
\hline Tag 17 & 0 & 45,41 & 90,72 \\
\hline Tag 18 & 0 & 3,61 & 32,79 \\
\hline
\end{tabular}

Tabelle 4: Die Personal -, Diagnostik -, Labor - und Medikamentenkosten des Musterpatienten $\boldsymbol{D}$ mit einer LOS von 18 Tagen, angegeben in Euro. 


\section{Diskussion}

\subsection{Die Einführung des DRG - Systems}

Mit der Umstellung der Krankenhausfinanzierung auf pauschalierte Entgelte als Leistungsvergütungssystem, welches die gesamte Behandlung eines Patienten über einen Festpreis für jede Fallgruppe vergütet, stand das deutsche Gesundheitswesen vor einem fundamentalen Paradigmenwechsel. Der Gesetzgeber hat hierzu im $\S 17 \mathrm{~b}$ des GKV - Reformgesetzes 2000 den Einsatz eines international bereits erprobten Fallpauschalensystems (Diagnosis - Related - Group (DRG) - System) vorgeschrieben. Dabei diente das australische Patientenklassifikationssystem (AR - DRG) als Grundlage für die deutsche Klassifikation von Fallpauschalen (G - DRG). Darin werden die Patienten aufgrund ihrer nach der "International Classification of Disease“ (ICD - 10) verschlüsselten Hauptdiagnose und einer nach einem Prozedurenschlüssel (OPS - 301) kodierten Hauptintervention über einen durch das Klassifikationssystem vorgegebenen Algorithmus einer spezifischen Fallgruppe zugeordnet, welche aufwandsähnliche Fälle zusammenfassen soll (Reinecke et al. 2003). Es ist also ein krankenhaus - ökonomisches und nicht ein medizinisch - wissenschaftliches Wertesystem (Burchardi 2004).

$\mathrm{Da}$ die Vergütungsform der Patientenkosten fortan pauschal und weitgehend unabhängig von etwaigen Komplikationen und damit einhergehenden Aufwandserhöhungen ausfällt, ist für viele Krankenhäuser die Deckung der Kosten problematisch.

Mit Einführung der DRGs wird auch eine angemessene Vergütung der Intensivbehandlung schwieriger, da insbesondere in den Intensivstationen der Krankenhäuser der Maximalversorgung die schwer erkrankten und aufwendig zu behandelnden Patienten innerhalb der DRG - Klassifikation versorgt werden müssen (Schmidt et al. 2004). Die Intensivmedizin gehört $\mathrm{zu}$ den teuersten Einheiten eines jeden Krankenhauses. So wurde angegeben, dass abhängig von Patientengut und Behandlungsspektrum $3-5 \%$ der stationären Patienten intensivmedizinisch behandelt wurden. Hiefür wurden, je nach Literatur, $11-25 \%$ des Krankenhausbudgets 
benötigt. So kann ein Krankenhaus, welches einen hohen prozentualen Anteil an Intensivpatienten aufweist, einen nicht unbeachtlichen wirtschaftlichen Verlust durch das DRG- Vergütungssystem erleiden (Jegers et al. 2002).

Frühere Erfahrungen in Australien und anderen Ländern zeigen sogar, dass eine angemessene Vergütung der Intensivmedizin unter DRG - Bedingungen nicht realistisch ist (Duckett 1998). In keinem der zahlreichen Länder, in denen DRGs eingeführt worden sind, werden diese zu einer vollständigen Vergütung der stationären Behandlung eingesetzt (Burchardi 2004).

Berechnungen der Abteilung für Anästhesiologie am Universitätsklinikum Göttingen ergaben, dass in den früheren Jahren nach der Einführung des neuen G - DRG - Systems durchschnittlich nur noch $47 \%$ der tatsächlich anfallenden intensivmedizinischen Kosten vergütet wurden (Neilson et al. 2004). Auf ein fast identisches Ergebnis verweist eine Studie am Universitätsklinikum München - Großhadern. Die Kosten der Behandlung operativer Intensivpatienten wurden dort durch dieses Vergütungssystem nur zu 49\% gedeckt (Billing et al. 2004). Inzwischen allerdings bildet das deutsche DRG - System durch ständige Anpassung und Weiterentwicklung die tatsächlichen Kosten der Intensivbehandlung sehr viel korrekter ab.

Die Kosten für die Intensivbehandlung zeigen eine steigende Tendenz. Die Bevölkerung wird immer älter und der medizinische Fortschritt ist inzwischen weit. Der Anreiz zur Kostenbestimmung im Gesundheitswesen wuchs mit der Erkenntnis, dass die Ressourcen begrenzt und weltweit Strategien zur Kostenbegrenzung im Gesundheitswesen entwickelt werden müssen. Zur Effizienzverbesserung in der Intensivmedizin werden zunächst dringend praktikable, akkurate und sensitive Methoden der Ressourcenmessung benötigt. Intensivpatienten sind sehr heterogen und unterscheiden sich stark in der Krankheitsschwere, Länge und Komplexität der Therapie sowie der LOS, selbst in derselben Diagnosegruppe. Zudem bezieht sich eine Intensivtherapie nicht nur auf eine spezielle Krankheit, so 
dass eine Vielfalt von Interventionen und Therapieoptionen in verschiedenen Diagnosegruppen existiert (lapichino et al. 2004).

\subsection{Die Kostenerfassung}

Sollen Scores mit finanziellen Aufwendungen verglichen werden, stellt sich die Frage der Kostenerfassung. Hierbei gibt es viele verschiedene Ansätze, Kosten zu definieren. Eine generell unter Ökonomen akzeptierte Definition von Kosten besagt: Kosten sind Mittel, die aufgewendet werden müssen, um ein spezifisches Objekt zu erhalten. Diese Aufwendungen stehen somit nicht mehr für andere Zwecke zur Verfügung.

Kosten können in verschiedene Kostenelemente (z. B. Materialkosten, Personalkosten) unterteilt werden. Dabei unterscheidet man wiederum zwischen direkten Kosten, welche komplett einem spezifischen Kostenobjekt zugeschrieben werden können, und indirekten Kosten, welche mehreren Kostenobjekten zugeordnet werden müssen.

Unternimmt man eine Kostenstudie, ist die Perspektive wichtig, aus der die Kosten betrachtet werden sollen (hier Kosten für das Krankenhaus). Wichtig ist ferner, die Art der zu ermittelnden Kosten sowie der Zeitpunkt und die Zeitspanne der Studie. Nur so können die Kosten kontrolliert und wenn nötig - überarbeitet und der Effekt von Kostenveränderungen über die Zeit beurteilt werden (Jegers et al 2002). Des Weiteren sollte die Dokumentation der Ausgaben stets standardisiert erfolgen, um Vergleiche zwischen verschiedenen Kliniken zu ermöglichen (Chaix et al. 1999).

Die "Bottom - up - Methode“ ist die Methode der Wahl zur Kostenerhebung und umfasst die individuelle Dokumentation verschiedener Kostenparameter einzelner Patienten. Diese setzen sich im Wesentlichen aus den fixen Kosten, welche sich wiederum auf die Immobilie und das Personal beziehen, und den variablen Kosten, wie beispielsweise die Kosten verbrauchter Ressourcen, zusammen (Jegers et al. 2002). 
Hierbei stellten allerdings Billing et al. bei einer Analyse am Universitätsklinikum München - Großhadern fest, dass die Kostenerfassung auf diese Weise problematisch ist. Während einige Bereiche, wie Personalkosten oder medizinischer Bedarf, durch das Klinik Controlling meist gut erfasst und abrufbar sind, werden andere Bereiche (z. B. Röntgen, Laboruntersuchungen, Physikalische Therapie) im Rahmen der hausinternen Leistungsverrechnung kaum erfasst. Aspekte wie Instandhaltung, Wirtschaftsbedarf, Reinigungskosten u. ä. werden häufig überhaupt nicht einkalkuliert (Billing et al 2004).

Eine andere Möglichkeit der Kostenerfassung besteht darin, die Gesamtkosten eines Hauses oder einer Abteilung über einen bestimmten Zeitraum hinweg zu erfassen und durch die Anzahl der in diesem Intervall behandelten Patienten zu dividieren, die sog. „Top - down - Methode“. Auf diesem Verfahren beruht zum Beispiel das von der Intensive Care National Working Group on Costs in the United Kingdom entwickelte Modell der sechs Kostenblöcke. Hierbei werden die Kosten der sechs Faktoren in sog. Kostenblöcke differenziert: Grundausstattung, Instandhaltung, Dienste nicht klinischer Mitarbeiter (wie z. B. das Krankenhausmanagement), Dienste klinisch tätiger Mitarbeiter (wie etwa Physiotherapeuten und Labormitarbeiter), Verbrauchsgüter (wie beispielsweise Pharmaka) sowie ärztliches Personal und Pflegepersonal. Auf diese Weise können die Gesamtkosten pro Patient und Tag bestimmt werden.

Abhängig von den Datenschwankungen kann diese Methode mehr oder weniger präzise sein. Eine Aussage über die Verteilung der Zusammensetzung individueller, patientenbezogener Kosten ist jedoch nicht möglich. Des Weiteren kann diese Methode nur in der Retrospektive genutzt werden (Jegers et al.2002).

Ein an die oben genannte "Top - down - Methode" angelehntes Modell zur Erfassung der durchschnittlichen Patientenkosten wählten auch Graf und seine Projektgruppe für eine Studie am Universitätsklinikum Aachen, die von November 1997 bis Februar 1998 stattfand. Um die Komplexität der 
Zusammensetzung der individuellen Patientenkosten besser erfassen zu können, teilten sie die Gesamtkosten in zwei Blöcke ein: einerseits die patienten - unspezifischen Kosten, die zunächst für jeden Patienten gleich sind und sich aus dem Mittelwert der fixen Kosten dividiert durch die Anzahl der Patiententage ergeben, und andererseits die patienten - spezifischen Kosten, welche sich auf den individuellen Ressourcenverbrauch und Interventionen beziehen und nach der "Bottom- up- Methode“ erhoben werden. Diese setzen sich im Einzelnen aus den Kosten für Pharmakotherapie, Infusionen und Transfusionen, radiologische Diagnostik, mikrobiologische, labormedizinische und pathologische Befunde sowie überdurchschnittlich kostenintensiven Therapien (wie z. B. intraaortale Ballonpumpen (IABP) oder perkutane transluminale koronare Angioplastie (PTCA) zusammen.

Die Personalkosten in patienten - spezifische und patienten - unspezifische zu unterteilen, ist schwierig, da Ärzte und Pflegepersonal inre Arbeitszeit nicht ausschließlich am Patientenbett verbringen, sondern ein Teil der Zeit z. B. für Organisation und Administration benötigt wird. So wurden in der Studie von Graf et al. die Gehälter des Personals im Dienst zu den patienten - spezifischen Kosten und die Gehälter des Personals außerhalb des Dienstes (z. B. Urlaub) zu den patienten - unspezifischen Kosten gerechnet (Graf et al. 2002).

Kostenanalysen im Gesundheitswesen sind oft auch von politischem Interesse. Daher wurden viele Studien über intensivmedizinische Kosten in den letzten Jahren publiziert.

Ein Vergleich dieser untereinander erweist sich jedoch als schwierig, da in verschiedenen Gesundheitssystemen auch verschiedene Kostenansätze angewandt werden (Chaix et al. 1999). Chaix et al. entwickelten ein Modell, welches Ärzten auf Intensivstationen helfen soll, die Kosten ihrer Patienten einzuschätzen. Dieses ist auf medizinische Kosten fokussiert, welche einfach und schnell $z u$ erfassen sind und an verschiedenen Situationen adaptiert und unabhängig von der Krankenhausstruktur sind. Es wurden nur medizinische variable Kosten betrachtet, welche alle Kosten beinhalteten, die einem bestimmten Patienten zugeordnet werden 
konnten. Personal - und andere Fixkosten wurden nicht berücksichtigt, weil diese durch die Krankenhausverwaltung leicht verfügbar sind. Für jeden Patienten wurden medizinische und demographische Daten erhoben. Somit wurden die medizinischen Kosten mit den Charakteristika eines jeden Patienten in Zusammenhang gebracht.

Chaix et al. hatten an ihr Kostenmodell den Anspruch gestellt, unabhängig von der Krankenhausstruktur zu sein, um somit einen Vergleich verschiedener Krankenhäuser zu ermöglichen. Da ihrer Meinung nach die totalen Kosten einfach durch das Summieren der Personal - und Fixkosten zu ermitteln sind, wurde in ihrem Kostenmodell der Betrachtungsschwerpunkt auf die medizinischen (variablen) Kosten gerichtet. Im Gegensatz dazu, wurden jedoch in der vorliegenden Studie die Gesamtpatientenkosten betrachtet. Diese totalen Kosten entstehen durch das Addieren von medizinischen Kosten, Personalkosten und Fixkosten, wovon die medizinischen Kosten am schwierigsten zu erfassen sind.

Das Kostenmodell von Chaix et al. ist unabhängig von der Krankenhaustruktur. Daher kann es in jeder ICU angewendet werden und später die von der eigenen Krankenhausstruktur abhängigen Personalund Fixkosten addiert werden. Dieses standardisierte Kostenmodell erlaubt somit einen Vergleich verschiedener Intensivstationen mit ihren Kosten. Des Weiteren kann es sensitiver als eine DRG - basierte Vergütung sein, welche eine Trennung von intensivmedizinischen Kosten und anderen Krankenhauskosten nicht erlaubt.

Einen ähnlichen Ansatz der Kostenanalyse für Intensivpatienten benutzen auch Slatyer et al. in ihrer Studie 1986. Die Kosten dieser Studie waren im Vergleich zu der Studie von Chaix et al. dreimal niedriger. Dies spiegelt, auch unter Berücksichtigung der Inflationsrate, den generellen Zuwachs der Therapie - und Diagnostikkosten in den letzten Jahrzehnten wider.

Auch in der vorliegenden Studie wurden für jeden Patienten medizinische und demographische Daten erfasst: Alter, LOS, Geschlecht, SAPS II, 
TISS - 28, Diagnose. Die direkten, patientenbezogenen (variablen) Kosten wurden nach der Bottom - up - Methode ermittelt. Diese so erfassten variablen Kosten setzen sich aus den Kosten für Medikation, Blutprodukte, Ernährung, Monitoring, Labor und Mikrobiologie, Diagnostik, Beatmung oder Dialysebehandlung des individuellen Patienten zusammen. Sie sind also direkte Kosten, die dem Krankenhaus tatsächlich entstanden sind. Die fixen Kosten dagegen wurden nach der Top - down - Methode erfasst. Sie wurden aus den Daten der Krankenhausveraltung ermittelt. Diese fixen Kosten (jeweils pro Tag) sind einerseits die Personalkosten und andererseits Overheadkosten, welche u. a. Strom, Heizung, Verwaltungskosten beinhalten. Auch bei den Personalkosten handelt es sich um direkte Ausgaben des Krankenhauses. Die Overheadkosten sind dagegen Erstattungsanteile, die mit den Kassen ausgehandelt wurden. Um die totalen Kosten zu erhalten, werden variable Kosten und Fixkosten pro Tag und Patient addiert.

\subsection{Kostenvergleiche}

Eine standardisierte Methodik zur Messung der entstehenden intensivmedizinischen Kosten hat sich bisher nicht etabliert (Gyldmark 1995). Insofern sind weitere Vergleiche zwischen den Daten der vorliegenden Arbeit und jenen anderer Untersuchungen bezüglich der intensivmedizinischen LOS und Gesamtkosten schwierig. Zudem unterscheiden sich die Studien hinsichtlich der Ein - und Ausschluss kriterien oft erheblich. Dies erschwert zusätzlich einen Vergleich der Ergebnisse verschiedener Untersuchungen.

In letzter Zeit wurden bundesweit mehrere Studien durchgeführt mit dem Ziel der genauen Kostenerfassung, Recherchen hinsichtlich der Möglichkeiten der Kostensenkung und im Endeffekt auch der Erarbeitung einer objektiven Grundlage für die Vergütung intensivmedizinischer Leistungen. Zu diesem Studienkomplex zählt auch die gemeinsam von der DIVI und dem VUD durchgeführte „VUD - Kostenstudie“, eine Multicenterstudie an 9 Universitätskliniken mit insgesamt 17 
Intensivstationen, in die auch die Ergebnisse der vorliegenden Arbeit eingeflossen sind. Im Rahmen dieser Kostenstudie sollte die Möglichkeit der Vergütung intensivmedizinischer Kosten anhand der bestehenden Scores SAPS II und TISS - 28 geprüft werden (Moerer et al. 2007).

In das Gesamtkollektiv der vorliegenden Untersuchung von 100 Personen flossen Daten von Patienten einer internistischen Intensivstation ein, die das 18. Lebensjahr vollendet hatten und mindestens 24 Stunden auf der Intensivstation behandelt wurden. Die Daten chirurgischer Intensivpatienten wurden in einer anderen Studie gesondert betrachtet. Das Durchschnittsalter der Patienten der vorliegenden Studie von 61,5 Jahren liegt bemerkenswerterweise zwischen dem Wert von 59,0 Jahren in einer vergleichbaren Erhebung am Universitätsklinikum Brüssel (Ferreira et al. 2001) und dem Wert von 65,5 Jahren in einer in Deutschland auf Basis der Matrix des Institutes für das Entgeltsystem im Krankenhaus (InEK) durchgeführten Kostenanalyse intensivmedizinischer Behandlung (Martin et al. 2008). In Ferreiras Untersuchung und auch in jener von Martin galten die gleichen Ein - und Ausschlusskriterien wie in der vorliegenden Studie. Jedoch wurden beide in anästhesiologischen Intensivstationen durchgeführt, welche vorwiegend chirurgische Patienten behandeln. Die durchschnittliche intensivmedizinische Liegedauer (LOS) war mit 8,1 Tagen in Martins Studie höher als beim Patientenkollektiv dieser Arbeit mit 5,6 Tagen.

Die VUD - Studie beruht auf der mühsamen Bottom - up - Methode, die einzig zuverlässige Kostenanalyse, welche die Kostenvariabilität eines einzelnen Patienten berücksichtigt. (Moerer et al. 2002). Analog wurde auch in anderen Studien (Moerer und Burchardi 2001, Moerer et al. 2002, Staudacher et al. 2003) verfahren.

Die durchschnittlichen Gesamtkosten pro Fall betragen in Staudachers Studie 12.666 DM (Kostenzeitpunkt 2000) (Staudacher et al. 2003). Dieser Wert liegt wieder sehr nahe an den in der vorliegenden Studie berechneten Gesamtkosten pro Fall von 6.954 Euro. Allerdings wurden 
auch beide Studien am Universitätsklinikum Göttingen erhoben, die Staudacher - Studie in der operativen Intensivmedizin.

Es erscheint aber bemerkenswert, dass die täglichen intensivmedizinischen Patientenkosten in dieser Studie mit durchschnittlich 1.196 Euro sehr nahe an den Werten anderer Publikationen deutscher Intensivstationen liegen. Diese werden mit 1.336 Euro pro Tag (Kostenzeitpunkt 1998) (Graf et al. 2002), 1.318 Euro (Kostenzeitpunkt 1999, Sepsis - Patienten!) (Moerer et al. 2002), 1.265 Euro pro Tag (Kostenzeitpunkt 2006) (Martin et al. 2008) und 1.050 Euro pro Tag (Kostenzeitpunkt 2000) (Billing et al. 2004) angegeben. Die täglichen Kosten einer internistischen Intensivstation mit vorwiegend kardiologischen Patienten belaufen sich in der Studie von Klepzig et al. auf 1.444 Euro (Kostenzeitpunkt 1997) (Klepzig et al. 1998). Auch im internationalen Vergleich sind die täglichen Behandlungskosten pro Patient in der Intensivmedizin ähnlich: in Großbritannien zwischen 1.396 Euro (Kostenzeitpunkt 1996) und 1.647 Euro (Kostenzeitpunkt 1994) (Edbrooke et al. 1999 und Dickie et al. 1998) und in Kanada 1.007 Euro (Kostenzeitpunkt 1992) (Noseworthy et al. 1996).

\subsection{Der Einfluss der Patientenliegedauer}

Frühere internationale Studien haben gezeigt, dass Patienten mit einer langen ICU - LOS ein hohes Risiko für ein schlechtes Outcome haben und hohe Kosten produzieren.

So waren $9 \%$ aller Patienten der Studie von Heyland et al. länger als 14 Tage auf der Intensivstation. Diese "Langlieger" (LOS über 14 Tage) hatten eine durchschnittliche LOS von 24,5 Tagen. Sie unterschieden sich in ihren Diagnosen (vorwiegend kardiologische, respiratorische und Traumapatienten) von den Patienten mit einer kürzeren LOS. Des Weiteren hatten sie verständlicherweise einen höheren Krankheitsschwerescore. Die ICU - Gesamtkosten waren bei den "Langliegern“ erheblich höher (25.200 Euro) im Vergleich zu der Patientengruppe mit einer relativ kurzen LOS (2.860 Euro). In der Studie von Heyland et al. 
wurden nicht die posthospitalen Folgekosten, wie beispielsweise die Rehabilitation bei „Langliegern“, berücksichtigt. Dann würden die Kosten für das Gesundheitssystem für "Langlieger" noch ausgiebiger ansteigen (Heyland et al. 1998).

Auch Staudacher et al. untersuchten in ihrer Kostenstudie am Universitätsklinikum Göttingen potentielle Kostenindikatoren intensivmedizinischer Kosten (Staudacher et al. 2003). Es zeigte sich ein signifikanter Unterschied der mittleren Kosten bei Patienten mit einem SAPS $\|<30$, SAPS $\| 30-60$ und SAPS $\|>60$. Des Weiteren findet sich eine lineare Beziehung zwischen LOS und Kosten sowie zwischen LMV (LMV = Length of Mechanical Ventilation) und Kosten, wobei die Korrelation zwischen LOS und Kosten $(r=0,9)$ höher war als die Korrelation zwischen LMV und den Kosten $(r=0,68)$.

In dieser Studie konnten somit zwei wichtige Kostenindikatoren ausgemacht werden: LOS und LMV. Die LOS ist jedoch der präzisere Indikator, da zum einen zwischen ihr und den Kosten eine höhere Korrelation besteht und zum anderen die LMV nur bei beatmeten Patienten herangezogen werden kann (Staudacher et al. 2003).

Bereits 1977 zeigte Cullen, dass der Kostenaufwand für den Einzelfall neben der Erkrankungsschwere und dem dadurch bedingten diagnostischen und therapeutischen Aufwand, vor allem aber durch die Verweildauer (LOS), bestimmt wird. Das Ziel einer Pilotstudie von Neilson et al. in der anästhesiologischen Intensivstation der Universitätsklinik Göttingen war es, die Aussagekraft der LOS als eine Variable für die direkten totalen Kosten pro Fall in der Intensivmedizin zu prüfen und somit ein realistisches Vergütungssystem für intensivmedizinische Kosten zu entwickeln (Neilson et al. 2004). Die Analyse ihrer Daten ergab, dass $83 \%$ der Patienten eine LOS von bis zu 7 Tagen aufwiesen und dabei lediglich $44 \%$ der Kosten verursachten, während bei 15\% der Patienten mit einer LOS zwischen 7 und 20 Tagen $33 \%$ der Kosten entstanden. Die Langzeitintensivpatienten mit einer LOS von mehr als 20 Tagen, welche nur $2 \%$ aller Intensivpatienten 
repräsentierten, verursachten beträchtliche $23 \%$ der Intensivstationskosten. Somit verbrauchten also $17 \%$ aller Intensivpatienten bereits $56 \%$ der Kosten.

Bei der in dieser Arbeit vorgenommenen Aufteilung der Gesamtkosten in die einzelnen Kostenblöcke der täglichen Personal -, Diagnostik -, Labor und Medikamentenkosten anhand der Musterpatienten fällt auf, dass die Personalkosten an jedem Behandlungstag bei allen betrachteten Patienten den Hauptteil der Kosten ausmachen. Sie betragen konstant 691,29 Euro pro Tag. Die täglichen Diagnostik -, Labor - und Medikamentenkosten fallen gerade am Anfang des Behandlungszeitraumes an und sind dann gegen Ende der Behandlung verschwindend gering.

Die in der vorliegenden Arbeit nachgewiesenen unterschiedlichen Korrelationen zwischen dem Gesamt - SAPS II bzw. Gesamt - TISS - 28 und den Gesamtkosten in den drei verschiedenen LOS - Patientengruppen erklären sich somit. In der ersten Patientengruppe, in welcher Patienten mit einer LOS unter 5 Tagen betrachtet wurden, fallen die Diagnostik -, Labor - und Medikamentenkosten (am Anfang der Behandlung) gegenüber den immensen Personalkosten noch ins Gewicht. In der Patientengruppe der "Langlieger" nehmen die Personalkosten einen prozentual immer größeren Anteil an den Gesamtkosten ein. Mit der steigenden Anzahl an Behandlungstagen (LOS) entsteht durch den gewichtigeren Einfluss der Personalkosten folglich eine immer höhere Korrelation zwischen dem Gesamt - SAPS II bzw. dem Gesamt - TISS - 28 und den Gesamtkosten. Man kann sogar sagen, dass mit steigender Anzahl an Behandlungstagen (LOS) die Korrelation zwischen den Gesamtscorewerten und den Gesamtkosten der Korrelation zwischen der LOS und den Gesamtkosten annähernd entspricht.

Denkbar wäre also, Vergütungskonzepte zu entwickeln, in denen die Kostendeckung bis zu einer bestimmten intensivstationären Verweildauer (Grenzverweildauer) über das G - DRG - System geregelt ist und bei 
Überschreiten der Grenzverweildauer durch Zusatzentgelte fortgeführt wird. Diese könnten an Scores geknüpft werden, da sich unvorhergesehene Komplikationen, welche die kalkulierte Verweildauer erhöhen, in den Scores durch erhöhte Krankheitsschwere und Pflegeaufwendungen niederschlagen würden.

Neilson et al. zeigten, dass in ihrer Studie ab einer LOS $>7$ Tage eine DRG - basierte Vergütung unter den derzeitigen Bedingungen nicht mehr ausreichend war und ab dem 7. Tag eine zusätzliche Vergütung erfolgen sollte. Patienten mit einer LOS von > 20 Tagen sollten sogar eine „outlier DRG - Klassifikation" erhalten (Neilson et al. 2004). Auch von Billing und Mitarbeitern wird die Schaffung einer oberen Grenzverweildauer für die Intensivbehandlung mit anschließender Vergütung durch zusätzliche Tagesentgelte vorgeschlagen (Billing et al. 2004). Dieses war jedoch bei der Einführung des DRG - Systems seinerzeit politisch nicht gewünscht.

Wie eine finnische Studie von Parviainen et al. zeigt, konnte durch das Einführen von Guidelines für Sedation eine durchschnittlich kürzere Beatmungszeit und LOS und somit auch eine Kostenreduktion erzielt werden (Parviainen et al. 2004). Dies zeigte, dass eine Kostenreduktion auch trotz oder sogar durch eine Qualitätsverbesserung erfolgen kann. Wie also in mehreren Studien gezeigt wurde, sind die intensivmedizinischen Kosten abhängig von der Krankheitsschwere und der LOS. Komplikationen, wie beispielsweise eine Beatmung oder invasives Monitoring führen zu einer längeren LOS und somit auch zu höheren intensivmedizinischen Kosten. 


\subsection{Die Personalkosten}

In der Studie von Edbrooke und seinen Mitarbeitern haben sich die Personalkosten (55\% der Gesamtkosten) als ein Hauptkostenfaktor herausgestellt (Edbrooke et al. 1999). Auch in den Untersuchungen von Martin et al., Moerer et al. und Klepzig et al. liegen die Personalkosten zwischen 50\% und 55\% der Gesamtkosten recht nahe beieinander (Martin et al. 2008; Moerer et al. 2007; Klepzig et al. 1998). Sie betragen in der Studie von Noseworthy et al. sogar 63,8\% der Gesamtkosten, davon sind 41,2\% Kosten für Krankenpflege, 9,6\% Kosten für ärztliches Personal und $13 \%$ für andere Berufsgruppen (Noseworthy et al. 1996).

Die Deutsche Krankenhausgesellschaft teilte für das Jahr 2005 einen Personalkostenanteil für Arzt, Pflege und Funktionsdienst im Durchschnitt über alle deutschen Krankenhäuser von 53,1\% mit. Dies ist bemerkenswerterweise sehr nahe dem in der vorliegenden Arbeit ermittelten Personalkostenanteil von $57,8 \%$.

Es ist charakteristisch, dass in allen hochzivilisierten Ländern mit anspruchsvoller Intensivmedizin für das Personal zwischen 45\% und 65\% des Budgets der Station ausgegeben wird (Burchardi 2004). Dies sind Fixkosten, d.h., sie fallen mit jedem Behandlungstag an, unabhängig von der tatsächlichen Inanspruchnahme. Durch Verkürzung der LOS auf der Intensivstation ließen sich also entscheidende Kosteneinsparungen erreichen. Ein Abbau von Personal über einen Grenzwert hinaus kann sich jedoch auf Intensivstationen kontraproduktiv auswirken. Auf Intensivstationen müssen schwerstkranke Patienten rund um die Uhr versorgt werden. Bei übergroßer Arbeitsbelastung konnte ein Anstieg der Mortalität nachgewiesen werden (Tarnow - Mordi et al. 2000).

Dabei zeigt die ärztliche Präsenz in einer Studie von Stiletto et al. auf deutschen Intensivstationen ohnehin schon deutliche Defizite. So gab es in $44 \%$ der befragten Intensivstationen nachts nicht einmal einen Assistenzarzt. Zudem war die LOS auf der Intensivstation bzw. im 
Krankenhaus bei Patienten, welche durch intensivmedizinisch geschulte Ärzte umfassend betreut wurden, deutlich kürzer (Stiletto et al. 2000).

Unter diesen Gesichtspunkten erscheint die unreflektierte Senkung der Personalausstattung einer Intensivstation als ein wenig geeignetes Instrument zur nachhaltigen Sanierung der Kostenstruktur.

Die Forderung nach einer IMC („Intermediate Care“ - Stationen) als „puffernde" Zwischenstation wird somit zunehmend dringend, wenn die LOS auf Intensivstationen verkürzt werden soll. Auch Institutionen der Frührehabilitation können entscheidend zur Verkürzung der intensivmedizinischen Verweildauer beitragen (Burchardi 2004).

\subsection{Die Diagnose - bezogenen Kosten}

In mehreren Kostenstudien zeigte sich, dass die durchschnittlichen direkten, patientenbezogenen (variablen) Kosten (ohne Personalkostenanteil) stark abhängig von der Diagnose sind. In der Studie von lapichino et al. waren die Behandlungs - Fallkosten in den verschiedenen Diagnosegruppen in absteigender Höhe wie folgt verteilt: traumatologische Patienten 4.422 Euro, ARDS - Patienten 3.645 Euro, ungeplante abdominalchirurgische Patienten 3.082 Euro, Hirntraumapatienten 2.934 Euro, Hirnblutung 2.549 Euro, Exacerbation bei COPD Patienten 1.884 Euro, kardiologische Patienten 1.323 Euro, Strokepatienten 969 Euro, geplant abdominalchirurgische Patienten 632 Euro (lapichino et al. 2004).

In dieser Kostenstudie von lapichino et al. wie auch bei Noseworthy et al. fanden sich beachtliche Unterschiede in den Kosten zwischen geplanten und notfallmäßigen chirurgischen Patienten (Noseworthy et al. 1996).

In einer Studie von Moerer et al. an den Universitätskliniken Göttingen, Halle und Jena, welche bei Sepsispatienten die einzelnen Kostenblöcke betrachtete, setzten sich die totalen intensivmedizinischen Kosten aus Medikamentenkosten (9.304 Euro), Laborkosten (2.337 Euro), Mikrobiologiekosten (882 Euro), Verbrauchsgegenstände (753 Euro), 
Overheadkosten (1.518 Euro) und Personalkosten (8.503 Euro) pro Patient zusammen. Den größten Teil der Gesamtkosten macht somit die Medikation (40\%), gefolgt von den Personalkosten (36\%) aus (Moerer et al. 2002).

Die Kosten der einzelnen Kostenblöcke in Moerers Studie sind jedoch deutlich höher, als die in der vorliegenden Untersuchung, denn Sepsispatienten haben einen hohen Krankheitsschweregrad mit komplexem Multiorganversagen und eine lange LOS, was sich in den Kosten widerspiegelt. Dennoch konnten auch in unserer Arbeit bei niedrigerem Gesamtkostenniveau die gleichen größten Kostenblöcke Medikation (29\%) und Personalkosten (57,8\%) aufgezeigt werden.

Auch in einer Multicenterstudie, welche im Jahr 2003 an 51 deutschen Intensivstationen von Moerer und seinen Mitarbeitern durchgeführt wurde, variierten die täglichen Kosten in den einzelnen Patientengruppen stark (Moerer et al., 2007). So waren auch hier notfallmäßig chirurgische Patienten teurer als geplant chirurgische oder nichtchirurgische Patienten. Zudem waren beatmete Patienten und Patienten mit einer Sepsis teurer. $10 \%$ der Patienten verursachten die höchsten täglichen Kosten, diese Patienten hatten ein Casemix aus notfallmäßig chirurgischen, beatmeten und septischen Patienten.

Auch konnten Moerer et al. in ihrer Studie eine klare Gruppentrennung der Kosten bei Patienten mit einem SAPS II $<47$ (742 Euro) versus SAPS II $\geq 47$ (984 Euro) finden (Moerer et al. 2007).

In der vorliegenden Arbeit, mit zu 70\% kardiologischen Patienten, fanden sich durchschnittliche direkte, patientenbezogene Tageskosten von 1.196 Euro. Dieser Wert liegt nahe an dem in der Studie von lapichino et al. ermittelten Wert von 1.323 Euro (Kostenzeitpunkt 1999) für kardiologische Patienten (lapichino et al. 2004).

Bei der Entwicklung des SAPS waren primär kardiologische Patienten ausgeschlossen. Grund hierfür war vor allem eine nicht genügend große Zahl dieser Patienten bei der ursprünglichen Validierung dieses Scores. 
Außerdem wurde erwartet, dass Patienten mit einem akuten Myokardinfarkt einen hohen Scorewert erreichen, hieraus jedoch im Vergleich zu anderen Patientengruppen keine entsprechend hohe Letalität resultiert.

Spätere Arbeiten konnten inzwischen jedoch zeigen, dass der SAPS auch bei kardiologischen Patienten zu validen Ergebnissen bezüglich Mortalität und Krankheitsschwere führt.

In spanischen Krankenhäusern sind die instabile Angina pectoris und der Verdacht auf einen akuten Myokardinfarkt (AMI) immerhin bei 35\% der Patienten der Grund der Zuweisung auf eine Intensivstation, 75\% davon erleiden tatsächlich einen AMI.

Daher verglichen Reina et al. in einer 1992 an 70 spanischen Krankenhäusern durchgeführten Studie den SAPS II als Prognosesystem für Mortalität bei Intensivpatienten mit AMI. Sie fanden heraus, dass der Score die Mortalität und die Krankheitsschwere auch für Patienten mit AMI akkurat beurteilt, obwohl er ursprünglich kardiologische und kardiochirurgische Patienten ausschließt (Reina et al. 1996).

Auch in einer Studie von Schuster et al. wurden in einem deutschen Lehrkrankenhaus kardiologische und kardiochirurgische Patienten hinsichtlich der Anwendung des SAPS II an diesem Patientenkollektiv untersucht. Auch sie schlussfolgerten, dass in ihrer Studie der SAPS II genauso valide bezüglich der Mortalitätswahrscheinlichkeit und Krankheitsschwere für kardiologische und kardiochirurgische Patienten ist (Schuster et al. 1997).

Reinecke et al. untersuchten in ihrer Studie an drei aus medizinischer und ökonomischer Sicht für die Kardiologie besonders bedeutsamer Fallgruppen die Vergütung anhand der für diese Fallgruppen kalkulierten G - DRG (Reinecke et al. 2003). In einer der Fallgruppen kam es zu Kosteninhomogenitäten durch besonders kranke, multimorbide Patienten. Allerdings führte dies nicht zu einer Steigerung des ökonomischen Schweregrades mit Einfluss auf die Leistungsvergütung. Zur Lösung 
dieser Problematik wäre ein Schweregradsplitting denkbar. Eine andere Lösung wäre, die Erhöhung der Vergütung bei Überschreitung einer oberen Grenzverweildauer (Reinecke et al. 2003), wie sie auch von Neilson und seinen Mitarbeitern (Neilson et al. 2004), sowie von Billing und seinen Mitarbeitern (Billing et al. 2004) vorgeschlagen wurde.

In einer anderen Fallgruppe waren der Verbrauch von Material und Medikamenten, wie z. B. Gp Ilb/ IIla - Inhibitoren, nicht ausreichend berücksichtigt. Ferner waren seinerzeit in den $G$ - DRG aufwändige Leistungen, aber insbesondere auch innovative neue Verfahren, nur ungenügend berücksichtigt. Zudem bestanden hier unzureichende Verschlüsselungscodes (Reinecke et al. 2003).

In der Studie von Reinecke et al. wurden deutliche Diskrepanzen zwischen entstehenden Kosten und zu erwartenden Erlösen bei der Behandlung aufwändiger Patienten festgestellt. Kliniken, welche einen höheren Anteil dieser Patienten behandeln, sind daher angesichts des Ausmaßes des Defizits von einer deutlichen Unterfinanzierung bedroht. Somit bestand seinerzeit für die kardiologischen G - DRGs sowohl bei den Diagnose - und Prozeduren - Klassifikationssystem sowie der medizinisch und ökonomisch sinnvollen Definition der G - DRG - Fallgruppen noch erheblicher Nachbesserungsbedarf (Reinecke et al. 2003).

Die Definition des „akuten“ Myokardinfarkts (AMI), welche die Patienten in diese G - DRG führt, ist unter Berücksichtigung der aktuellen Kodierrichtlinien erfüllt, wenn der AMI innerhalb der letzten 28 Tage aufgetreten war. Diese Definition benachteiligt Kliniken mit einer 24h - PTCA - Bereitschaft, weil sie ein besonders kritisch krankes und somit auch teureres Patientenkollektiv (z. B. im kardiogenen Schock) zeitnah dem Ereignis behandeln. Hieran wird besonders das Problem der klinischen Schwerpunktbildung bei mangelnder Berücksichtigung dieser medizinischen Leistungen im G - DRG - System deutlich (van de Werf et al. 2003).

In den letzten Jahren ist jedoch das deutsche DRG - System weiterentwickelt worden. Federführend in dieser Entwicklung ist das 
InEK (Institut für das Entgeltsystem im Krankenhaus $\mathrm{GmbH}$ ). Durch Einführung der neuen Komplexziffern (z. B. 8-980 Intensivmedizinische Komplexbehandlung) hat sich jetzt die Vergütung der intensivmedizinischen Leistungen wesentlich verbessert und bietet unter günstigen Umständen Kostendeckung.

\subsection{Verfahrenskritik}

Die als vordergründig befriedigend erscheinenden Ergebnisse der vorliegenden Arbeit müssen jedoch teilweise durch die Einschränkungen der Methodik relativiert werden. So wirkte sich auf die Datenerhebung limitierend aus, dass aufgrund begrenzter Dokumentationskapazitäten nicht immer alle Patienten in die Studie aufgenommen werden konnten. $\mathrm{Da}$ in diesen Zeiträumen die Aufnahme neuer Patienten in die Studie randomisiert erfolgte, wurde die Repräsentativität des Patientenkollektives dennoch aufrechterhalten.

Wie bereits hinsichtlich der allgemeinen Problematik der Scoreerhebung erwähnt, standen in seltenen Fällen einige zur Scoreerhebung notwendigen Parameter nicht zur Verfügung. Hierunter fallen vor allem einige wenige Laborparameter, welche nicht jeden Tag bestimmt wurden. In den Scoreberechnungen galt dann erneut der am Vortag erhobene Laborwert. Diese Vorgehensweise erscheint jedoch vertretbar, da Laborwerte oft nicht bestimmt werden, wenn nicht von kontrollpflichtigen pathologischen Schwankungen jener Werte auszugehen ist.

Des Weiteren wurden in der vorliegenden Studie ausschließlich Patienten betrachtet, welche mehr als 24 Stunden auf der Intensivstation behandelt wurden. So kann es sein, dass extrem schwer kranke Patienten (und somit auch vermutlich teurere), welche in den ersten Stunden nach Einweisung verstarben nicht in die Studie eingingen.

$\mathrm{Da}$ in der vorliegenden Studie allein eine internistische Intensivstation eines Krankenhauses der Maximalversorgung untersucht wurde, können die Ergebnisse nicht ohne weiteres auf andere Intensivstationen 
übertragen werden. So weisen Moerer et al. auf mögliche Kostenunterschiede zwischen den einzelnen Versorgungsstufen hin (Moerer et al. 2007). Auch muss die Übertragung der Schlussfolgerungen dieser Studie auf andere Studien kritisch interpretiert werden, obwohl wir glauben, dass die Kosten für Personal und Ressourcenverbrauch auf anderen Intensivstationen ähnlich sein werden.

\subsection{Die Entwicklung eines kostengerechten Aufwandmarkers}

In der vorliegenden Arbeit wurde von der Hypothese ausgegangen, dass die Kostenerstattung an einen Krankheits - und Aufwandsmarker SAPS II und TISS - 28 gekoppelt werden könnte, um eine objektive Bemessungsgrundlage für die Vergütung intensivmedizinischer Leistungen zu entwickeln. In der Vergangenheit hat es zwar häufig diverse Evaluierungen hinsichtlich ihrer medizinischen, jedoch kaum Untersuchungen zu deren ökonomischer Aussagekraft gegeben. Jedoch ist es aus medizinischer, ethischer und finanzieller Sicht erforderlich, eine frühe Abschätzung bezüglich des Erfolges der therapeutischen Maßnahmen zu erlangen.

Scoresysteme stellen, gerade in Zeiten des Kostendrucks in der Intensivmedizin, eine Möglichkeit dar, das Patientenkollektiv zu beschreiben, aus der Behandlung entstehende Kosten transparent zu machen, sowie die Effizienz der Therapie darzustellen und vergleichend zu evaluieren. Es ist daher sinnvoll, dass in der vorliegenden Studie auch die prinzipielle Eignung der Scores SAPS II und TISS - 28 in dieser Hinsicht einer Überprüfung unterzogen wurde.

Es ist in zunehmendem Maße wichtig, die Abläufe auf Intensivstationen zu beurteilen, um effektive und schließlich effiziente Kriterien für den Patienten zu schaffen. Der TISS - 76, welcher 1974 von Cullen et al. entwickelt wurde, war hierbei ein großer Durchbruch. Obwohl er anfangs etabliert wurde, um das Ausmaß der Krankheit zu erfassen, bekam er später größere Bedeutung, um Ressourcen zu messen (Moreno und Morais 1997). Heute dient der TISS schwerpunktmäßig dazu, 
die Höhe der Punktzahl in einen Zusammenhang mit dem Therapieaufwand, insbesondere dem zeitlichen Pflegeaufwand und damit indirekt mit dem Personalbedarf von Intensivstationen zu bringen.

Die originale Publikation des TISS von Cullen et al. 1974 beinhaltet eine kleine Studie, welche schon eine Korrelation zwischen dem TISS und den Kosten zeigt (Cullen et al. 1974). Des Weiteren findet sich auch in einer detailierteren und größeren Studie von Slatyer und seinen Mitarbeitern eine lineare Beziehung zwischen dem TISS und den ICU - Kosten (Slatyer at al. 1986).

In London ermittelte die Arbeitsgruppe um Dickie einen Wert von umgerechnet 39,90 Euro pro TISS - Punkt (inklusive Personalkosten und Overhead, Kostenzeitpunkt 1994). Die Korrelation zwischen dem TISS und den totalen Kosten ergab einen Korrelationskoeffizienten von 0,93 (Dickie et al. 1998).

Bei der engen Korrelation zwischen TISS und intensivmedizinischen Behandlungskosten, liegt es nahe, die Gesamtpunktzahl als Summe der täglichen TISS - Werte über die gesamte intensivmedizinische Behandlungsdauer mit den Gesamtkosten zu vergleichen.

An den Universitätskliniken in Köln und Aachen wurden die intensivmedizinischen Kosten (inklusive Personalkosten und Overhead) analysiert und mit der Krankheitsschwere und dem Pflegeaufwand assoziiert. Es wurden Kosten pro TISS - Punkt von 35 Euro (Kostenzeitpunkt 1995) (Lefering et al. 1997) bzw. 36 Euro (Kostenzeitpunkt 1998) (Graf et al. 2002) ermittelt. Eine weitere Studie von Kaufmann und Briegel ergab einen Wert von 38 Euro pro TISS - Punkt (inklusive Personalkosten und Overhead, Kostenzeitpunkt 1998) (Kaufmann und Briegel 2000).

Auch in der vorliegenden Untersuchung konnte eine Korrelation zwischen Gesamt - TISS und den Gesamtkosten gezeigt werden. Der 
Korrelationskoeffizient beträgt hier 0,75 und die errechneten Kosten pro TISS - Punkt liegen mit 45 Euro geringfügig höher als die von den obigen Autoren ermittelten Werte.

Jedoch ist in Anbetracht der verschiedenen TISS - Versionen ein Vergleich der Kosten pro TISS - Punkt aus verschiedenen Publikationen schwierig, da die Kosten pro TISS - Punkt abhängig von der eingesetzten TISS - Version sind. So benutzten Dickie et al. z. B. noch den TISS in der alten Version, während Graf et al. den TISS - 28 verwendeten. Dickie et al. haben die realen ICU - Kosten mit den per TISS - Wert geschätzten Kosten verglichen. Der Unterschied lag im Durchschnitt bei 7,7\%. Dies impliziert, dass der TISS sogar als zuverlässiger Aufwandmarker im ICU - Management genutzt werden könnte (Dickie et al. 1998).

Da sich der TISS bereits als guter Indikator des intensivmedizinischen Pflegeaufwandes erwiesen hat, ist es nahe liegend, dass auch die Kosten mit dem Score gut korrelieren, da sie proportional zum Pflegeaufwand steigen.

Zusammenfassend kann man sagen, dass in mehreren Studien der TISS als ein verlässlicher Marker der Kosten für Intensivpatienten bewertet wurde und somit eine wichtige Rolle im ICU - Kostenmanagement spielen könnte. Der TISS wird bereits in einigen Intensivstationen als eine patientenbezogene Kalkulationsmethode für Ressourcen genutzt. In der Studie von Dickie et al. wurde dessen Anwendbarkeit validiert.

Bei der Suche nach einem zuverlässigen Aufwandmarker für die intensivmedizinischen Leistungen im neuen deutschen DRG- System hat der TISS jedoch den Nachteil, dass er durch die Behandler selbst beeinflusst werden kann. Diese bestimmen den angewandten Aufwand in der Behandlung. Somit wäre der TISS als Aufwandmarker nicht unabhängig von einer möglichen Manipulation durch die Behandler. Infolgedessen fand der TISS nicht das Vertrauen seitens der politischen Entscheidungsträger. 
In der Studie von Graf et al. korrelierte der einmalig, am Beginn der Intensivbehandlung bestimmte pathophysiologische Score SAPS II nicht mit dem Ressourcenverbrauch (Graf et al. 2002).

Demgegenüber wurde in der vorliegenden Arbeit ein anderes Konzept eingesetzt: Mit den aufsummierten täglich gemessenen SAPS II - Werten (Gesamt - SAPS II) korrelieren jetzt die Gesamtkosten mit einem Korrelationskoeffizienten von 0,75. Es ist verständlich, dass die Ausgaben für Therapie und Diagnostik mit der durch den Score quantifizierten Krankheitsschwere korrelieren. Wichtiger jedoch ist, dass durch Aufsummierung der SAPS - Werte über die gesamte Verweildauer auf der Intensivstation die LOS stark berücksichtigt wird. Damit gehen auch die täglich anfallenden Fixkosten, wie insbesondere die Personalkosten, als gewichtiger Kostensockel in die Berechnung ein. Wie man in der Darstellung der Verteilung der einzelnen Kostenblöcke anhand der Musterpatienten erkennen kann, bilden die Personalkosten täglich den Hauptteil der Gesamtkosten. Wenn man bedenkt, dass in unserer Studie allein die Personalkosten bereits $57,8 \%$ betragen, dann ist die gute Korrelation nicht verwunderlich.

So stellten auch Moerer und seine Mitarbeiter für den Gesamt - SAPS (d.h. den Schweregradscore SAPS, täglich bestimmt und für die gesamte Liegedauer aufsummiert) eine bemerkenswert gute Korrelation zum Kostenaufwand fest. Damit wäre der Gesamt - SAPS ein sachgerechter Aufwandsmarker für die Vergütung der Intensivmedizin. Er berücksichtigt einerseits die LOS mit dem hohen Fixkostenanteil durch Personal, andererseits liefert er mit der Höhe des täglichen SAPS aber auch eine Begründung der intensivmedizinischen Behandlung.

Eine an den Krankheitsschwerescore SAPS II angelehnte Vergütung könnte jedoch mit einigen Problemen einhergehen, da der Score die Glasgow Coma Scale (GCS) beinhaltet. Diese ist schwierig zu evaluieren und kann bei sedierten Patienten überhaupt nicht zur neurologischen Beurteilung herangezogen werden. 
Des Weiteren müssten zur täglichen Scoreerhebung alle, zum Teil sehr kostenintensiven, Laborparameter täglich bestimmt werden. Da aber die täglichen Laborparameterbestimmungen klinisch nicht immer indiziert sind, wäre dies nicht nur mit weiteren Umständen für den Patienten verbunden, was unter ethischen Gesichtspunkten inakzeptabel wäre, sondern auch mit weiteren und zudem noch unnötigen Kosten.

Zur Lösung dieses Problems wurde im DRG - System die Regelung eingeführt, dass der einmal ermittelter Laborwert solange täglich unverändert wieder in die Berechnung des Gesamtscores einfließt, bis er das nächste Mal neu erhoben wird. In der vorliegenden Studie wurde so verfahren. Der Nachteil dieser Regelung könnte sein, dass der Krankheitsverlauf nicht immer zeitgenau evaluiert wird. Andererseits kann aber angenommen werden, dass bei klinischen Veränderungen rasch erneute Laborkontrollen veranlasst werden. Mit der Zunahme der Untersuchungsfrequenz ist die Wahrscheinlichkeit, pathologische Werte zu erfassen, naturgemäß höher.

Eine an den TISS - 28 angelehnte Vergütung würde sich in erster Linie nach den Tätigkeiten des medizinischen Personals richten. Eine standardisierte und zuverlässige Erfassung aller getätigten Prozeduren erscheint mit Hilfe dieses Scores möglich (Moreno 2001). Auch andere Studien deuten darauf hin, dass sich die Patientenkosten, die im direkten Zusammenhang mit den pflegerischen Tätigkeiten stehen, gut vom TISS ableiten lassen (Slatyer et al. 1986, Dickie et al. 1998). Ferner bleibt im TISS auch die Krankheitsschwere indirekt berücksichtigt, da der pflegerische Aufwand fast immer mit einer Verschlechterung des Krankheitszustandes steigt. Da die Scoreerhebung standardisiert und im Vergleich zu anderen Scores relativ einfach ist, könnte die Errechnung der individuellen Patientenkosten mit Hilfe des TISS - 28 schnell erfolgen (Graf et al. 2002).

Der TISS beschreibt also den geleisteten Aufwand durchaus nachvollziehbar (Graf et al. 2002), der Behandelnde bestimmt aber den Behandlungsaufwand selbst und entscheidet damit über die Höhe des 
TISS - Wertes. Dabei sei aber nochmals betont: Ein Aufwandsmarker zur Vergütung, der ausschließlich auf Behandlungsaktionen basiert, welche die Behandler selbst bestimmen können - der also (willkürlich?) beeinflussbar gewesen wäre - hätte bei den politischen Entscheidungsträgern keine Akzeptanz gefunden.

Nachteilig beim TISS - System ist auch, dass es sich um ein inzwischen relativ betagtes System handelt. Therapeutische Möglichkeiten, die vor vielen Jahren noch keine Rolle gespielt haben mögen, werden nicht oder nur unzureichend berücksichtigt (Miranda et al. 1996).

Obwohl Scoresysteme wie SAPS und TISS bereits seit Jahren angewendet werden, gibt es im Einzelfall gelegentlich Unklarheiten und Anwendungsprobleme, mit der Folge, dass die in den jeweiligen Originalarbeiten vorgegebene Verfahrensweise nicht immer einheitlich übernommen wird. Auch das Vorgehen bei Wiederaufnahme auf die Intensivstation ist nicht in allen Details festgelegt. Des Weiteren lassen die unterschiedlich genau definierten Ausschlusskriterien Spielraum in der Anwendung der Scoresysteme zu (Unertl und Kottler 1997).

Auch in der vorliegenden Studie werden die Scoresysteme inrem Ursprungssinn zweckentfremdet, indem sie mit den intensivmedizinischen Kosten korreliert werden.

Die Schweregradscores in der Intensivmedizin dienen zur Prognose der Krankheitsmortalität im Moment des Aufnahmezeitpunkts. Zur Berechnung dieser Scores werden ausschließlich Werte der ersten 24 Stunden herangezogen, in denen die Auswirkung therapeutischer Maßnahmen als gering zu erachten ist.

Janssens et al. stützen diese Überlegung durch eine Studie, in der sie SAPS II, TISS - 28 und APACHE II miteinander vergleichen. Da es gerade unter dem Einfluss therapeutischer Maßnahmen zu einer Stabilisierung vieler physiologischer Funktionen kommt bzw. diese künstlich verbessert werden, nehmen auch bei versterbenden Patienten die Scorewerte im Verlauf ab (Janssens et al. 2000). Das klinische Profil eines Patienten 
ändert sich also in Reaktion auf seine Therapie. Korrekterweise müssten für die Prognose daher Scores verwendet werden, in die die therapeutischen Maßnahmen mit eingerechnet werden.

Zudem wurde der SAPS II wurde als Aufnahmescore entwickelt und ist nicht als Verlaufsscore validiert (Weiss et al. 2005). So ist die Verwendung des SAPS II - Scores zur Aufwandsdokumentation aus mehreren Gründen kritisch zu werten.

Es wäre dennoch notwendig, den Krankheitsschweregrad regelmäßig zu erfassen, um eine akkurate Abschätzung der Prognose zu erhalten. Jedoch kann der Verlauf nicht einfach durch die wiederholte bzw. tägliche Anwendung etablierter Scoringsysteme, die als Aufnahmescores entwickelt und validiert wurden, abgebildet werden. Offensichtlich sind hier andere Parameter, eine andere Gewichtung oder die Kombination von Zeitpunkten notwendig, um eine adäquate Aussage über die Änderung des Patientenzustands treffen zu können.

Der erst kürzlich entwickelte SAPS III (Metnitz et al. 2005, Moreno et al. 2005) zur Einschätzung von Krankheitsschwere und Mortalität kann eingeschränkt zu den Verlaufsscores für die tägliche Anwendung gezählt werden, da er zur Erhebung an den Aufenthaltstagen $1-3$ und dem Entlassungstag validiert wurde (Brenck et al. 2008). Hingegen zeigte sich der in der vorliegenden Arbeit angewendete SAPS II in der täglichen Verwendung als ungeeignet zur Prognosenabschätzung (Sicignano et al. 1996).

Für alle Scoresysteme gilt nicht zuletzt, dass auch die Untersuchervariabilität zu berücksichtigen ist.

In der vorliegenden Studie wurde für das gesamte Patientengut eine Korrelation zwischen Patientengesamtkosten und dem TISS - 28 gezeigt. Auch für die Autoren Dickie, Slatyer und Graf besitzt der TISS eine hohe ökonomische Aussagekraft. So sollte in Anbetracht dieser Studien, aber auch der eigenen Untersuchungen gegenwärtig bei der 
Vergütungsbemessung eigentlich dem TISS - 28 der Vorrang eingeräumt werden.

Allerdings weist in den Ergebnissen dieser Studie auch der SAPS II eine positive Korrelation mit den Gesamtkosten auf und ist daher als Vergütungsparameter, trotz der bereits genannten Schwierigkeiten bei seiner Erhebung, denkbar.

Auf der Grundlage der Vorgaben wurde durch die politischen Entscheidungsgremien daher als Aufwandmarker für die Vergütung intensivmedizinischer Leistungen (sog. „intensivmedizinische Komplexbehandlung") seinerzeit der auf die Dauer der intensivmedizinischen Behandlung aufsummierte SAPS II (ohne GCS) sowie zusätzlich einige gewichtige TISS - 28 - Werte (sog. "Core - 10 - TISS“) gewählt. Damit wird die wegen der Personalkosten wichtige LOS ebenso wie der Schweregrad der Erkrankung gebührend berücksichtigt.

Es ist verständlich, dass ein Fallpauschalensystem nicht jeden Patienten mit seiner spezifischen Diagnostik und Therapie individuell komplett und korrekt abbilden und vergüten kann. Innerhalb einer G - DRG - Fallgruppe erfolgt eine Vergütung im Mittel, bei der kostenintensivere Fälle durch Fälle mit geringeren Kosten kompensiert werden müssen.

\subsection{Ausblick}

Unabhängig von neuen Modellen zur Vergütungsbemessung müssen auch weiterhin Einsparmaßnahmen erfolgen, wie beispielsweise das Vermeiden unnötiger Doppeluntersuchungen oder die weitere Optimierung der Pharmakotherapie. Insbesondere sollte die Verkürzung der LOS oberstes Ziel der Einsparmaßnahmen sein, da die Höhe der Gesamtkosten in erster Linie mit der intensivstationären LOS verbunden ist (Neilson et al. 2004). 
Eine Studie von Blackstone und seinen Mitarbeitern zeigt, dass das Kosteneinsparungspotential durch eine Ressourcenoptimierung seitens der Mitarbeiter mit $28 \%$ bei Medikamentenausgaben, $21 \%$ bei Laborleistungen und $29 \%$ bei Röntgen - Thoraxaufnahmen als relativ hoch einzuschätzen ist, ohne dass die Letalität beeinflusst wird (Blackstone et al. 1995).

Bezogen auf die heutige Situation im Gesundheitswesen können diese Angaben allerdings nicht als ein allgemein geltender Maßstab des vorhandenen Einsparungspotentials dienen. Denn es haben aufgrund der seit Jahren angespannten Finanzlage im Gesundheitssystem bereits große, teils erfolgreiche Anstrengungen zur Kostensenkung stattgefunden. Angesichts hoher und stetig steigender Kosten und begrenzter Ressourcen werden Leistungen immer stärker auch unter dem Gesichtspunkt der Kosteneffektivität und Qualität beurteilt werden müssen.

Um im schärfer werdenden Wettbewerb um immer knapper werdende Ressourcen bestehen zu können, müssen wir neben der Entwicklung von neuen kostenträchtigen Therapieformen und Technologien auch die bestehenden Behandlungsprozesse und Strukturen optimieren, d. h., das erwünschte Ziel mit dem geringstmöglichen Aufwand zu erreichen. Hierin wird sichtbar, dass Qualität künftig nicht mehr nur allein an der Effektivität (Outcome) gemessen werden wird, sondern auch die Effizienz Berücksichtigung findet.

Hierbei lassen sich der Therapieaufwand im Verhältnis zur Krankheitsschwere und der LOS als wichtige Indikatoren einer optimalen Ressourcennutzung heranziehen. So wird es durch Verknüpfung verschiedener Scoresysteme, wie beispielsweise SAPS II und TISS - 28, möglich, den Aufwand im Verhältnis zur Krankheitsschwere darzustellen und einer Effizienzanalyse zu unterziehen (Abizanda und Rodriguez 1996). 


\section{Zusammenfassung}

Im Rahmen des GKV Gesundheitsreformgesetzes 2000 vom 22.12.1999 wurde die Einführung eines durchgängig leistungsorientierten und pauschalierenden Entgeltsystems für die Vergütung von Krankenhausleistungen im $\S 17 \mathrm{~b}$ des Krankenhausfinanzierungsgesetzes geregelt. Die Umstellung auf das G - DRG - System beendete die Vergütung nach Tagessätzen. Seither sind die Entgelte für Patientenbehandlungen unabhängig vom individuellen Krankheitsverlauf und dem damit verbundenen Behandlungsaufwand. Mit der Einführung des pauschalierten G - DRG - Systems als Grundlage der Vergütung klinischer Leistungen ist die intensivmedizinische Kostendeckung problematisch geworden.

Eine Vergütung auf der Grundlage der Scores SAPS II und TISS - 28 wäre eine Möglichkeit zur Deckung der durch intensivmedizinische Patienten verursachten Kosten. Der SAPS II ist ein Krankheitsschwerescore, währenddessen der TISS - 28 der Erfassung des Leistungsaufwandes dient.

Gegenstand dieser Studie war die Prüfung einer möglichen Korrelation zwischen diesen Scores und den direkten patientenbezogenen Kosten, welche durch die intensivmedizinische Behandlung anfielen. Diese Analyse war Teil einer multizentrischen, nationalen Kostenerfassungsstudie, die als Entscheidungshilfe für eine kostengerechte Vergütung der Intensivbehandlung dienen sollte. Abhängig von deren Ergebnissen sollte letztlich eine Aussage getroffen werden, ob ein an Scores angelehntes Vergütungsmodell für den Leistungserbringer eine Kostendeckung der individuellen Patientenkosten garantieren kann.

In die statistische Auswertung dieser Studie, welche im Zeitraum von Juni bis Oktober 2004 durchgeführt wurde, gingen die Daten von 100 Patienten der internistischen Intensivstation 1026 des Universitätsklinikums Göttingen ein. 
Dabei konnte eine positive Korrelation sowohl zwischen den täglich über die intensivmedizinische Verweildauer aufsummierten Werten des SAPS II und des TISS - 28 einerseits und den Gesamtkosten pro Patient andererseits nachgewiesen werden. Der Korrelationskoeffizient beträgt für den Gesamt-SAPS-II-Score 0,73 und für den TISS-28-Leistungsscore 0,75 .

Einschränkend muss jedoch erwähnt werden, dass auf Tagesbasis keine Korrelation zwischen den in dieser Arbeit untersuchten Scores und den Kosten besteht. Doch das ist für eine Pauschal - Vergütung auch nicht unbedingt erforderlich.

Die Vergütung der intensivmedizinischen Kosten auf der Grundlage eines Aufwandmarkers, der auf den täglich aufsummierten Scorewerten beruht, scheint nach den Ergebnissen der vorliegenden Studie durchaus möglich. Auf diese Weise würden sich die Entgelte in erster Linie nach dem individuellen Krankheitsverlauf und dem daraus resultierenden Therapie und Pflegeaufwand eines Patienten richten. 


\section{Abkürzungsverzeichnis}

\begin{tabular}{|c|c|c|}
\hline AMI & $=$ & $\begin{array}{l}\text { Akuter Myokardinfarkt } \\
\text { (Acute Myocardial Infarction) }\end{array}$ \\
\hline APACHE & $=$ & $\begin{array}{l}\text { Krankheitsschwerescore } \\
\text { (Acute Physiology And Chronic Health Evaluation) }\end{array}$ \\
\hline BiPAP & $=$ & $\begin{array}{l}\text { Respirationsmodus } \\
\text { (Biphasic Positive Airway Pressure) }\end{array}$ \\
\hline CPAP & $=$ & $\begin{array}{l}\text { Spontanatmung mit positiven endexspiratorischem } \\
\text { Druck } \\
\text { (Continuous Positive Airway Pressure) }\end{array}$ \\
\hline COPD & $=$ & $\begin{array}{l}\text { Chronisch obstruktive, pulmonale Erkrankung } \\
\text { (Chronic Obstructive Pulmonal Disease) }\end{array}$ \\
\hline CT & $=$ & $\begin{array}{l}\text { Röntgenschichtuntersuchung } \\
\text { (Computertomographie) }\end{array}$ \\
\hline DIVI & $=$ & $\begin{array}{l}\text { Deutsche Interdisziplinäre Vereinigung für } \\
\text { Intensivmedizin und Notfallmedizin }\end{array}$ \\
\hline DRG & $=$ & $\begin{array}{l}\text { Leistungsvergütungssystem nach Fallpauschalen } \\
\text { (Diagnosis - Related - Group) }\end{array}$ \\
\hline EKG & $=$ & Elektrokardiographie \\
\hline GCS & $=$ & $\begin{array}{l}\text { Bewusstseinsbewertungsscala } \\
\text { (Glasgow Coma Scale) }\end{array}$ \\
\hline IABP & $=$ & Intraaortale Ballonpumpe \\
\hline$I C D-10$ & $=$ & $\begin{array}{l}\text { Internationale Klassifikation der Krankheiten } \\
\text { (International Classification of Disease - 10) }\end{array}$ \\
\hline ICU & $=$ & $\begin{array}{l}\text { Intensivstation } \\
\text { (Intensive Care Unit) }\end{array}$ \\
\hline IMC & $=$ & $\begin{array}{l}\text { Überwachungsstation } \\
\text { (Intermediate Care) }\end{array}$ \\
\hline InEK & $=$ & Institut für das Entgeltsystem im Krankenhaus \\
\hline LMV & $=$ & $\begin{array}{l}\text { Beatmungsdauer } \\
\text { (Length of Mechanical Ventilation) }\end{array}$ \\
\hline LOS & $=$ & $\begin{array}{l}\text { Liegedauer } \\
\text { (Length of Stay) }\end{array}$ \\
\hline
\end{tabular}




\begin{tabular}{|c|c|c|}
\hline MRSA & $=$ & Multiresistenter Staphylococcus aureus \\
\hline OPS & $=$ & Operationen - und Prozedurenschlüssel \\
\hline PEG & $=$ & $\begin{array}{l}\text { Magenernährungssonde } \\
\text { (Perkutane, endoskopische Gastrostomie) }\end{array}$ \\
\hline PTCA & $=$ & $\begin{array}{l}\text { Herzkatheteruntersuchung mit Stenteinlage } \\
\text { (Perkutane, transluminale koronare Angioplastie) }\end{array}$ \\
\hline SAPS & $=$ & $\begin{array}{l}\text { Krankheitsschwerescore } \\
\text { (Simplified Acute Physiology Score) }\end{array}$ \\
\hline SIMV & $=$ & $\begin{array}{l}\text { Kombination aus assistierter Spontanatmung und } \\
\text { Kontrollierten Beatmungshüben } \\
\text { (Synchronized Intermittend Mandatory Ventilation) }\end{array}$ \\
\hline TEE & $=$ & Transösophageale Eckokardiographie \\
\hline TISS & $=$ & $\begin{array}{l}\text { Pflegeaufwandsscore } \\
\text { (Therapeutic Intervention Scoring System) }\end{array}$ \\
\hline VUD & $=$ & Verband der Universitätsklinika Deutschlands \\
\hline
\end{tabular}




\section{Literaturverzeichnis}

Abizanda R, Rodriguez MT (1996): The purpose and use of prognostic indices in critical care medicine. Curr Opin Crit Care 2, 214-220

Angus DC, American Thoracic Society (2002): Understanding cost and cost - effectiveness in critical care: Report from the second American Thoracic Society Workshop on outcomes research. Am J Respir Crit Care Med 165(4), 540 - 550

Apgar $V$ (1953): A proposal for a new method of evaluation of the newborn infant. Curr Res Anaesth Analg 32(4), 260 - 267

Apolone G, Bertolini G, D’Amico R, lapichino G, Cattaneo A, De Salvo G, Melotti RM (1996): The performance of the SAPS II in a cohort of patients admitted to 99 Italian ICUs: results from GiViTI. Gruppo Italiano per la Valutazione degli interventi in Terapia Intensiva. Intensive Care Med $\underline{\text { 22(12), }}, 1668-1678$

Billing $A$, Thalhammer $M$, Eissner $H J$, Jauch KW, Inthorn D (2004): Economic Aspects of Intensive Care Medicine - Cost and Reimbursement according to Diagnosis related Grouping. Zentralbl Chir $\underline{129}, 440$ - 446

Blackstone ME, Miller RS, Hodgson H (1995): Lowering hospital charge in the trauma intensive care unit while maintaining quality of care by increasing resident and attending physician awareness. J Trauma $\underline{39}$, $1041-1044$

Brenck F, Hartmann B, Mogk M, Junger A (2008): Intensivmedizinische Scoringsysteme zur täglichen Anwendung: Übersicht, aktuelle Möglichkeiten und Anforderungen an Neuentwicklungen. Anaesthesist $\underline{57}$, $189-195$ 
Burchardi H (2004): DRG und Intensivmedizin: Bedrohung oder Chance? Intensiv- und Notfallbehandlung 29(4), 198 - 209

Chaix C, Durand- Zeleski I, Albertini C, Brun- Buisson C (1999): A Model to Compute the Medical Cost of Patients in Intensive Care. Pharmacoeconomics 15(6), $573-582$

Cullen DJ (1977): Results and costs of intensive care. Aneasthesiology $\underline{47}, 203-216$

Cullen DJ, Civetta JM, Briggs BA, Ferara LC (1974): Therapeutic Intervention Scoring System: a method of quantitative comparison of patient care. Crit Care Med 2, $57-60$

Deutsches Institut für medizinische Dokumentation und Information: ICD - 10: Internationale statistische Klassifikation der Krankheiten und verwandter Gesundheitsprobleme. Deutscher Ärzte - Verlag, Köln 1995

Dickie H, Vedio A, Dundas R, Treacher DF, Leach RM (1998): Relation ship between TISS and ICU cost. Intensive Care Med 24, 1009 - 1017

Duckett SI (1998): Casemix funding for acute hospital inpatient services in Australia. Med J Austr 169, 17 -21

Edbrooke DL, Hibbert CL, Ridley S, Long T, Dickie H (1999): The development of a method for comprative costing of individual intensive care units. The Intensive Care Working Group on Costing. Anaesthesia $\underline{54(2)}, 110-129$

Ferreira FL, Bota DP, Bross A, Melot C, Vincent JL (2001): Serial evaluation of the SOFA score to predict outcome in critically ill patients. JAMA 286(14), $1754-1758$ 
Graf J, Graf C, Janssens U (2002): Analysis of resource use and cost generating factors in a german medical intensive care unit employing the Therapeutic Intervention Scoring System (TISS - 28). Intensive Care Med $\underline{28(3)}, 324-331$

Gyldmark M (1995): A review of cost studies in intensive care units: problems with the cost concept. Crit Care Med $\underline{23}, 964-972$

Havill JH, Sleigh JW, Kersel DA, Marsh NV (1998): Profile and cost of head injury patients admitted to the Waikato Hospital Intensive Care Unit. N Z Med J 111(1065), 161 - 163

Heyland DK, Konopad E, Noseworthy TW, JohnstonR, Gafni A (1998): Is it "worthwile" to continue treating patients with a prolonged stay (> 14 days) in the ICU? An economic evaluation. Chest 114(1), 192 - 198

lapichino $G$, Radrizzani D, Simini B, Rossi C, Albicini M, Ferla L, Colombo A, Pezzi A, Brazzi L, Melotti R, Rossi G (2004): Effectiveness and efficiency of intensive care medizine: variable costs in different diagnosis groups. Acta Anaesthesiol Scand 48(7), 820 - 826

Janssens U, Graf C, Graf J, Radke PW, Königs B, Koch KC, Lepper W, vom Dahl J, Hanrath P (2000): Evaluation of the SOFA score: a single - center experience of a medical intensive care unit in 303 consecutive patients with predominantly cardiovascular disorders. Intensive Care Med 26, 1037 - 1045

Jegers $M$, Edbrooke DH, Hibbert CL, Chalfin DB, Burchardi H (2002): Definitions and methods of cost assessment: an intensivist's guide. ESICM Section on health services research and outcome working group on cost effectiveness. Intensive Care Med 28(6), 680 - 685 
Kaufmann I, Briegel J (2000): Therapeutic Intervention Scoring System (TISS) - a method for calculating costs in the intensive care unit (ICU) and intermediate care unit (IMCU). Crit Care 4(1), S243

Klepzig $\mathrm{H}$, Winten $\mathrm{G}$, Thierolf $\mathrm{C}$, Kiesling G, Usadel KH, Zeiher AM (1998): Treatment costs in a medical intensive care unit: a comparison of 1992 and 1997. Dtsch Med Wochenschr 123(23), 719 - 725

Knaus WA, Draper EA, Wagner DP, Zimmermann JE (1985): APACHE II: a severity of disease classification system. Crit Care Med $\underline{13}, 818-829$

Lefering $R$, Zart $M$, Neugebauer $E$ (1997): Effektivkosten der Intensivtherapie: TISS und TISS -28 zur Evaluation einer Intensivstation. Langenbecks Arch Chir 114, 1393 - 1395

Le Gall JR, Lemeshow S, Saulnier F (1993): A new Simplified Acute Physiology Score (SAPS II) basaed on a European / North American multicenter study. JAMA 270(24), 2957 - 2963

Martin J, Neurohr C, Bauer M, Weiß M, Schleppers A (2008): Cost of intensive care in a German Hospital: Cost - unit accounting based on the InEK matrix. Anaesthesist 57(5), $505-512$

Metnitz PGH, Valentin A, Vesely H, Albertini C, Lang K, Steltzer H, Hiesmayr (1999): Prognostic performance and customization of the SAPS II: results of a multicenter Austrian study. Intensive Care Med $\underline{25}$, $192-197$

Metnitz PGH, Moreno RP, Almeida E (2005): SAPS 3 - From evaluation of the patient to evaluation of the intensive care unit. Part 1: objectives, methods and cohort description. Intensive Care Med 31, 1336 - 1344 
Miranda DR, Rijk A, Schaufeli W (1996): Simplified Therapeutic Intervention Scoring System: The TISS - 28 items - Results from a multicenter study. Crit Care Med 24(1), $64-73$

Moerer O, Burchardi $H$ (2001): Cost profiles of direct costs in ICU patients. Intensive Care Med 27, S208

Moerer O, Schmid A, Hoffmann M, Herklotz A, Reinhart K, Werdan K, Schneider H, Burchardi H (2002): Direct costs of severe sepsis in three German intensive care units based on retrospective electronic patient record analysis of resource use. Intensive Care Med 28(10), 1440 - 1446

Moerer O, Plock E, Mgbor U, Schmid A, Schneider H, Wischnewky MB, Burchardi H (2007): A German national prevalence study on the cost of intensive care: an evaluation from 51 intensive care units. Crit Care 11(3), R69

Moreno R: Severity of illness: Evaluating critical care; hrsg. v. Sibbald WJ, Bion JF. Springer - Verlag, Berlin 2001, 51-68

Moreno R, Morais P (1997): Outcome prediction in intensive care: results of a prospective, multicentre, Portuguese study. Intensive Care Med $\underline{23}$, $177-186$

Moreno RP, Metnitz PGH, Almeida E (2005): From evaluation of the patient to evaluation of the intensive care unit. Part 2: development of a prognostic model for hospital mortality at ICU admission. Intensive Care Med $\underline{31}, 1345-1355$

Neilson AR, Moerer O, Burchardi H, Schneider H (2004): A new concept for DRG - based reimbursement of services in German intensive care units: results of a pilot study. Intensive Care Med $\underline{30(6)}, 1220$ - 1223 
Noseworthy TW, Konopa E, Shustack A, Johnston R, Grace M (1996): Cost accounting of adult itensive care: methods and human and capital inputs. Crit Care Med 24(7), 1168 - 1172

Parviainen I, Herranen A, Holm A, Uusaro A, Ruokonen E (2004): Results and costs of intensive care in a tertiary university hospital from $1996-$ 2000. Acta Anaesthesiol Scand $\underline{48}, 55-60$

Reina A, Vazquez G, Aguayo E, Bravo I, Colmenero M, Bravo M, PAEEC Group (1996): Mortality discrimination in acute myocardial infarction: comparison between APACHE III and SAPS II prognosis systems. Intensive Care Med $\underline{23}, 326-330$

Reinecke $\mathrm{H}$, Bunzemeier $\mathrm{H}$, Fürstenberg $\mathrm{T}$, Rothenburger $\mathrm{M}$, Böcker $\mathrm{D}$, Scheld HH, Breithardt G, Roeder N (2003): Evaluating the first German diagnosis - related groups (G - DRG) in cardiological patients: problems in the correct medical and economic grouping. Z Kardiol 92(7), $581-594$

Schmidt A, Moerer O, Neilson A, Burchardi H, Schneider H (2004): The introduction of new therapies in intensive care: clinical advantages at additional cost. Gesundh Oekon Qual Manag 9(2), 93 - 96

Schuster HP, Wilts S, Ritschl P, Schuster FP (1996): Predictive value of score parameteres of the simplified Acute Physiology Score (SAPS)-II fort he duration of treatment of intensive care patients. Wien Klin Wochenschr $\underline{108(15)}, 462-466$

Schuster HP, Schuster FP, Ritschel P, Wilts S, Bodmann KF (1997): The ability of the Simplified Acute Physiology Score (SAPS II) to predict outcome in coronary care patients. Intensive Care Med $\underline{23}, 1056$ - 1061

Sicignano A, Carozzi C, Giudici D (1996): The influence of length of stay in the ICU on power of discrimination of a multipurpose severity score (SAPS). Intensive Care Med 22, 1048 - 1051 
Slatyer MA, James O, Moore PG, Leeder SR (1986): Costs, severity of illness and outcome in intensive care. Anaesth Intensive Care 14, $381-389$

Staudacher J, Moerer O, Burchardi $\mathrm{H}$ (2003): Cost refunding for intensive care in a DRG - System. Intensive Care Med 27, 208

Stiletto RJ, Schäfer E, Waydhas C (2000): Qualitätssicherung in deutschen Intensivstationen: erste Ergebnisse einer prospektiven Querschnittsstudie der Interdisziplinären Arbeitsgemeinschaft Qualitätssicherung in der Intensivmedizin der DIVI. Intensivmedizin $\underline{37}$, $608-616$

Tarnow - Mordi WO, Hau C, Warden A, Shearer AJ (2000): Hospital mortality in relation to staff workload: a 4 - year study in an adult intensive - care unit. Lancet $\underline{356}, 185$ - 189

Unertl K, Kottler BM (1997): Prognostische Scores in der Intensivmedizin. Anaesthesist $\underline{46}, 471-480$

Van de Werf F, Ardssino D, Betriu A (2003): Management of acute myocardial infarction in patients presenting with ST - segment elevation. Eur Heart J $\underline{24}, 28-66$

Vosylius S, Sipylaite J, Ivaskevicus J (2004): Evaluation of intensive care unit performance in Lithuania using the SAPS II system. Eur J Anaesthesiol 21(8), $619-124$

Weiss M, Martin J, Feser , Schafmayer C, Bauer M (2005): Notwendigkeit und Probleme einer fallbezogenen Kostenträgerrechnung bei intensivtherapiepflichtigen Patienten. Anaesthesist $\underline{54}, 254-262$ 


\section{Danksagung}

Zuerst möchte ich mich bei Herrn Prof. Dr. med. H. Burchardi für die Überlassung des Themas dieser Dissertation, die sehr gute Betreuung und die große Unterstützung bei dieser Arbeit herzlich bedanken.

Des Weiteren danke ich auch Herrn PD Dr. med. O. Mörer für die nette Betreuung. Wenn es Probleme oder Fragen gab, konnte man sowohl den Doktorvater als auch den Betreuer jederzeit konsultieren. Besonders in der Phase der Datenerhebung, bei wissenschaftlichen Problemen und beim Korrigieren der Dissertation waren sie mir eine große Hilfe.

Für das Ermöglichen dieser Dissertation durch die Zusammenarbeit mit der Abteilung für Kardiologie und Pneumologie im Zentrum Innere Medizin möchte ich mich ausdrücklich bei Herrn Prof. Dr. med. G. Hasenfuß bedanken. Auch bedanke ich mich bei dem gesamten Team der Intensivstation 1026 für das freundliche Klima und die Unterstützung bei der Dokumentation der Patientendaten.

Für die statistische Datenauswertung danke ich der Fa. Health Econ AG in Basel. Mein ganz persönlicher Dank gilt dabei Frau Dr. E. Brock.

Weiterhin möchte ich mich bei der Abteilung für Medizinische Statistik der Universität Göttingen für die nette studentische Betreuung im CIP Raum und die Unterstützung bei der statistischen Auswertung meiner Daten sowie der Erstellung der Diagramme, Box Plots und Scatterplots bedanken.

Des Weiteren bedanke ich mich beim Dissertationsbeauftragten der Medizinischen Fakultät der Universität Göttingen, Herrn Dr. K. H. Stubenrauch, für das Überprüfen meiner Dissertationsarbeit gemäß den Richtlinien der Medizinischen Fakultät. 